Interfaces and Free Boundaries 14 (2013), 1-37

DOI 10.4171/IFB/293

\title{
Adhesive contact delaminating at mixed mode, its thermodynamics and analysis
}

\author{
RICCARDA ROSSI \\ Dipartimento di Matematica, Università di Brescia, Via Valotti 9, I-25133 Brescia, Italy \\ E-mail: riccarda.rossi@ing.unibs.it \\ TOMÁš ROUBÍČEK \\ Mathematical Institute, Charles University, Sokolovská 83, CZ-18675 Praha 8, and \\ Institute of Thermomechanics of the ASCR, Dolejškova 5, CZ-18200 Praha 8, Czech Republic \\ E-mail: tomas.roubicek@mff.cuni.cz
}

[Received 10 October 2011 and in revised form 21 July 2012]

\begin{abstract}
An adhesive unilateral contact problem between visco-elastic heat-conductive bodies in linear Kelvin-Voigt rheology is scrutinized. The flow-rule for debonding the adhesive is considered rate-independent, unidirectional, and non-associative due to dependence on the mixity of modes of delamination, namely of Mode I (opening) and of Mode II (shearing). Such mode-mixity dependence of delamination is a very pronounced (and experimentally confirmed) phenomenon typically considered in engineering models. An anisothermal, thermodynamically consistent model is derived, considering a heat-conductive viscoelastic material and the coupling via thermal expansion and adhesion-depending heat transition through the contact surface. We prove the existence of weak solutions by passing to the limit in a carefully designed semi-implicit time-discretization scheme.
\end{abstract}

2010 Mathematics Subject Classification: Primary 35K85; Secondary 35Q79, 74A15, 74A30, 74R20, 80A17.

Keywords: Contact mechanics; rate-independent processes; heat equation; energetic solution.

\section{Introduction}

Nonlinear contact mechanics is an important part of mechanical engineering and receives still growing attention due to its numerous applications. We focus on the modelling and analysis of an inelastic process called delamination (sometimes also referred to as debonding), between two elastic bodies, glued together along a prescribed delamination surface. "Microscopically" speaking, some macromolecules in the adhesive may break upon loading and we assume that they can never be glued back, i.e., no "healing" is possible. This makes the process unidirectional, viz. irreversible. On the glued surface, we consider the delamination process as rate-independent and, in the bulk, we also take into account rate-dependent inertial, viscous, and heat-transfer effects. The ultimate phenomenon counted in engineering modelling (and so far mostly ignored in the mathematical literature), is the dependence of this process on the modes under which it proceeds. Indeed, Mode I (=opening) usually dissipates much less energy than Mode II (=shearing). The difference may be tens or even hundreds of percents, cf. [1, 22, 23, 47]. Moreover, the delamination process rarely follows such pure modes: in general, the mixed mode is favored. Microscopically, this difference is explained either by some roughness of the glued surface (to be overcome in Mode II but not in Mode I, cf. [11]) or by some plastification, either in the adhesive or in a narrow strip around the 
delaminating surface, before the delamination itself is triggered (and, as usual in plasticity involving trace-free plastic strain, again only shearing in Mode II can trigger this plastification, not opening in Mode I, cf. [22, 51]).

In this article, we focus on a standard engineering model which, however, was never rigorously analyzed so far. Even for the isothermal variant of this model, existence of solutions has not yet been proved, although computational simulations are routinely launched and successfully used in applications. In particular, we extend the analysis of $[20,35]$ to the mixity-sensitive case. For other results on models for rate-independent adhesive contact, we refer to [26, 44]. The analysis of models featuring a rate-dependent evolution for the delamination variable was carried out in [4-8, 32, 33], cf. also the monographs $[10,46]$ for further references.

The initial-boundary-value problem we are going to analyze is written down in its classical formulation in Section 2. In the following lines, we just highlight the main features of the model, in particular focusing on the mixity of delamination modes. We confine ourselves to small strains and, just for the sake of notational simplicity, we restrict the analysis to the case of two (instead of several) bodies $\Omega_{+}$and $\Omega_{-}$glued together along the contact surface $\Gamma_{\mathrm{c}}$. The material in the bulk is taken to be heat conductive, and thus the system is completed by the nonlinear heat equation in a thermodynamically consistent way. The contact surface is considered infinitesimally thin, so that the thermal capacity of the adhesive is naturally neglected. The coupling of the mechanical and thermal effects thus results from thermal expansion, dissipative/adiabatic heat production/consumption (depending, in particular, on the mode mixity on $\Gamma_{\mathrm{C}}$ ), and here also from the possible dependence of the heat transfer through the contact surface $\Gamma_{\mathrm{C}}$ on the delamination itself, and on the possible slot between the bodies if the contact is debonded.

We consider an elastic response of the adhesive, and then one speaks about adhesive contact (in contrast to brittle contact where a mixity-dependent model seems to be particularly difficult to analyze). The elastic response in the adhesive will be assumed linear, determined by the (positivedefinite) matrix of elastic moduli $\mathbb{A}$. At a current time, the "volume fraction" of debonded molecular links will be "macroscopically" described by the scalar delamination parameter $z: \Gamma_{\mathrm{C}} \rightarrow[0,1]$, which can be referred to the modelling approach by M. Frémond, see [13, 14]. The state $z(x)=1$ means that the adhesive is still $100 \%$ undestroyed and thus fully effective, while the intermediate state $0<z(x)<1$ means that there are some molecular links which have been broken but the remaining ones are effective, and eventually $z(x)=0$ means that the surface is already completely debonded at $x \in \Gamma_{\mathrm{C}}$. In some simplification, based on the Griffith concept [16], it is assumed that a specific phenomenologically prescribed energy $a$ (in $\mathrm{J} / \mathrm{m}^{2}$, in 3-dimensional situations) is needed to break the macromolecular structure of the adhesive, independently of the rate of this process. Thus, delamination is a rate-independent and activated phenomenon, governed by the maximum dissipation principle, and we shall accordingly consider a rate-independent flow rule for $z$.

Let us now comment on the main new feature of the model presently analyzed, i.e., its mixitysensitivity. An immediate reflection of the standard engineering approach as, e.g., in [17, 48, 49] is to make the activation energy $a=a\left(\psi_{\mathrm{G}}\right)$ depend on the so-called mode-mixity angle $\psi_{\mathrm{G}}$. For instance, if $v_{\mathrm{C}}=(0,0,1)$ at some $x \in \Gamma_{\mathrm{C}}$ (with $v_{\mathrm{C}}$ the unit normal to $\Gamma_{\mathrm{C}}$, oriented from $\Omega_{+}$to $\Omega_{-}$), and $\mathbb{A}=\operatorname{diag}\left(\kappa_{\mathrm{n}}, \kappa_{\mathrm{t}}, \kappa_{\mathrm{t}}\right)$, the mode-mixity angle is defined as

$$
\psi_{\mathrm{G}}=\psi_{\mathrm{G}}([u]):=\arctan \sqrt{\frac{\kappa_{\mathrm{t}}\left|\llbracket u \rrbracket_{\mathrm{t}}\right|^{2}}{\kappa_{\mathrm{n}}\left|\llbracket u \rrbracket_{\mathrm{n}}\right|^{2}}}
$$

where $\llbracket u \rrbracket_{\mathrm{t}}$ and $\llbracket u \rrbracket_{\mathrm{n}}$ stand for the tangential and the normal jump of displacement. They give 
the decomposition of the jump of displacement across the boundary $\Gamma_{\mathrm{C}}$ as $\llbracket u \rrbracket=\llbracket u \rrbracket_{\mathrm{n}} v_{\mathrm{C}}+\llbracket u \rrbracket_{\mathrm{t}}$, with $\llbracket u \rrbracket_{\mathrm{n}}=\llbracket u \rrbracket \cdot v_{\mathrm{c}}$. In fact, to satisfy natural analytical requirements (viz. the continuity of the function $a_{1}$ below, cf. (1.3)), one should rather consider a suitable regularization of (1.1) to avoid discontinuity at 0 . For example, it is sufficient to take

$$
\psi_{\mathrm{G}}(\llbracket u \rrbracket)=\arctan \sqrt{\frac{\kappa_{\mathrm{t}}\left|\llbracket u \rrbracket_{\mathrm{t}}\right|^{2}}{\kappa_{\mathrm{n}}\left|\llbracket u \rrbracket_{\mathrm{n}}\right|^{2}+\epsilon}} \text { with a small } \epsilon>0 .
$$

The coefficient $\kappa_{\mathrm{t}}$ is often smaller than $\kappa_{\mathrm{n}}$, and a typical phenomenological form of $a(\cdot)$ used in engineering [17] is, e.g.,

$$
a\left(\psi_{\mathrm{G}}\right):=a_{\mathrm{I}}\left(1+\tan ^{2}\left((1-\lambda) \psi_{\mathrm{G}}\right)\right)
$$

In (1.2), $a_{\mathrm{I}}=a(0)$ is the activation threshold for delamination mode $\mathrm{I}$ and $\lambda$ is the socalled delamination-mode-sensitivity parameter. Note that moderately strong delamination-mode sensitivity occurs when the ratio $a_{\mathrm{II}} / a_{\mathrm{I}}$ is about 5-10 (where $a_{\mathrm{II}}=a\left(90^{\circ}\right)$ is the activation threshold for the pure delamination mode II). Then, one has $\lambda$ about $0.2-0.3$; cf. [49].

In the thermodynamical context, the energy $a$ needed for delamination is dissipated by the system in two ways: one part $a_{1}$ is spent to the chaotic vibration of the atomic lattice of both sides of the delaminating surface $\Gamma_{\mathrm{c}}$, which leads "macroscopically" to heat production (cf. also [44, Remark 4.2]), while another part $a_{0}$ is spent to create a new delaminated surface (or, "microscopically" speaking, to break the macromolecules of the adhesive). Thus $a=a_{0}+a_{1}$. Consistently with the dissipation, Mode II also heats up considerably more than Mode I, as experimentally documented in [34]. In view of (1.2), an option suggested already in [42] is

$$
a_{0}(\llbracket u \rrbracket):=a_{\mathrm{I}}, \quad a_{1}(\llbracket u \rrbracket):=a_{\mathrm{I}} \tan ^{2}\left((1-\lambda) \psi_{\mathrm{G}}(\llbracket u \rrbracket)\right),
$$

meaning that plain delamination does not trigger heat production at all, and only the additional dissipation related with Mode II contributes to the heat production on the delaminating surface. Moreover, taking $a_{0}$ constant reflects the phenomenon that the energy stored during delamination cannot further be changed during subsequent evolution. Anyhow, in what follows we will consider also this contribution to the stored energy dependent on $\llbracket u \rrbracket$ as it does not bring any mathematical problems and as, being premultiplied by $z$, cf. (2.12a) below, it anyhow does not influence the stored energy after complete delamination even if it depends on $\llbracket u \rrbracket$.

We summarize the features of these particular modes in Table 1.

The mathematical difficulties attached to the analysis of the PDE system for the present mixity-sensitive delamination model arise both from the proper thermodynamical coupling, and from hosting an inelastic rate-independent process on $\Gamma_{\mathrm{c}}$. Models combining thermal and rateindependent effects have already been successfully analyzed in [39] for inelastic processes in the bulk, and in [35] for surface delamination. The essential ingredient for the analysis is the satisfaction of the energy balance. In this direction, the concept of energetic solutions to rateindependent systems recently developed in [24, 27-29] and adapted to systems with inertia and viscosity in [38] appears truly essential. Here, additional difficulties are related with the mixitydependence of the dissipation, which makes it non-associative, in contrast to the mixity-insensitive case and to another model recently devised and analyzed in the isothermal case in [42, 43]. Here 
TABLE 1. Schematic summary of particular modes

\begin{tabular}{lccc}
\hline & \multicolumn{3}{c}{ Features } \\
\cline { 2 - 4 } & Energy dissipation & Heat generation & Mode-mixity angle $\psi_{\mathrm{G}}$ \\
\hline Mode I (opening) & small & small or none & $0^{\circ}$ \\
Mixed mode & moderate & moderate & in between $0^{\circ}$ and $90^{\circ}$ \\
Mode II (shearing) & large & large & $90^{\circ}$ \\
\hline
\end{tabular}

"non-associative" means that there is no unique activation threshold associated with the dissipation mechanism. (Sometimes, however, the meaning of the adjective "non-associative" rather means that the dissipative forces do not have any potential.) This analytical feature has led us to resort to a higher-order gradient in the momentum equation via the concept of the so-called hyperstresses, already justified and used in the theoretical-mechanical literature, in relation with the concept of so-called nonsimple materials, cf. e.g. [15, 31, 50]. Such a regularization brings various inevitable technicalities into the classical formulation of the problem, cf. (2.6) and (3.3) later on.

The main result of this paper is the existence of solutions to the initial-boundary value problem associated with the mixity-sensitive model under investigation. The proof is performed by passing to the limit in a suitably devised semi-implicit discretization scheme, cf. (5.4). Let us mention that such a kind of scheme (already applied in [21, Sect.3.1] for a special dynamic isothermal fracture problem) leads to considerable analytical simplifications, in comparison with the fully implicit scheme used in [35]. In the existence proof we shall distinguish the dynamic case, involving inertial terms in the momentum equation, and the quasistatic one, where inertia is neglected. In the latter situation, we will be able to tackle fairly general contact conditions for the displacement variable $u$, in particular including (a generalization of) the Signorini frictionless contact law. For further explanations and comments, we refer to Remark 4.3.

Let us emphasize, not to create any confusion, that analytically the problem exhibits a lot of difficulties and there is probably not much freedom in developing a model that would be mathematically justified, although engineers routinely run computational simulations where e.g. the mode-mixity angle $\psi_{\mathrm{G}}$ depends on traction stresses, which hardly can guarantee continuity of the corresponding Nemytskiir mapping in any sense. Having doubly nonlinear structure of delamination with constraints both on $\dot{z}$ and on $z$, introducing a (small) viscosity into the flow rule does not seem trivial or even possible, cf. [19]. Yet, considering the flow rule for $z$ without viscosity (i.e., rate-independent), $\dot{z}$ is controlled only as a measure on $\bar{\Sigma}_{\text {c }}$. Then, in our modesensitive delamination, $u$ must have traces on $\Sigma_{\mathrm{C}}$ continuous on $\bar{\Sigma}_{\mathrm{C}}$, cf. (6.6) below. It is in order to have this, in our 3-dimensional framework, that we need to resort to the concept of nonsimple materials (i.e., hyperstresses). More precisely, simple materials would work but only in (practically not interesting) 1-dimensional problems. Having elastic hyperstresses, we also need corresponding viscous hyperstresses, which makes the acceleration in duality with velocity and thus guarantees the energy preservation. This is how the rigorously justified mode-sensitive model presented in Section 2 has been gradually built, possibly without much freedom for other options. And, after all, still a practically important case (namely inertia combined with unilateral contact) remains uncovered, which documents nontriviality of the whole issue. 
The plan of the paper is as follows: in Section 2 we formulate the initial-boundary-value problem in its classical formulation. We also briefly sketch its derivation, referring to [35] for more details. Hence, in Sec. 3, after introducing a suitable transformation for the heat equation, we advance a suitably devised weak formulation of our PDE system, and comment on its relation to the classical formulation of Sec. 2. We state our main existence result in Sec. 4, and set up the approximation via a semi-implicit time-discretization scheme in Sec. 5. For the discrete solutions, suitable a priori estimates are obtained, which allow us to pass to the limit in the time-discrete approximation, and conclude the existence of solutions in Sec. 6.

\section{The model and its derivation}

Hereafter, we suppose that the elastic body occupies a bounded Lipschitz domain $\Omega \subset \mathbb{R}^{3}$ and is composed by two disjoint Lipschitz subdomains $\Omega_{+}$and $\Omega_{-}$, by $\Gamma_{\mathrm{C}}$ their common boundary, which represents a prescribed surface where delamination may occur, i.e.

$$
\Omega=\Omega_{+} \cup \Gamma_{\mathrm{C}} \cup \Omega_{-} .
$$

We denote by $v$ the outward unit normal to $\partial \Omega$, and by $v_{\mathrm{C}}$ the unit normal to $\Gamma_{\mathrm{C}}$, which we consider oriented from $\Omega_{+}$to $\Omega_{-}$. Moreover, given $v \in W^{1,2}\left(\Omega \backslash \Gamma_{\mathrm{C}}\right), v^{+}$(respectively, $v^{-}$) signifies the restriction of $v$ to $\Omega_{+}$(to $\Omega_{-}$, resp.). We further suppose that the boundary $\partial \Omega$ of $\Omega$ splits as

$$
\partial \Omega=\Gamma_{\mathrm{D}} \cup \Gamma_{\mathrm{N}} \cup \Gamma_{0}
$$

with $\Gamma_{\mathrm{D}}$ and $\Gamma_{\mathrm{N}}$ open subsets in the relative topology of $\partial \Omega$, disjoint one from each other, and $\Gamma_{0}$ with zero 2-dimensional measure. Considering $T>0$ a fixed time horizon, we set

$$
\begin{gathered}
Q:=(0, T) \times \Omega, \quad \Sigma:=(0, T) \times \partial \Omega, \quad \Sigma_{\mathrm{C}}:=(0, T) \times \Gamma_{\mathrm{C}}, \quad \Sigma_{\mathrm{D}}:=(0, T) \times \Gamma_{\mathrm{D}}, \\
\Sigma_{\mathrm{N}}:=(0, T) \times \Gamma_{\mathrm{N}} .
\end{gathered}
$$

For the reader's convenience, let us summarize the basic notation used in Table 2.

The state is formed by the triple $(u, \theta, z)$. We use Kelvin-Voigt's rheology and thermal expansion. As a further contribution to the stress $\sigma:(0, T) \times \Omega \rightarrow \mathbb{R}^{3 \times 3}$, we also consider the hyperstress $\mathfrak{h}:(0, T) \times \Omega \rightarrow \mathbb{R}^{3 \times 3 \times 3}$, which accounts for "capillarity-like" effects supposed to occur in so-called nonsimple materials. Mimicking Kelvin-Voigt's rheology, we incorporate the corresponding dissipation contribution to the hyperstress $\mathfrak{h}$. Hence we assume the stress $\sigma$ : $(0, T) \times \Omega \rightarrow \mathbb{R}^{3 \times 3}$ in the form:

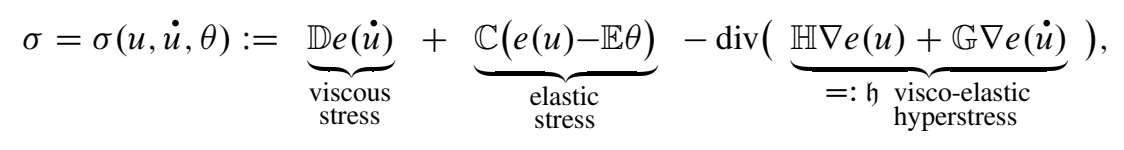

The $\mathbb{G}$-term will ensure that the acceleration $\ddot{u}$ is in duality with the velocity $\dot{u}$, which is needed if $\varrho>0$ is considered. Furthermore, we shall denote by $T=T(u, v, \theta, n)$ the traction stress on some two-dimensional surface $\Gamma$ with outward unit normal $n$, i.e.

$$
T(u, \dot{u}, \theta, n):=\left.\sigma(u, \dot{u}, \theta)\right|_{\Gamma} n .
$$

Later, we shall take either $\Gamma=\Gamma_{\mathrm{N}}$ and $n=v$, or $\Gamma=\Gamma_{\mathrm{C}}$ and $n=v_{\mathrm{c}}$. 
TABLE 2. Summary of the main notation used through the paper

\begin{tabular}{ll}
\hline$u: \Omega \backslash \Gamma_{\mathrm{C}} \rightarrow \mathbb{R}^{3}$ displacement, & $\mathbb{B}:=\mathbb{C} \mathbb{E}$, \\
$\theta: \Omega \backslash \Gamma_{\mathrm{C}} \rightarrow(0,+\infty)$ absolute temperature, & $\mathbb{A} \in \mathbb{R}^{3 \times 3}$ elastic coefficients of the adhesive, \\
$z: \Gamma_{\mathrm{C}} \rightarrow[0,1]$ delamination variable, & $\varrho>0$ mass density, \\
$e=e(u)=\frac{1}{2} \nabla u^{\top}+\frac{1}{2} \nabla u$ small strain tensor, & $c_{\mathrm{V}}=c_{\mathrm{V}}(\theta)$ heat capacity, \\
$\llbracket u \rrbracket=\left.u^{+}\right|_{\mathrm{C}}-\left.u^{-}\right|_{\Gamma_{\mathrm{C}}}$ jump of $u$ across $\Gamma_{\mathrm{C}}$, & $a_{0}=a_{0}(\llbracket u \rrbracket)$ energy stored on $\Gamma_{\mathrm{C}}$, \\
$\sigma$ stress tensor, & $a_{1}=a_{1}(\llbracket u \rrbracket)$ energy dissipated on $\Gamma_{\mathrm{C}}$, \\
$\mathfrak{h}$ visco-elastic hyperstress, & $\eta=\eta(\llbracket u \rrbracket, z)$ heat-transfer coefficient on $\Gamma_{\mathrm{C}}$, \\
$\vartheta$ rescaled temperature (enthalpy), & $F: Q \rightarrow \mathbb{R}^{3}$ applied bulk force, \\
$\mathbb{C} \in \mathbb{R}^{3^{4}}$ elasticity constants, & $w_{\mathrm{D}}$ prescribed boundary displacement, \\
$\mathbb{D} \in \mathbb{R}^{3^{4}}$ viscosity constants, & $f: \Sigma_{\mathrm{N}} \rightarrow \mathbb{R}^{3}$ applied traction, \\
$\mathbb{H} \in \mathbb{R}^{3^{6}}$ elasticity constants for hyperstress, & $G: Q \rightarrow \mathbb{R}$ bulk heat source, \\
$\mathbb{G} \in \mathbb{R}^{3^{6}}$ viscosity constants for hyperstress, & $g: \Sigma \rightarrow \mathbb{R}$ external heat flux, \\
$\mathbb{K}=\mathbb{K}^{(}(e, \theta) \in \mathbb{R}^{3 \times 3}$ heat-conduction coefficients, & $\psi$ 's (bulk and surface) free energies, \\
$\mathbb{E} \in \mathbb{R}^{3 \times 3}$ thermal-expansion coefficients, & $\zeta$ 's (pseudo)potentials of dissipative forces, \\
& $\xi$ 's (bulk and surface) rates of dissipation. \\
\end{tabular}

We address a generalization of the standard frictionless Signorini conditions on $\Gamma_{\mathrm{C}}$ for the displacement $u$. This is rendered through a closed, convex cone $K(x) \subset \mathbb{R}^{3}$, possibly depending on $x \in \Gamma_{\mathrm{C}}$. In terms of the general cone-valued mapping $K: \Gamma_{\mathrm{C}} \rightrightarrows \mathbb{R}^{3}$, in the case of no adhesion, the boundary conditions on $\Gamma_{\mathrm{C}}$ can be given in the complementarity form as

$$
\left.\begin{array}{rll}
\llbracket u \rrbracket & \succeq & 0, \\
T\left(u, \dot{u}, \theta, v_{\mathrm{C}}\right) & \succeq & 0, \\
T\left(u, \dot{u}, \theta, v_{\mathrm{C}}\right) \cdot \llbracket u \rrbracket & = & 0
\end{array}\right\} \quad \text { on } \Gamma_{\mathrm{C}} .
$$

In (2.3), $\succeq$ is the ordering induced by the mapping $K: \Gamma_{\mathrm{C}} \rightrightarrows \mathbb{R}^{3}$, in the sense that, for $v_{1}, v_{2}$ : $\Gamma_{\mathrm{C}} \rightarrow \mathbb{R}^{3}$,

$$
v_{1} \succeq v_{2} \text { if and only if } v_{1}(x)-v_{2}(x) \in K(x) \text { for a.a. } x \in \Gamma_{\mathrm{C}} .
$$

Likewise, $\stackrel{*}{\Xi}$ is the dual ordering induced by the negative polar cone to $K$, viz. for $\zeta_{1}, \zeta_{2}: \Gamma_{\mathrm{C}} \rightarrow \mathbb{R}^{3}$,

$$
\zeta_{1} \stackrel{*}{\succeq} \zeta_{2} \text { if and only if } \zeta_{1}(x) \cdot v \geqslant \zeta_{2}(x) \cdot v \text { for all } v \in K(x) \text {, for a.a. } x \in \Gamma_{\mathrm{C}} .
$$

Possible choices for the cone-valued mapping $K: \Gamma_{\mathrm{C}} \rightrightarrows \mathbb{R}^{3}$ are

$$
\begin{aligned}
& K(x)=\left\{v \in \mathbb{R}^{3} ; v \cdot v_{\mathrm{C}}(x) \geqslant 0\right\} \text { for a.a. } x \in \Gamma_{\mathrm{C}}, \quad \text { or } \\
& K(x)=\mathbb{R}^{3} \text { for a.a. } x \in \Gamma_{\mathrm{C}} .
\end{aligned}
$$

In the case (2.5a), conditions (2.3) reduce to the standard unilateral frictionless Signorini contact conditions for the normal displacement. In the case (2.5b), the complementarity problem (2.3) translates into $T\left(u, \dot{u}, \theta, v_{\mathrm{c}}\right)=0$ on $\Gamma_{\mathrm{C}}$. Thus, (2.5b) does not allow for any interaction of the bodies $\Omega_{+}$and $\Omega_{-}$after a complete delamination. In fact, this model is very simplified because it does not prevent possible interpenetration and delamination can be thus triggered, rather unphysically, by mere compression. Nevertheless, a model like this may be feasible in specific situations 
and neglecting the interpenetration after developed cracks is often accepted in the mathematical literature, cf., e.g., [9]. Note that, in (2.5b), $K(x)$ is a linear manifold for a.a. $x \in \Gamma_{\mathrm{c}}$. Indeed, later on (cf. Thm. 4.2) we shall have to assume the latter property in the case $\varrho>0$. Let us finally mention that, the complementarity problem (2.3) will take a more complicated form if adhesion is involved, cf. (2.6i-k) below.

Classical formulation of the adhesive contact problem. Beside the force equilibrium coupled with the heat equation inside $Q \backslash \Sigma_{\mathrm{c}}$ and supplemented with standard boundary conditions, we have two complementarity problems on $\Sigma_{\mathrm{C}}$. Altogether, we have the boundary-value problem

$$
\begin{array}{ll}
\varrho \ddot{u}-\operatorname{div}(\mathbb{D} e(\dot{u})+\mathbb{C} e(u)-\mathbb{B} \theta-\operatorname{div} \mathfrak{h})=F, \quad \mathfrak{h}=\mathbb{H} \nabla e(u)+\mathbb{G} \nabla e(\dot{u}) & \text { in } Q \backslash \Sigma_{\mathrm{C}}, \\
c_{\mathrm{v}}(\theta) \dot{\theta}-\operatorname{div}(\mathbb{K}(e(u), \theta) \nabla \theta)=\mathbb{D} e(\dot{u}): e(\dot{u})-\theta \mathbb{B}: e(\dot{u})+\mathbb{G} \nabla e(\dot{u}): \nabla e(\dot{u}) & \text { in } Q \backslash \Sigma_{\mathrm{C}}, \\
u=0 & \text { on } \Sigma_{\mathrm{D}}, \\
T(u, \dot{u}, \theta, v)-\operatorname{div}_{\mathrm{S}}(\mathfrak{h} \cdot v)=f & \text { on } \Sigma_{\mathrm{N}}, \\
(\mathbb{K}(e(u), \theta) \nabla \theta) v=g & \text { on } \Sigma, \\
\mathfrak{h}:(v \otimes v)=0 & \text { on } \Sigma_{\mathrm{D}} \cup \Sigma_{\mathrm{N}}, \\
\llbracket \mathbb{D} e(\dot{u})+\mathbb{C} e(u)-\mathbb{B} \theta-\operatorname{div} \mathfrak{h} \rrbracket v_{\mathrm{C}}-\operatorname{div}_{\mathrm{S}}\left(\llbracket \mathfrak{h} \rrbracket v_{\mathrm{C}}\right)=0 & \text { on } \Sigma_{\mathrm{C}}, \\
\mathfrak{h}^{+}:\left(v_{\mathrm{C}} \otimes v_{\mathrm{C}}\right)=\mathfrak{h}^{-}:\left(v_{\mathrm{C}} \otimes v_{\mathrm{C}}\right)=0 & \text { on } \Sigma_{\mathrm{C}}, \\
\llbracket u \rrbracket \succeq 0 & \text { on } \Sigma_{\mathrm{C}}, \\
T\left(u, \dot{u}, \theta, v_{\mathrm{C}}\right)-\operatorname{div}_{\mathrm{S}}\left(\mathfrak{h} \cdot v_{\mathrm{C}}\right)+z \mathbb{A} \llbracket u \rrbracket-z a_{0}^{\prime}(\llbracket u \rrbracket) \succeq 0 & \text { on } \Sigma_{\mathrm{C}}, \\
\left(T\left(u, \dot{u}, \theta, v_{\mathrm{C}}\right)-\operatorname{div}_{\mathrm{S}}\left(\mathfrak{h} \cdot v_{\mathrm{C}}\right)+z \mathbb{A} \llbracket u \rrbracket-z a_{0}^{\prime}(\llbracket u \rrbracket)\right) \cdot \llbracket u \rrbracket=0 & \text { on } \Sigma_{\mathrm{C}}, \\
\dot{z} \leqslant 0 & \text { on } \Sigma_{\mathrm{C}}, \\
d \leqslant a_{0}(\llbracket u \rrbracket)+a_{1}(\llbracket u \rrbracket) & \text { on } \Sigma_{\mathrm{C}}, \\
\dot{z}\left(d-a_{0}(\llbracket u \rrbracket)-a_{1}(\llbracket u \rrbracket)\right)=0 & \text { on } \Sigma_{\mathrm{C}}, \\
d \in \partial I_{[0,1]}(z)+\frac{1}{2} \mathbb{A} \llbracket u \rrbracket \cdot \llbracket u \rrbracket & \text { on } \Sigma_{\mathrm{C}}, \\
\frac{1}{2}\left(\left.\mathbb{K}(e(u), \theta) \nabla \theta\right|_{\Gamma_{\mathrm{C}}} ^{+}+\left.\mathbb{K}(e(u), \theta) \nabla \theta\right|_{\Gamma_{\mathrm{C}}} ^{-}\right) \cdot v_{\mathrm{C}}+\eta(\llbracket u \rrbracket, z) \llbracket \theta \rrbracket=0 & \text { on } \Sigma_{\mathrm{C}}, \\
\llbracket \mathbb{K}(e(u), \theta) \nabla \theta \rrbracket \cdot v_{\mathrm{C}}=-a_{1}(\llbracket u \rrbracket) \dot{z} & \text { on } \Sigma_{\mathrm{C}},
\end{array}
$$

where $\vdots$ denotes the tensorial product involving summation over 3 indices, and we have used the notation $\mathfrak{h}^{+}=\mathfrak{h} \mid \Omega_{+}$and $\mathfrak{h}^{-}=\mathfrak{h} \mid \Omega_{-}$. In (2.6d), we denoted by div $\operatorname{s}_{\mathrm{S}}$ the two-dimensional "surface divergence", defined by $\operatorname{div}_{\mathrm{S}}:=\operatorname{tr}\left(\nabla_{\mathrm{S}}\right.$ ), where $\operatorname{tr}$ is the trace operator (of a $2 \times 2$ matrix), and $\nabla_{\mathrm{S}}$ denotes the tangential derivative, defined by $\nabla_{\mathrm{s}} v=\nabla v-\frac{\partial v}{\partial v} v$. As to the involved tensorial symbols,

$\mathbb{K}=\mathbb{K}(e, \theta) \quad$ is a 2 nd-order positive definite tensor,

i.e., a $3 \times 3$-matrix, while

$$
\begin{aligned}
& \mathbb{C}, \mathbb{D}: \mathbb{R}_{\text {sym }}^{3 \times 3} \rightarrow \mathbb{R}_{\mathrm{sym}}^{3 \times 3} \quad \text { are 4th-order positive definite tensors, and } \\
& \forall i, j, k, l=1, \ldots, 3: \quad \mathbb{C}_{i j k l}=\mathbb{C}_{j i k l}=\mathbb{C}_{k l i j} \text { and } \mathbb{D}_{i j k l}=\mathbb{D}_{j i k l}
\end{aligned}
$$


in the sense that the form $e \mapsto \mathbb{C} e: e$ is convex, and similarly for $\mathbb{D}$. In particular, these symmetries ensure that both $\mathbb{C} e(u)$ and $\mathbb{D} e(u)$ are $\mathbb{R}_{\text {sym }}^{3 \times 3}$-valued, and the operator $\operatorname{div} \mathbb{C} e(u)$ has a potential. Analogously, for the higher-order terms we suppose that

$$
\begin{aligned}
& \mathbb{H}, \mathbb{G}: \mathbb{R}^{3 \times 3 \times 3} \rightarrow \mathbb{R}^{3 \times 3 \times 3} \text { are 6th-order positive definite tensors, and } \\
& \forall i, j, k, l, m, n=1, \ldots, 3: \quad \mathbb{H}_{i j k l m n}=\mathbb{H}_{i k j l m n}=\mathbb{H}_{l m n i j k} \text { and } \mathbb{G}_{i j k l m n}=\mathbb{G}_{i k j l m n} .
\end{aligned}
$$

In particular, this ensures that both $\operatorname{div} \mathbb{H} \nabla e(u)$ and $\operatorname{div} \mathbb{G} \nabla e(u)$ are $\mathbb{R}_{\text {sym }}^{3 \times 3}$-valued, and the operator $\operatorname{div}^{2} \mathbb{H} \nabla e(u)$ has a potential.

The complementarity problem (2.6i)-(2.6k) describes general, possibly unilateral (depending on the choice of the mapping $K: \Gamma_{\mathrm{c}} \rightrightarrows \mathbb{R}^{3}$ ) contact. Indeed, for $\mathfrak{h}=0$ and $z=0$ (i.e., no hyperstress contribution, and complete delamination), (2.6i)-(2.6k) reduce to relations (2.3), which in turn generalize the Signorini conditions. For later reference, we point out that the complementarity conditions (2.6i)-(2.6k) are equivalent to the subdifferential inclusion

$$
\partial I_{K}(\llbracket u \rrbracket)+T\left(u, \dot{u}, \theta, v_{\mathrm{c}}\right)+z \mathbb{A} \llbracket u \rrbracket-z a_{0}^{\prime}(\llbracket u \rrbracket) \ni 0 \text { on } \Sigma_{\mathrm{c}},
$$

which features the indicator functional $I_{K}: L^{2}\left(\Gamma_{\mathrm{c}} ; \mathbb{R}^{3}\right) \rightarrow[0,+\infty]$ associated with the multivalued mapping $K: \Gamma_{\mathrm{c}} \rightrightarrows \mathbb{R}^{3}$, viz.

$$
I_{K}(v)=\int_{\Gamma_{\mathrm{C}}} I_{K(x)}(v(x)) \mathrm{d} S \quad \text { for all } v \in L^{2}\left(\Gamma_{\mathrm{C}} ; \mathbb{R}^{3}\right),
$$

and its subdifferential (in the sense of convex analysis) $\partial I_{K}: L^{2}\left(\Gamma_{\mathrm{c}} ; \mathbb{R}^{3}\right) \rightrightarrows L^{2}\left(\Gamma_{\mathrm{c}} ; \mathbb{R}^{3}\right)$.

In turn, adhesive contact results from the complementarity conditions (2.61)-(2.60), which can be reformulated as the flow rule

$$
\partial I_{(-\infty, 0]}(\dot{z})+\partial I_{[0,1]}(z)+\frac{1}{2} \mathbb{A} \llbracket u \rrbracket \cdot \llbracket u \rrbracket-a_{0}(\llbracket u \rrbracket)-a_{1}(\llbracket u \rrbracket) \ni 0 \text { in } \Sigma_{\mathrm{C}},
$$

with the indicator functions $I_{(-\infty, 0]}, I_{[0,1]}: \mathbb{R} \rightarrow[0,+\infty]$ and their (convex analysis) subdifferentials $\partial I_{(-\infty, 0]}, \partial I_{[0,1]}: \mathbb{R} \rightrightarrows \mathbb{R}$.

Some comments on the derivation of the model. In [35], a thorough derivation of the analogue of system (2.6), in the case when the mode-mixity dependence is neglected in the model, has been developed. Therefore, we refer the reader to [35, Sec. 3] for all details, and in the next lines we just highlight the main differences between the system considered in [35] and the present (2.6). Namely, here the free energy is enhanced by the higher-order term $\frac{1}{2} \mathbb{H} \nabla e(u): \nabla e(u)$, the dissipation energy in the bulk is enhanced by $\mathbb{G} \nabla e(\dot{u}): \nabla e(\dot{u})$, and on the delamination surface $\Gamma_{\mathrm{C}}$ we have $a_{0}$ and $a_{1}$ depending on $\llbracket u \rrbracket$.

More specifically, we consider the free energy, the dissipation rate, and the (pseudo)potential of dissipative forces in the bulk given by

$$
\begin{aligned}
& \psi^{\text {bulk }}(e, \nabla e, \theta)=\frac{1}{2} \mathbb{C}(e-\mathbb{E} \theta):(e-\mathbb{E} \theta)+\frac{1}{2} \mathbb{H} \nabla e: \nabla e-\frac{\theta^{2}}{2} \mathbb{B}: \mathbb{E}-\psi_{0}(\theta), \\
& \xi^{\text {bulk }}(\dot{e}, \nabla \dot{e})=2 \zeta_{2}(\dot{e}, \nabla \dot{e}) \quad \text { with } \zeta_{2}(\dot{e}, \nabla \dot{e}):=\frac{1}{2} \mathbb{D} \dot{e}: \dot{e}+\frac{1}{2} \mathbb{G} \nabla \dot{e}: \nabla \dot{e},
\end{aligned}
$$


where $\psi_{0}:(0,+\infty) \rightarrow \mathbb{R}$ is a strictly convex function. The free energy and the dissipation rate on the contact surface are

$$
\begin{aligned}
& \psi^{\text {surf }}(v, z)= \begin{cases}z\left(\frac{1}{2} \mathbb{A} v \cdot v-a_{0}(v)\right) & \text { if } v \succeq 0 \text { and } 0 \leqslant z \leqslant 1, \\
+\infty & \text { otherwise, }\end{cases} \\
& \xi^{\text {surf }}(v, \dot{z})=\zeta_{1}(v, \dot{z}):= \begin{cases}a_{1}(v)|\dot{z}| & \text { if } \dot{z} \leqslant 0, \\
+\infty & \text { otherwise. }\end{cases}
\end{aligned}
$$

The overall free energy and the (pseudo)potential of dissipative forces are then

$$
\begin{aligned}
& \Psi(u, z, \theta)=\int_{\Omega \backslash \Gamma_{\mathrm{C}}} \psi^{\text {bulk }}(e(u), \nabla e(u), \theta) \mathrm{d} x+\int_{\Gamma_{\mathrm{C}}} \psi^{\text {surf }}(\llbracket u \rrbracket, z) \mathrm{d} S, \\
& \Xi(u ; \dot{u}, \dot{z})=\int_{\Omega \backslash \Gamma_{\mathrm{C}}} \xi^{\text {bulk }}(e(\dot{u}), \nabla e(\dot{u})) \mathrm{d} x+\int_{\Gamma_{\mathrm{C}}} \xi^{\text {surf }}(\llbracket u \rrbracket, \dot{z}) \mathrm{d} S,
\end{aligned}
$$

respectively. Considering the specific kinetic energy $\frac{1}{2} \varrho|v|^{2}$ (with $\varrho>0$ the mass density), for all $v \in L^{2}(\Omega)$ we define the overall kinetic energy $T_{\text {kin }}$ and the external mechanical load $L$ by

$$
T_{\text {kin }}(v):=\frac{1}{2} \int_{\Omega} \varrho|v|^{2} \mathrm{~d} x \quad \text { and } \quad\langle L(t), u\rangle:=\int_{\Omega} F(t) \cdot u \mathrm{~d} x+\int_{\Gamma_{\mathrm{N}}} f \cdot u \mathrm{~d} S .
$$

The mechanical part of system (2.6), i.e. equations (2.6a, c, d, f-o), is then just the classical formulation of the abstract evolutionary system

$$
\partial T_{\text {kin }}(\ddot{u}(t))+\partial_{(\dot{u}, \dot{z})} \Xi(u(t) ; e(\dot{u}(t)), \dot{z}(t))+\partial_{(u, z)} \Psi(u(t), z(t), \theta(t)) \ni L(t) \text { for } t \in(0, T),
$$

where $\partial$ denotes the (convex analysis) subdifferential of the functionals $\Xi$ and $\Psi$, w.r.t. suitable topologies which we do not specify. The remaining equations in (2.6) yield the heat-transfer problem, i.e. (2.6b, e, p, q). Its derivation proceeds standardly from postulating the entropy $s$ by the so-called Gibbs' relation $s=-\Psi_{\theta}^{\prime}(u, z, \theta)$, viz. $\langle s, \tilde{\theta}\rangle=-\Psi_{\theta}^{\prime}(u, z, \theta ; \tilde{\theta})$ for all $\tilde{\theta}$, where $\Psi_{\theta}^{\prime}(u, z, \theta ; \tilde{\theta})$ is the directional derivative of $\Psi$ at $(u, z, \theta)$ in the direction $\tilde{\theta}$. This yields the entropy in the bulk as

$$
s=s(\theta, e)=-\frac{\partial \psi^{\mathrm{bulk}}}{\partial \theta}(e(u), \theta)=\mathbb{B}: e(u)+\psi_{0}^{\prime}(\theta) .
$$

Further, we use the so-called entropy equation

$$
\theta \dot{s}=\xi^{\text {bulk }}(e(\dot{u}))-\operatorname{div} j
$$

Substituting $\dot{s}=\mathbb{B}: e(\dot{u})-\psi_{0}^{\prime \prime}(\theta) \dot{\theta}$, cf. (2.17), into the entropy equation (2.18) yields the heat equation

$$
c_{\mathrm{v}}(\theta) \dot{\theta}+\operatorname{div} j=2 \zeta_{2}(e(\dot{u}))-\theta \mathbb{B}: e(\dot{u}) \quad \text { with } \quad c_{\mathrm{v}}(\theta)=\theta \psi_{0}^{\prime \prime}(\theta) .
$$

Hence, assuming the constitutive relation $j:=-\mathbb{K}(e(u), \theta) \nabla \theta$ for the heat flux, i.e. Fourier's law in an anisotropic medium, one obtains the heat equation in the form (2.6b). 
Similar, but simpler thermodynamics can also be seen on the contact boundary by involving $\psi^{\text {surf }}$ and $\xi^{\text {surf }}$. Since $(2.12 \mathrm{a})$ is independent of temperature, the "boundary entropy" $=-\frac{\partial}{\partial \theta} \psi^{\text {surf }}$ is simply zero, and the corresponding entropy equation reduces to $0=\xi^{\text {surf }}(\dot{z})-\llbracket j \rrbracket$ (as an analog of (2.18)), which then results in (2.6q). Incorporating the analog of the phenomenological Fourier's law, we arrive at $(2.6 \mathrm{p})$.

We emphasize that the model is thermodynamically consistent, in the sense that it conserves the total energy, i.e., here

$$
\begin{gathered}
\frac{\mathrm{d}}{\mathrm{d} t}(\underbrace{\int_{\Omega \backslash \Gamma_{\mathrm{C}}} \frac{\varrho}{2}|\dot{u}|^{2}+\frac{1}{2} \mathbb{C} e(u): e(u)+\frac{1}{2} \mathbb{H} \nabla e(u) \vdots \nabla e(u)+h(\theta) \mathrm{d} x}_{\text {kinetic, elastic, and thermal energies }} \\
+\underbrace{\int_{\Gamma_{\mathrm{C}}} \frac{z}{2} \mathbb{A} \llbracket u \rrbracket \cdot \llbracket u \rrbracket-a_{0}(\llbracket u \rrbracket) z \mathrm{~d} S}_{\begin{array}{c}
\text { mechanical energy } \\
\text { in the adhesive }
\end{array}}) \\
=\underbrace{\int_{\Omega} F \cdot \dot{u} \mathrm{~d} x}_{\begin{array}{c}
\text { power of bulk } \\
\text { mechanical load }
\end{array}}+\underbrace{\int_{\Gamma} g \mathrm{~d} S+\int_{\Gamma_{\mathrm{N}}} f \cdot \dot{u} \mathrm{~d} S}_{\begin{array}{c}
\text { power of surface heat } \\
\text { and mechanical load }
\end{array}},
\end{gathered}
$$

and it satisfies the Clausius-Duhem's entropy inequality:

$$
\frac{\mathrm{d}}{\mathrm{d} t} \int_{\Omega} s \mathrm{~d} x=\int_{\Omega} \frac{\operatorname{div}(\mathbb{K} \nabla \theta)}{\theta} \mathrm{d} x=\int_{\Omega} \frac{\mathbb{K} \nabla \theta \cdot \nabla \theta}{\theta^{2}} \mathrm{~d} x+\int_{\partial \Omega} \frac{g}{\theta} \mathrm{d} S \geqslant 0,
$$

as well as non-negativity of the temperature under suitable natural conditions, cf. Theorem 4.2.

REMARK 2.1 The general theory of heat transfer in the bulk and across interfaces rather uses the coldness $1 / \theta$ as a driving force than temperature $\theta$ itself, as discussed in [18], cf. also [25]. Since we allow for a dependence of $\mathbb{K}$ on $\theta$, we can easily write $\mathbb{K}(e, \theta) \nabla \theta$ as $-\tilde{\mathbb{K}}(e, \theta) \nabla(1 / \theta)$ for $\tilde{\mathbb{K}}(e, \theta):=\mathbb{K}(e, \theta) \theta^{2}$. The transmission condition (2.6p) should then contain the term $-\tilde{\eta}(\llbracket u \rrbracket, z) \llbracket 1 / \theta \rrbracket$ rather than $\eta(\llbracket u \rrbracket, z) \llbracket \theta \rrbracket$. Obviously, our term $\eta(\llbracket u \rrbracket, z) \llbracket \theta \rrbracket$ could be written as $-\tilde{\eta}\left(\llbracket u \rrbracket, z,\left.\theta^{+}\right|_{\Gamma_{\mathrm{C}}},\left.\theta^{-}\right|_{\Gamma_{\mathrm{C}}}\right) \llbracket 1 / \theta \rrbracket$ with $\tilde{\eta}\left(\llbracket u \rrbracket, z, \theta^{+}, \theta^{-}\right):=\eta(\llbracket u \rrbracket, z) \theta^{+} \theta^{-}$. Conversely, $\tilde{\eta}(\llbracket u \rrbracket, z)$ would yield $\eta\left(\llbracket u \rrbracket, z, \theta^{+}, \theta^{-}\right)=\tilde{\eta}(\llbracket u \rrbracket, z) /\left(\theta^{+} \theta^{-}\right)$. This reveals that we should allow for the coefficient $\eta$ to depend on the traces of $\left.\theta^{+}\right|_{\Gamma_{\mathrm{C}}}$ and $\left.\theta^{-}\right|_{\Gamma_{\mathrm{C}}}$ as well. This additional dependence, also subject to the transformation in Sect. 3 below, would lead to rather straightforward modifications in a lot of formulas, assumptions, estimates, and limit passages. The latter should anyhow be possible due to the strong convergence of the traces of the (transformed) temperature, cf. (6.9) below. That is why, we dare omit to explore this generalization.

REMARK 2.2 Alternatively, in the lines of [6] and [25, Sec. 4.2], we could write entropy production due to heat transfer as a part of the dissipation potential, so that the heat flux can be seen as a gradient of this augmented potential with respect to the temperature gradients (or differences). Namely, we could incorporate the contribution $\frac{1}{2} \mathbb{K}(e, \theta) \nabla \theta \cdot \nabla \theta$ into $\xi^{\text {bulk }}$ in $(2.11 \mathrm{~b})$, and $\frac{1}{2} \eta(\llbracket u \rrbracket, z)|\llbracket \theta \rrbracket|^{2}$ into $\xi^{\text {surf }}$ in $(2.12 \mathrm{~b})$. Then the heat flux $\mathbb{K}(e, \theta) \nabla \theta$ would turn out to be $\partial \xi^{\text {bulk }} / \partial(\nabla \theta)$ and the boundary heat flux $\eta(\llbracket u \rrbracket, z) \llbracket \theta \rrbracket$ would be $\partial \xi^{\text {surf }} / \partial(\llbracket \theta \rrbracket)$. 


\section{Enthalpy transformation and energetic solution}

In what follows we are going to tackle a reformulation of the PDE system (2.6), in which we replace the heat equation (2.6b) with an enthalpy equation. Namely, we switch from the absolute temperature $\theta$, to the enthalpy $\vartheta$, defined via the so-called enthalpy transformation, viz.

$$
\vartheta=h(\theta):=\int_{0}^{\theta} c_{\mathrm{v}}(r) \mathrm{d} r .
$$

Thus, $h$ is a primitive function of $c_{\mathrm{v}}$, normalized in such a way that $h(0)=0$. We will assume in (4.1a) below that $c_{\mathrm{v}}$ is strictly positive, hence $h$ is strictly increasing. Thus, we are entitled to define

$$
\Theta(\vartheta):=\left\{\begin{array}{ll}
h^{-1}(\vartheta) & \text { if } \vartheta \geqslant 0, \\
0 & \text { if } \vartheta<0,
\end{array} \quad \mathcal{K}(e, \vartheta):=\frac{\mathbb{K}(e, \Theta(\vartheta))}{c_{\mathrm{v}}(\Theta(\vartheta))},\right.
$$

where $h^{-1}$ denotes the inverse function to $h$.

With transformation (3.1) and the related (3.2), also taking into account the subdifferential formulations (2.8) and (2.10), the PDE system (2.6) turns into

$$
\begin{aligned}
& \varrho \ddot{u}-\operatorname{div}(\mathbb{D} e(\dot{u})+\mathbb{C} e(u)-\mathbb{B} \Theta(\vartheta)-\operatorname{div} \mathfrak{h})=F, \mathfrak{h}=\mathbb{H} \nabla e(u)+\mathbb{G} \nabla e(\dot{u}) \\
& \dot{\vartheta}-\operatorname{div}(\mathcal{K}(e(u), \vartheta) \nabla \vartheta)=\mathbb{D} e(\dot{u}): e(\dot{u})-\Theta(\vartheta) \mathbb{B}: e(\dot{u})+\mathbb{G} \nabla e(\dot{u}): \nabla e(\dot{u}) \quad \text { in } Q \backslash \Sigma_{\mathrm{C}} \text {, } \\
& u=0 \\
& \text { on } \Sigma_{\mathrm{D}} \text {, } \\
& (\mathcal{K}(e(u), \vartheta) \nabla \vartheta) v=g \\
& \tau(u, \dot{u}, \vartheta, v)-\operatorname{div}_{\mathrm{S}}(\mathfrak{h} \cdot v)=f \\
& \} \text { on } \Sigma_{\mathrm{N}} \text {, } \\
& \mathfrak{h}:(v \otimes v)=0 \\
& \text { on } \Sigma_{\mathrm{D}} \cup \Sigma_{\mathrm{N}} \text {, } \\
& \llbracket \mathbb{D} e(\dot{u})+\mathbb{C} e(u)-\mathbb{B} \Theta(\vartheta)-\operatorname{div} \mathfrak{h} \rrbracket v_{\mathrm{c}}-\operatorname{div}_{\mathrm{S}}\left(\llbracket \mathfrak{h} \rrbracket v_{\mathrm{c}}\right)=0 \\
& \mathfrak{h}^{+}:\left(v_{\mathrm{C}} \otimes v_{\mathrm{C}}\right)=\mathfrak{h}^{-}:\left(v_{\mathrm{C}} \otimes v_{\mathrm{C}}\right)=0 \\
& \partial I_{K}(\llbracket u \rrbracket)+\mathcal{J}\left(u, \dot{u}, \vartheta, v_{\mathrm{c}}\right)-\operatorname{div}_{\mathrm{S}}\left(\mathfrak{h} \cdot v_{\mathrm{C}}\right)+z \mathbb{A} \llbracket u \rrbracket-z a_{0}^{\prime}(\llbracket u \rrbracket) \ni 0 \\
& \partial I_{(-\infty, 0]}(\dot{z})+\partial I_{[0,1]}(z)+\frac{1}{2} \mathbb{A} \llbracket u \rrbracket \cdot \llbracket u \rrbracket-a_{0}(\llbracket u \rrbracket)-a_{1}(\llbracket u \rrbracket) \ni 0 \\
& \frac{1}{2}\left(\left.\mathcal{K}(e(u), \vartheta) \nabla \vartheta\right|_{\Gamma_{\mathrm{C}}} ^{+}+\left.\mathcal{K}(e(u), \vartheta) \nabla \vartheta\right|_{\Gamma_{\mathrm{C}}}\right) \cdot v_{\mathrm{C}}+\eta(\llbracket u \rrbracket, z) \llbracket \Theta(\vartheta) \rrbracket=0 \\
& \llbracket \mathcal{K}(e(u), \vartheta) \nabla \vartheta \rrbracket \cdot v_{\mathrm{C}}=-a_{1}(\llbracket u \rrbracket) \dot{z}
\end{aligned}
$$

with

$$
\begin{aligned}
\mathcal{J}(u, v, \vartheta, n): & =T(u, v, \Theta(\vartheta), n) \\
& =\left.[\mathbb{D} e(v)+\mathbb{C} e(u)-\mathbb{B} \Theta(\vartheta)-\operatorname{div}(\mathbb{H} \nabla e(u)+\mathbb{G} \nabla e(v))]\right|_{\Gamma^{n}}
\end{aligned}
$$

where again we take as $n$ the unit normal $v$ to $\Gamma_{\mathrm{N}}$ of $\Gamma=\Gamma_{\mathrm{N}}$, and $n=v_{\mathrm{C}}$ if $\Gamma=\Gamma_{\mathrm{C}}$.

REMARK 3.1 The reformulation of the heat equation (2.6b), viz. the second of (3.3a), shows the advantage of the enthalpy transformation (3.1). Indeed, by means of (3.1), the nonlinear term $c_{\mathrm{v}}(\theta) \dot{\theta}$ in (2.6b) has been replaced by the linear contribution $\dot{\vartheta}$. This makes the second of (3.3a) amenable to the time-discretization procedure developed in Section 5. Such a procedure would be more troublesome, if directly implemented on the heat equation (2.6b), also taking into account the growth conditions on the heat capacity function $c_{\mathrm{v}}$, which we shall impose in (4.1b) (for analogous assumptions, see [35, 37, 39], and [10, Sect.5.4.2] for contact problems in thermo-viscoelasticity). 
Functional setup. Throughout the paper, we shall extensively exploit that, in our 3-dimensional case,

$$
\begin{aligned}
& W^{2,2}(\Omega) \subset W^{1, p}(\Omega) \text { continuously for } 1 \leqslant p \leqslant 6, \text { and } \\
& \left.u \mapsto u\right|_{\Gamma}: W^{2,2}(\Omega) \rightarrow C(\Gamma) \text { compactly, }
\end{aligned}
$$

with $\Gamma=\partial \Omega$, or $\Gamma=\Gamma_{\mathrm{c}}$, or $\Gamma=\Gamma_{\mathrm{N}}$. Moreover, for $\gamma \in[2, \infty)$ we will adopt the notation

$$
W_{\Gamma_{\mathrm{D}}}^{2, \gamma}\left(\Omega \backslash \Gamma_{\mathrm{C}} ; \mathbb{R}^{3}\right):=\left\{v \in W^{2, \gamma}\left(\Omega \backslash \Gamma_{\mathrm{C}} ; \mathbb{R}^{3}\right): \quad v=0 \text { on } \Gamma_{\mathrm{D}}\right\},
$$

and denote by $\langle\cdot, \cdot\rangle\rangle$ the duality pairing between the spaces $W_{\Gamma_{\mathrm{D}}}^{2, \gamma}\left(\Omega \backslash \Gamma_{\mathrm{C}} ; \mathbb{R}^{3}\right)^{*}$ and $W_{\Gamma_{\mathrm{D}}}^{2, \gamma}\left(\Omega \backslash \Gamma_{\mathrm{C}} ; \mathbb{R}^{3}\right)$. Furthermore, in the case $K(x)$ is a linear subspace of $\mathbb{R}^{3}$ for almost all $x \in \Gamma_{\mathrm{C}}$, we shall use the notation

$$
W_{K}^{2,2}\left(\Omega \backslash \Gamma_{\mathrm{C}} ; \mathbb{R}^{3}\right):=\left\{v \in W_{\Gamma_{\mathrm{D}}}^{2,2}\left(\Omega \backslash \Gamma_{\mathrm{C}} ; \mathbb{R}^{3}\right): \llbracket v(x) \rrbracket \in K(x) \text { for a.a. } x \in \Gamma_{\mathrm{C}}\right\}
$$

We will work with the space of measures $m(\bar{\Omega}):=C(\bar{\Omega})^{*}$. Finally, let $X$ be a (separable) Banach space: we denote by $m([0, T] ; X), B_{\mathrm{w} *}([0, T] ; X), C_{\mathrm{w}}([0, T] ; X)$, and $B V([0, T] ; X)$, respectively, the Banach spaces of the measures on $[0, T]$ with values in $X$, of the functions from $[0, T]$ with values in $X$ that are bounded and weakly* measurable (if $X$ has a predual), of the functions from $[0, T]$ with values in $X$ that are weakly continuous, and of the functions that have bounded variation on $[0, T]$. Notice that the functions in $B_{\mathrm{w} *}([0, T] ; X)$ and $B V([0, T] ; X)$ are defined everywhere on $[0, T]$.

Loading qualification. Hereafter, the external mechanical and thermal loading $F, f$, and $g$ will be qualified by

$$
\begin{aligned}
& F \in L^{1}\left(0, T ; L^{2}\left(\Omega ; \mathbb{R}^{3}\right)\right), \\
& f \in W^{1,1}\left(0, T ; L^{4 / 3}\left(\Gamma_{\mathrm{N}} ; \mathbb{R}^{3}\right)\right), \\
& g \in L^{1}(\Sigma), \quad g \geqslant 0 \text { a.e. in } \Sigma .
\end{aligned}
$$

Initial data qualification. As for the initial data, we impose the following

$$
\begin{aligned}
& u_{0} \in W_{\Gamma_{\mathrm{D}}}^{2,2}\left(\Omega \backslash \Gamma_{\mathrm{c}} ; \mathbb{R}^{3}\right), \quad \llbracket u_{0} \rrbracket \succeq 0 \text { on } \Gamma_{\mathrm{C}}, \\
& \dot{u}_{0} \in L^{2}\left(\Omega ; \mathbb{R}^{3}\right) \quad \text { if } \varrho>0, \\
& z_{0} \in L^{\infty}\left(\Gamma_{\mathrm{c}}\right), \quad 0 \leqslant z_{0} \leqslant 1 \text { a.e. on } \Gamma_{\mathrm{C}}, \\
& \theta_{0} \in L^{\omega}(\Omega), \quad \theta_{0} \geqslant 0 \text { a.e. in } \Omega,
\end{aligned}
$$

where $\omega$ is as in (4.1b) below.

Weak formulation. The energetic formulation associated with system (3.3) hinges on the following energy functional $\Phi$, which is in fact the mechanical part of the free energy (2.13), and on the 
dissipation metric $R$ :

$$
\begin{aligned}
& \Phi(u, z):=\frac{1}{2} \int_{\Omega \backslash \Gamma_{\mathrm{C}}} \mathbb{C} e(u): e(u)+\mathbb{H} \nabla e(u): \nabla e(u) \mathrm{d} x+I_{K}(\llbracket u \rrbracket)+\int_{\Gamma_{\mathrm{C}}} z \alpha_{0}(\llbracket u \rrbracket)+I_{[0,1]}(z) \mathrm{d} S, \\
& R(u, \dot{z}):= \begin{cases}\int_{\Gamma_{\mathrm{C}}} a_{1}(\llbracket u \rrbracket)|\dot{z}| \mathrm{d} S & \text { if } \dot{z} \leqslant 0 \text { a.e. in } \Gamma_{\mathrm{C}}, \\
+\infty & \text { otherwise. }\end{cases}
\end{aligned}
$$

We are now in the position of introducing the notion of weak solution to system (3.3) which shall be analyzed throughout this paper.

DEFINITION 3.2 (Energetic solution of the adhesive contact problem) Given a quadruple of initial data $\left(u_{0}, \dot{u}_{0}, z_{0}, \theta_{0}\right)$ satisfying conditions (3.7), we call a triple $(u, z, \vartheta)$ an energetic solution to the Cauchy problem for (the enthalpy reformulation of) system (3.3) if

$u \in W^{1,2}\left(0, T ; W_{\Gamma_{\mathrm{D}}}^{2,2}\left(\Omega \backslash \Gamma_{\mathrm{C}} ; \mathbb{R}^{3}\right)\right)$,

$u \in W^{1, \infty}\left(0, T ; L^{2}\left(\Omega ; \mathbb{R}^{3}\right)\right)$ with $\dot{u} \in C_{\mathrm{w}}\left([0, T] ; L^{2}\left(\Omega ; \mathbb{R}^{3}\right)\right) \quad$ if $\varrho>0$,

$z \in L^{\infty}\left(\Sigma_{\mathrm{c}}\right) \cap B V\left([0, T] ; L^{1}\left(\Gamma_{\mathrm{c}}\right)\right), z(\cdot, x)$ nonincreasing on $[0, T]$ for a.a. $x \in \Gamma_{\mathrm{C}}$,

$\left.\begin{array}{l}\vartheta \in L^{r}\left(0, T ; W^{1, r}\left(\Omega \backslash \Gamma_{\mathrm{C}}\right)\right) \cap L^{\infty}\left(0, T ; L^{1}(\Omega)\right) \cap B_{\mathrm{w} *}([0, T] ; m(\bar{\Omega})), \\ \vartheta \in B V\left([0, T] ; W^{1, r^{\prime}}\left(\Omega \backslash \Gamma_{\mathrm{C}}\right)^{*}\right)\end{array}\right\}$ for any $1 \leqslant r<\frac{5}{4}$,

with $r^{\prime}$ denoting the conjugate exponent $\frac{r}{r-1}$ of $r$, and the triple $(u, z, \vartheta)$ complies with:

(i) (weak formulation of the) momentum inclusion, i.e.:

$$
\begin{aligned}
& \llbracket u \rrbracket \succeq 0 \text { on } \Sigma_{\mathrm{C}}, \quad \text { and } \\
& \qquad \int_{\Omega} \dot{u}(T) \cdot(v(T)-u(T)) \mathrm{d} x+\int_{Q}(\mathbb{D} e(\dot{u})+\mathbb{C} e(u)-\mathbb{B} \Theta(\vartheta)): e(v-u)-\varrho \dot{u} \cdot(\dot{v}-\dot{u}) \\
& \left.\left.\quad+(\mathbb{H} \nabla e(u)+\mathbb{G} \nabla e(\dot{u})) \vdots \nabla e(v-u) \mathrm{d} x \mathrm{~d} t+\int_{\Sigma_{\mathrm{C}}} z \alpha_{0}^{\prime}(\llbracket u]\right) \cdot \llbracket v-u\right] \mathrm{d} S \mathrm{~d} t \\
& \quad \geqslant \varrho \int_{\Omega} \dot{u}_{0} \cdot(v(0)-u(0)) \mathrm{d} x+\int_{Q} F \cdot(v-u) \mathrm{d} x \mathrm{~d} t+\int_{\Sigma_{\mathrm{N}}} f \cdot(v-u) \mathrm{d} S \mathrm{~d} t
\end{aligned}
$$

for all $v$ in $L^{2}\left(0, T ; W_{\Gamma_{\mathrm{D}}}^{2,2}\left(\Omega \backslash \Gamma_{\mathrm{C}} ; \mathbb{R}^{3}\right)\right)$ with $\llbracket v \rrbracket \geq 0$ on $\Sigma_{\mathrm{C}}$ and, if $\varrho>0$, also in $W^{1,1}\left(0, T ; L^{2}\left(\Omega ; \mathbb{R}^{3}\right)\right)$,

(ii) total energy balance

$$
\begin{array}{r}
T_{\text {kin }}(\dot{u}(T))+\Phi(u(T), z(T))+\int_{\Omega} \vartheta(T)(\mathrm{d} x)=T_{\text {kin }}\left(\dot{u}_{0}\right)+\Phi\left(u_{0}, z_{0}\right)+\int_{\Omega} \vartheta_{0} \mathrm{~d} x \\
+\int_{Q} F \cdot \dot{u} \mathrm{~d} x \mathrm{~d} t+\int_{\Sigma_{\mathrm{N}}} f \cdot \dot{u} \mathrm{~d} S \mathrm{~d} t+\int_{\Sigma} g \mathrm{~d} S \mathrm{~d} t
\end{array}
$$


(iii) semistability for a.a. $t \in(0, T)$

$$
\forall \tilde{z} \in L^{\infty}\left(\Gamma_{\mathrm{C}}\right): \quad \Phi(u(t), z(t)) \leqslant \Phi(u(t), \tilde{z})+\mathbb{R}(u(t), \tilde{z}-z(t)),
$$

(iv) (weak formulation of the) enthalpy equation:

$$
\begin{aligned}
& \left.\int_{\bar{\Omega}} w(T) \vartheta(T)(\mathrm{d} x)+\int_{Q} \mathcal{K}(e(u), \vartheta) \nabla \vartheta \cdot \nabla w-\vartheta \dot{w} \mathrm{~d} x \mathrm{~d} t+\int_{\Sigma_{\mathrm{C}}} \eta(\llbracket u \rrbracket, z) \llbracket \Theta(\vartheta)\right] \llbracket w \rrbracket \mathrm{d} S \mathrm{~d} t \\
& =\int_{Q}(\mathbb{D} e(\dot{u}): e(\dot{u})-\Theta(\vartheta) \mathbb{B}: e(\dot{u})+\mathbb{G} \nabla e(\dot{u}): \nabla e(\dot{u})) w \mathrm{~d} x \mathrm{~d} t \\
& \quad-\int_{\bar{\Sigma}_{\mathrm{C}}} \frac{\left.w\right|_{\Gamma_{\mathrm{C}}} ^{+}+\left.w\right|_{\Gamma_{\mathrm{C}}} ^{-}}{2} a_{1}(\llbracket u \rrbracket) \dot{z}(\mathrm{~d} S \mathrm{~d} t)+\int_{\Sigma} g w \mathrm{~d} S \mathrm{~d} t+\int_{\Omega} \vartheta_{0} w(0) \mathrm{d} x
\end{aligned}
$$

for all $w \in C\left([0, T] ; W^{1, r^{\prime}}\left(\Omega \backslash \Gamma_{\mathrm{C}}\right)\right) \cap W^{1, r^{\prime}}\left(0, T ; L^{r^{\prime}}(\Omega)\right)$, where $\vartheta_{0}:=h_{0}\left(\theta_{0}\right)$, and $\vartheta(T)$ and $\dot{z}$ are considered as measures on $\bar{\Omega}$ and $\bar{\Sigma}_{\mathrm{C}}$, respectively,

(v) and the remaining initial conditions (beside $\dot{u}(0)=\dot{u}_{0}$, which is already enforced in (3.11b)), i.e.

$$
u(0)=u_{0} \quad \text { a.e. in } \Omega, \quad z(0)=z_{0} \quad \text { a.e. in } \Gamma_{\mathrm{c}}, \quad \vartheta(0)=\vartheta_{0} \quad \text { a.e. in } \Omega .
$$

REMARK 3.3 (The weak formulation (3.11b) of the momentum inclusion) In order to (partially) justify (3.11b) and its link with the classical formulation (2.6a,c,d,f-k) of the (boundary-value problem for the) momentum inclusion, we may observe that, upon multiplying (2.6a) by $v-u$ (with $v$ an admissible test function in the sense of Definition 3.2) and integrating on $Q$, one has to deal with the term

$$
-\int_{Q} \operatorname{div} \sigma \cdot(v-u) \mathrm{d} x \mathrm{~d} t=-\int_{0}^{T} \int_{\Omega} \operatorname{div} \sigma_{\mathrm{KV}} \cdot(v-u) \mathrm{d} x \mathrm{~d} t+\int_{0}^{T} \int_{\Omega} \operatorname{div}^{2} \mathfrak{h} \cdot(v-u) \mathrm{d} x \mathrm{~d} t,
$$

where $\sigma_{\mathrm{KV}}$ is a placeholder for the "Kelvin-Voigt" stress $\mathbb{D} e(\dot{u})+\mathbb{C} e(u)-\mathbb{B} \theta$. The treatment of the first integral term on the right-hand side involves a standard integration by parts. As for the second one, let us observe (neglecting time-integration and integrating by parts twice, with the zero Dirichlet condition on $\Gamma_{\mathrm{D}}$ ), that

$$
\begin{aligned}
\int_{\Omega} \operatorname{div}^{2} \mathfrak{h} \cdot(v-u) \mathrm{d} x=- & \int_{\Omega_{+}}(\operatorname{divh}): \nabla(v-u) \mathrm{d} x-\int_{\Omega_{-}}(\operatorname{divh}): \nabla(v-u) \mathrm{d} x \\
& +\int_{\Gamma_{\mathrm{N}}}(\operatorname{divh}):((v-u) \otimes v) \mathrm{d} S+\int_{\Gamma_{\mathrm{C}}}(\operatorname{divh})^{+}:\left(\left(v^{+}-u^{+}\right) \otimes v_{\mathrm{C}}\right) \mathrm{d} S \\
& \quad-\int_{\Gamma_{\mathrm{C}}}(\operatorname{divh})^{-}:\left(\left(v^{-}-u^{-}\right) \otimes v_{\mathrm{C}}\right) \mathrm{d} S \\
= & \int_{\Omega_{+}} \mathfrak{h}: \nabla^{2}(v-u) \mathrm{d} x+\int_{\Omega_{-}} \mathfrak{h}: \nabla^{2}(v-u) \mathrm{d} x \\
& +I\left(\Gamma_{\mathrm{N}}, u, v, v\right)+I\left(\Gamma_{\mathrm{C}}, u^{+}, v^{+}, v_{\mathrm{C}}\right)-I\left(\Gamma_{\mathrm{C}}, u^{-}, v^{-}, v_{\mathrm{C}}\right)
\end{aligned}
$$


where we have used the short-hand notation $I(\tilde{\Gamma}, \tilde{u}, \tilde{v}, \tilde{v}):=\int_{\tilde{\Gamma}}(\operatorname{divh}):((\tilde{v}-\tilde{u}) \otimes \tilde{v})-\mathfrak{h}:(\nabla(\tilde{v}-\tilde{u}) \otimes$ $\tilde{v}) \mathrm{d} S$. Then, the calculations developed in [36, 2nd ed., Sect.2.4.4], [40] and based on the decomposition $\nabla v=\nabla_{\mathrm{s}} v+\frac{\partial v}{\partial n} n$ yield the following formula

$$
I(\tilde{\Gamma}, \tilde{u}, \tilde{v}, \tilde{v})=\int_{\tilde{\Gamma}}\left((\operatorname{divh}) \cdot \tilde{v}+\operatorname{div}_{\mathrm{S}}(\mathfrak{h} \cdot \tilde{v})-\left(\operatorname{div}_{\mathrm{S}} \tilde{v}\right)(\mathfrak{h}:(\tilde{v} \otimes \tilde{v}))\right) \cdot(\tilde{v}-\tilde{u})-(\mathfrak{h}:(\tilde{v} \otimes \tilde{v})) \cdot \frac{\partial(\tilde{v}-\tilde{u})}{\partial \tilde{v}} \mathrm{~d} S,
$$

which we plug in (3.12). Then, we combine the resulting integrals on $\Gamma_{\mathrm{N}}$ and $\Gamma_{\mathrm{C}}$ with the integrals derived from the by-part integration of $\int_{\Omega} \operatorname{div} \sigma_{\mathrm{KV}} \cdot(v-u) \mathrm{d} x$, rely on the boundary conditions (2.6d,fk), take into account the enthalpy transformation (3.1), and finally use that $\operatorname{div}^{2} \mathfrak{h} \cdot(v-u)=$ $\mathfrak{h}: \nabla e(v-u)$, since $\mathfrak{h}$ is symmetric, being so $\mathbb{G}$ and $\mathbb{H}$. In this way, we obtain the second and the third term on the left-hand side of $(3.11 \mathrm{~b})$. The remaining terms either follow from an integration by parts in time, or are trivial.

REMARK 3.4 (The "weak" formulation of the flow rule (2.10)) In [24, 27, 29], a global stability condition combined with energy conservation was shown to provide the correct "weak" formulation of rate-independent flow rules [24, 27-29]. Here, the concept of energy-preserving solutions (i.e., of energetic solutions) is crucial for mathematically treating the full thermodynamics, cf. Step 5 in Section 6 below. We point out that (3.11c) is the integrated version of the total energy balance (2.20). Here the energy conservation involves also the mechanical equilibrium (3.11b), and the semistability (3.11d) plays the role of the global stability condition of [24, 27, 29]. We refer to [39, Prop. 3.2] for some justification of the energetic-solution concept of Definition 3.2 in the framework of general thermodynamical rate-independent processes. In general, energetic solutions may exhibit unphysical jumps, but this does not occur if the driving energy $\Phi(u, \cdot)$ is convex, as it is indeed the case considered here, see (3.8). Then there is also a close link to the conventional weak definition of the flow rule (2.10), see [29].

REMARK 3.5 (The weak formulation (3.11e) of the enthalpy equation) A few comments on the first term on the left-hand side and on the second term on the right-hand side of (3.11e) are in order. First, since $\vartheta \in B V\left([0, T] ; W^{1, r^{\prime}}\left(\Omega \backslash \Gamma_{\mathrm{C}}\right)^{*}\right)$, then for all $t \in[0, T]$ one has $\vartheta(t)$ well-defined as an element of $W^{1, r^{\prime}}\left(\Omega \backslash \Gamma_{\mathrm{C}}\right)^{*}$. Combining this with the fact that $\vartheta \in L^{\infty}\left(0, T ; L^{1}(\Omega)\right)$ one sees that even $\vartheta(t) \in m(\bar{\Omega})$ for all $t \in[0, T]$. However, note that the function $t \mapsto \vartheta(t)$ may jump. Second, let us observe that due to (3.10c), $\dot{z}$ is a negative Radon measure on $\bar{\Sigma}_{\mathrm{C}}$. Since we shall impose that the function $a_{1}: \mathbb{R}^{3} \rightarrow \mathbb{R}$ is continuous (cf. (4.1h)), and since the map $(t, x) \mapsto \llbracket u(t, x) \rrbracket$ is also continuous because of (3.10a) and (3.4), it turns out that $(t, x) \mapsto a_{1}(\llbracket u(t, x) \rrbracket)$ is a continuous function. Thus, $a_{1}(\llbracket u \rrbracket) \dot{z}$ is a well-defined measure on $\bar{\Sigma}_{\mathrm{C}}$.

REMARK 3.6 (Mechanical energy equality) Subtracting (3.11e) tested by 1 from (3.11c) reveals that energetic solutions comply with the mechanical energy equality:

$$
\begin{aligned}
T_{\text {kin }}^{\varrho}(\dot{u}(T))+ & \Phi(u(T), z(T))+\int_{Q}(\mathbb{D} e(\dot{u}): e(\dot{u})+\mathbb{G} \nabla e(\dot{u}): \nabla e(\dot{u})) \mathrm{d} x \mathrm{~d} t+\operatorname{Var}_{\mathbb{R}}(u, z ;[0, T]) \\
& =T_{\text {kin }}^{\varrho}\left(\dot{u}_{0}\right)+\Phi\left(u_{0}, z_{0}\right)+\int_{Q} F \cdot \dot{u}+\Theta(\vartheta) \mathbb{B}: e(\dot{u}) \mathrm{d} x \mathrm{~d} t+\int_{\Sigma_{\mathrm{N}}} f \cdot \dot{u} \mathrm{~d} S \mathrm{~d} t, \quad
\end{aligned}
$$

where we have used the notation

$$
\operatorname{Var}_{\mathbb{R}}\left(u, z ;\left[t_{1}, t_{2}\right]\right):=\int_{t_{1}}^{t_{2}} \int_{\bar{\Gamma}_{\mathrm{C}}} a_{1}(\llbracket u \rrbracket)|\dot{z}|(\mathrm{d} S \mathrm{~d} t) \quad \text { for }\left[t_{1}, t_{2}\right] \subset[0, T] .
$$




\section{Main result}

We now enlist our conditions on the functions $c_{\mathrm{v}}, \mathbb{K}, \eta, a_{0}, a_{1}$, and the loading.

Assumptions. We suppose that

$$
\begin{aligned}
& c_{\mathrm{v}}:[0,+\infty) \rightarrow(0,+\infty) \text { continuous, } \\
& \exists \omega_{1} \geqslant \omega>\frac{6}{5}, c_{1} \geqslant c_{0}>0 \forall \theta \in(0,+\infty): \quad c_{0}(1+\theta)^{\omega-1} \leqslant c_{\mathrm{v}}(\theta) \leqslant c_{1}(1+\theta)^{\omega_{1}-1}, \\
& \mathbb{K}: \mathbb{R}^{3 \times 3} \times \mathbb{R} \rightarrow \mathbb{R}^{3 \times 3} \text { is bounded, continuous, and } \\
& \underset{(e, \vartheta, \xi) \in \mathbb{R}_{\mathrm{sym}}^{3 \times 3} \times \mathbb{R}^{3} \times \mathbb{R}^{3},|\xi|=1}{\mathcal{K}(e, \vartheta) \xi: \xi=: \mathrm{k}>0 .}
\end{aligned}
$$

We also require that $\eta(x, v, \cdot)$ is a non-negative affine function of the delamination parameter $z \in$ $[0,1]$, and, following [35], we assume that

$$
\begin{array}{r}
\eta(x, v, z)=\eta_{1}(x, v) z+\eta_{0}(x, v) \text { for } \eta_{1}, \eta_{0}: \Gamma_{\mathrm{C}} \times \mathbb{R}^{3} \rightarrow[0,+\infty) \text { Carathéodory s.t. } \\
\exists C_{\eta}>0 \quad \forall(x, v) \in \Gamma_{\mathrm{C}} \times \mathbb{R}^{3}:\left|\eta_{0}(x, v)\right|+\left|\eta_{1}(x, v)\right| \leqslant C_{\eta}\left(|v|^{4 / 3}+1\right) ;
\end{array}
$$

As for the functions $a_{0}: \mathbb{R}^{3} \rightarrow \mathbb{R}$ and $a_{1}: \mathbb{R}^{3} \rightarrow \mathbb{R}$, we suppose that

$$
\begin{aligned}
& a_{0} \in C^{1}\left(\mathbb{R}^{3} ; \mathbb{R}\right), \quad \exists C_{a_{0}}, C_{a_{0}}^{\prime}>0 \forall v \in \mathbb{R}^{3}: \quad\left|a_{0}(v)\right| \leqslant C_{a_{0}}|v|+C_{a_{0}}^{\prime}, \\
& \text { the map } u \mapsto \frac{1}{2} \mathbb{A} u \cdot u-a_{0}(u)=: \alpha_{0}(u) \text { is convex, } \\
& a_{1} \in C\left(\mathbb{R}^{3} ; \mathbb{R}\right), \quad \exists C_{a_{1}}>0 \forall v \in \mathbb{R}^{3}: \quad a_{1}(v) \geqslant C_{a_{1}}>0 .
\end{aligned}
$$

REMARK 4.1 Let us comment on conditions (4.1). First of all, it is immediate to deduce from (4.1b) that

$$
\exists C_{\theta}^{1}, C_{\theta}^{2}>0 \forall w \in[0,+\infty):\left(C_{\theta}^{1} w+1\right)^{1 / \omega_{1}}-1 \leqslant \Theta(w) \leqslant\left(C_{\theta}^{2} w+1\right)^{1 / \omega}-1 .
$$

In fact, the slight growth condition (4.1b) for $c_{\mathrm{v}}$ is needed for the interpolation estimate (5.28), which in turn is taken from [37, 39] on models for simple materials. To weaken $(4.1 \mathrm{~b})$, one might impose the heat conductivity $\mathbb{K}$ to depend on $\nabla \theta$ with a certain growth, cf. [10]. Alternatively, one might use a finer interpolation exploiting the higher terms of the nonsimple materials as devised in [40]. However, this would make the basic energetics essentially dependent on these higher order terms, which perhaps would not be much physical. Furthermore, it obviously follows from (4.1c) that

$$
\exists C_{\mathcal{K}}>0 \quad \forall \xi, \zeta \in \mathbb{R}^{3}: \quad|\mathcal{K}(e, \vartheta) \xi: \zeta| \leqslant C_{\mathcal{K}}|\xi||\zeta| .
$$

Moreover, let us observe that the functional $u \mapsto \Phi(u, z)$ is convex thanks to (4.1g). Note that $a_{0}$ itself need not be concave, and the possible violation of concavity depends on the positivedefiniteness of $\mathbb{A}$. Actually, we could even allow for bigger violation (namely for $a_{0}$ semi-concave), if the discretization scheme were slightly modified, like for example in [36, 2nd ed., Rem. 8.2.4]. However, we have chosen not to explore this option, since in real-world applications $\mathbb{A}$ is large.

THEOREM 4.2 (Existence for the adhesive contact problem) Let us assume (3.6), (4.1), (3.7) and (i) if $\varrho=0$, suppose also

$$
\begin{aligned}
& F \in W^{1,1}\left(0, T ; L^{6 / 5}\left(\Omega ; \mathbb{R}^{3}\right)\right), \\
& \mathscr{H}^{2}\left(\partial \Omega_{+} \cap \Gamma_{\mathrm{D}}\right)>0, \quad \mathscr{H}^{2}\left(\partial \Omega_{-} \cap \Gamma_{\mathrm{D}}\right)>0,
\end{aligned}
$$


where $\mathscr{H}^{2}$ denotes the two-dimensional Hausdorff measure, and

(ii) if $\varrho>0$, suppose also that

$$
K(x) \text { is a linear subspace of } \mathbb{R}^{3} \text { for a.a. } x \in \Gamma_{\mathrm{c}} .
$$

Then, there exists an energetic solution $(u, z, \vartheta)$ to the adhesive contact problem (in the sense of Definition 3.2), with the additional regularity

$$
\dot{u} \in W^{1,2}\left(0, T ; W_{K}^{2,2}\left(\Omega \backslash \Gamma_{\mathrm{C}} ; \mathbb{R}^{3}\right)^{*}\right) \quad \text { if } \varrho>0 .
$$

Furthermore, in both cases $\varrho>0$ and $\varrho=0$, the positivity of the initial temperature

$$
\inf _{x \in \Omega} \theta_{0}=: \theta^{*}>0
$$

implies $\inf _{(t, x) \in Q} \theta=\inf _{(t, x) \in Q} \Theta(\vartheta(t, x))>0$; in particular, $\theta$ is a.e. positive on $Q$.

Let us observe that $\dot{u} \in L^{\infty}\left(0, T ; L^{2}\left(\Omega ; \mathbb{R}^{3}\right)\right) \cap W^{1,2}\left(0, T ; W_{K}^{2,2}\left(\Omega \backslash \Gamma_{\mathrm{C}} ; \mathbb{R}^{3}\right)^{*}\right)$ yields also that $\dot{u} \in C_{\mathrm{w}}\left([0, T] ; L^{2}\left(\Omega ; \mathbb{R}^{3}\right)\right)$ as required in $(3.10 \mathrm{~b})$, so that $\dot{u}(T)$ in $(3.11 \mathrm{~b}, \mathrm{c})$ makes sense.

REMARK 4.3 The analytical reason why in the presence of inertial terms in the momentum equation we need to restrict to "linear" contact conditions on $\Gamma_{\mathrm{c}}$ is ultimately that, if $\varrho>0$, only (4.5) makes it possible to test the (weak formulation of the) momentum equation by the velocity $\dot{u}$. This is needed for obtaining the mechanical energy equality (3.13), which in turn is a crucial step in the proof of Theorem 4.2.

Indeed, the analysis of the momentum equilibrium equation in which inertia interacts with Signorini boundary conditions is remarkably difficult. It has indeed been an open problem for a long time. In this connection, we may mention the recent results obtained in [30] for the (uncoupled) dynamical viscoelastic equation with Signorini contact conditions in the one- and three-dimensional case on unbounded domains. In such a context, these existence results have been proved with refined Fourier analysis techniques.

In what follows, we shall denote by the symbols $C, C^{\prime}$ most of the (positive) constants occurring in calculations and estimates.

\section{Semi-implicit time discretization}

We perform a semi-implicit time-discretization using an equidistant partition of $[0, T]$, with timestep $\tau>0$ and nodes $t_{\tau}^{k}:=k \tau, k=0, \ldots, K_{\tau}$. Hereafter, given any sequence $\left\{\phi^{j}\right\}_{j \geqslant 1}$, we will denote the backward difference operator and its iteration by, respectively,

$$
\mathrm{D}_{t} \phi^{k}:=\frac{\phi^{k}-\phi^{k-1}}{\tau}, \quad \mathrm{D}_{t}^{2} \phi^{k}:=\mathrm{D}_{t}\left(\mathrm{D}_{t} \phi^{k}\right)=\frac{\phi^{k}-2 \phi^{k-1}+\phi^{k-2}}{\tau^{2}} .
$$

We approximate the data $F, f$ by local means, i.e. setting for all $k=1, \ldots, K_{\tau}$

$$
F_{\tau}^{k}:=\frac{1}{\tau} \int_{t_{\tau}^{k-1}}^{t_{\tau}^{k}} F(s) \mathrm{d} s, \quad f_{\tau}^{k}:=\frac{1}{\tau} \int_{t_{\tau}^{k-1}}^{t_{\tau}^{k}} f(s) \mathrm{d} s
$$


Furthermore, we approximate $g$ by suitably constructed discrete data $\left\{g_{\tau}^{k}\right\}_{k=1}^{K_{\tau}} \subset H^{1 / 2}(\partial \Omega)^{*}$ such that (5.9) below holds, and the initial datum $u_{0}$ by a sequence $\left\{u_{0, \tau}\right\} \subset W_{\Gamma_{\mathrm{D}}}^{2, \gamma}\left(\Omega \backslash \Gamma_{\mathrm{C}} ; \mathbb{R}^{3}\right)$ (with $\gamma>\max \left\{4, \frac{2 \omega}{\omega-1}\right\}$ as assumed in Problem 5.1) such that

$$
\lim _{\tau \downarrow 0} \tau^{1 / \gamma}\left\|\nabla e\left(u_{0, \tau}\right)\right\|_{L^{\gamma}\left(\Omega ; \mathbb{R}^{3 \times 3 \times 3}\right)}=0, \quad u_{0, \tau} \rightarrow u_{0} \quad \text { in } W^{2,2}\left(\Omega ; \mathbb{R}^{3}\right) \text { as } \tau \rightarrow 0 .
$$

We are now in the position of formulating the time-discrete problem, which we again write in the classical formulation for notational simplicity. While referring to the forthcoming Remark 5.2 for all details, we may mention in advance that in Problem 5.1 a careful choice of the terms to be kept implicit vs. explicit has been made in order to guarantee positivity of the (discrete) temperature $\vartheta_{\tau}^{k}$ on the one hand, and some simplifications in the a priori estimates on the other hand. Furthermore, a regularizing term has been added to the momentum equation.

Problem 5.1 Let $\gamma>\max \left\{4, \frac{2 \omega}{\omega-1}\right\}$. Given

$$
u_{\tau}^{0}=u_{0, \tau}, \quad u_{\tau}^{-1}=u_{0, \tau}-\tau \dot{u}_{0}, \quad z_{\tau}^{0}=z_{0}, \quad \vartheta_{\tau}^{0}=\vartheta_{0},
$$

find $\left\{\left(u_{\tau}^{k}, \vartheta_{\tau}^{k}, z_{\tau}^{k}\right)\right\}_{k=1}^{K_{\tau}}$ fulfilling, for $k=1, \ldots, K_{\tau}$, the recursive scheme consisting of the discrete momentum equation in $\Omega \backslash \Gamma_{\mathrm{C}}$ :

$$
\left.\begin{array}{c}
\varrho \mathrm{D}_{t}^{2} u_{\tau}^{k}-\operatorname{div}\left(\mathbb{D} e\left(\mathrm{D}_{t} u_{\tau}^{k}\right)+\mathbb{C} e\left(u_{\tau}^{k}\right)-\mathbb{B} \Theta\left(\vartheta_{\tau}^{k}\right)+\tau\left|e\left(u_{\tau}^{k}\right)\right|^{\gamma-2} e\left(u_{\tau}^{k}\right)-\operatorname{divh} \mathfrak{h}_{\tau}^{k}\right) \\
=F_{\tau}^{k} \quad \text { with } \quad \mathfrak{h}_{\tau}^{k}=\left(\mathbb{H}+\tau\left|\nabla e\left(u_{\tau}^{k}\right)\right|^{\gamma-2} \mathbb{I}\right) \nabla e\left(u_{\tau}^{k}\right)+\mathbb{G} \nabla\left(e\left(\mathrm{D}_{t} u_{\tau}^{k}\right)\right)
\end{array}\right\} \text { in } \Omega \backslash \Gamma_{\mathrm{C}},
$$

where $\mathbb{I}: \mathbb{R}^{3 \times 3 \times 3} \rightarrow \mathbb{R}^{3 \times 3 \times 3}$ denotes the 6th-order identity tensor, with the boundary conditions

$$
\begin{array}{ll}
u_{\tau}^{k}=0 & \text { on } \Gamma_{\mathrm{D}}, \\
\left(\mathbb{D} e\left(\mathrm{D}_{t} u_{\tau}^{k}\right)+\mathbb{C} e\left(u_{\tau}^{k}\right)-\Theta\left(\vartheta_{\tau}^{k}\right) \mathbb{B}\right. & \\
\left.\quad+\tau\left|e\left(u_{\tau}^{k}\right)\right|^{\gamma-2} e\left(u_{\tau}^{k}\right)-\operatorname{div} \mathfrak{h}_{\tau}^{k}\right) v-\operatorname{div}_{\mathrm{S}}\left(\mathfrak{h}_{\tau}^{k} \cdot v\right)=f_{\tau}^{k} & \text { on } \Gamma_{\mathrm{N}}, \\
\mathfrak{h}_{\tau}^{k}:(v \otimes v)=0 & \text { on } \Gamma_{\mathrm{D}} \cup \Gamma_{\mathrm{N}},
\end{array}
$$

and the conditions on the contact boundary

$$
\left.\begin{array}{rl}
\llbracket \mathbb{D} e\left(\mathrm{D}_{t} u_{\tau}^{k}\right)+\mathbb{C} e\left(u_{\tau}^{k}\right)-\Theta\left(\vartheta_{\tau}^{k}\right) \mathbb{B}+\tau\left|e\left(u_{\tau}^{k}\right)\right|^{\gamma-2} e\left(u_{\tau}^{k}\right)-\operatorname{div}\left(\mathfrak{h}_{\tau}^{k}\right) \rrbracket v_{\mathrm{c}} \\
\left(\mathfrak{h}_{\tau}^{k}\right)^{+}:\left(v_{\mathrm{C}} \otimes v_{\mathrm{C}}\right)=\left(\mathfrak{h}_{\tau}^{k}\right)^{-}:\left(v_{\mathrm{c}} \otimes v_{\mathrm{c}}\right)=0, & -\operatorname{div}_{\mathrm{S}}\left(\llbracket \mathfrak{h}_{\tau}^{k} \rrbracket v_{\mathrm{c}}\right)=0, \\
z_{\tau}^{k-1} \alpha_{0}^{\prime}\left(\llbracket u_{\tau}^{k} \rrbracket\right)+ & \partial I_{K}\left(\llbracket u_{\tau}^{k} \rrbracket\right)+\left(\mathbb{D} e\left(\mathrm{D}_{t} u_{\tau}^{k}\right)+\mathbb{C} e\left(u_{\tau}^{k}\right)-\Theta\left(\vartheta_{\tau}^{k}\right) \mathbb{B}\right. \\
& \left.+\tau\left|e\left(u_{\tau}^{k}\right)\right|^{\gamma-2} e\left(u_{\tau}^{k}\right)-\operatorname{divh}_{\tau}^{k}\right) v_{\mathrm{c}}-\operatorname{div}_{\mathrm{S}}\left(\mathfrak{h}_{\tau}^{k} \cdot v_{\mathrm{c}}\right)=0,
\end{array}\right\} \text { on } \Gamma_{\mathrm{C}} ;
$$

further, the discrete enthalpy equation:

$$
\begin{aligned}
\mathrm{D}_{t} \vartheta_{\tau}^{k}-\operatorname{div}\left(\mathcal{K}\left(\vartheta_{\tau}^{k}, e\left(u_{\tau}^{k}\right)\right) \nabla \vartheta_{\tau}^{k}\right)=\mathbb{D} e\left(\mathrm{D}_{t} u_{\tau}^{k}\right): e\left(\mathrm{D}_{t} u_{\tau}^{k}\right) \\
-\Theta\left(\vartheta_{\tau}^{k}\right) \mathbb{B}: e\left(\mathrm{D}_{t} u_{\tau}^{k}\right)+\mathbb{G} \nabla e\left(\mathrm{D}_{t} u_{\tau}^{k}\right) \vdots \nabla e\left(\mathrm{D}_{t} u_{\tau}^{k}\right) \quad \text { in } \Omega \backslash \Gamma_{\mathrm{C}},
\end{aligned}
$$


with the boundary conditions

$$
\left(\mathcal{K}\left(\vartheta_{\tau}^{k}, e\left(u_{\tau}^{k}\right)\right) \nabla \vartheta_{\tau}^{k}\right) \cdot v=g_{\tau}^{k} \quad \text { on } \Gamma_{\mathrm{D}} \cup \Gamma_{\mathrm{N}}
$$

and the conditions on the contact boundary

$$
\left.\begin{array}{l}
\frac{1}{2}\left(\left.\mathcal{K}\left(\vartheta_{\tau}^{k}, e\left(u_{\tau}^{k}\right)\right) \nabla \vartheta_{\tau}^{k}\right|_{\Gamma_{\mathrm{C}}} ^{+}+\left.\mathcal{K}\left(\vartheta_{\tau}^{k}, e\left(u_{\tau}^{k}\right)\right) \nabla \vartheta_{\tau}^{k}\right|_{\Gamma_{\mathrm{C}}} ^{-}\right) \cdot v_{\mathrm{C}} \\
+\eta\left(\llbracket u_{\tau}^{k-1} \rrbracket, z_{\tau}^{k}\right) \llbracket \Theta\left(\vartheta_{\tau}^{k}\right) \rrbracket=0, \\
\llbracket \mathcal{K}\left(\vartheta_{\tau}^{k}, e\left(u_{\tau}^{k}\right)\right) \nabla \vartheta_{\tau}^{k} \rrbracket v_{\mathrm{C}}=-a_{1}\left(\llbracket u_{\tau}^{k} \rrbracket\right) \mathrm{D}_{t} z_{\tau}^{k}
\end{array}\right\} \text { on } \Gamma_{\mathrm{C}} ;
$$

and also the discrete flow rule for the delamination parameter

$$
\partial_{v} \mathcal{F}\left(z_{\tau}^{k-1} ; z_{\tau}^{k}\right)+\alpha_{0}\left(\llbracket u_{\tau}^{k} \rrbracket\right)-a_{1}\left(\llbracket u_{\tau}^{k} \rrbracket\right) \ni 0 \text { on } \Gamma_{\mathrm{C}},
$$

where $\mathcal{F}\left(z_{\tau}^{k-1} ; \cdot\right): \mathbb{R} \rightarrow[0,+\infty]$ is the convex functional

$$
\mathcal{F}\left(z_{\tau}^{k-1} ; v\right)=I_{(-\infty, 0]}\left(\frac{v-z_{\tau}^{k-1}}{\tau}\right)+I_{[0,1]}(v) .
$$

In the last condition in (5.4e), traces of the overall stress either from $\Omega_{+}$or from $\Omega_{-}$can be considered with the same effect, thanks to the first boundary condition in $(5.4 \mathrm{e})$.

REMARK 5.2 Let us highlight the main features of the time-discrete scheme (5.4).

First, the discrete version (5.4f) of the enthalpy equation is fully implicit, and in particular on the right-hand side the term $\Theta\left(\vartheta_{\tau}^{k}\right) \mathbb{B}: e\left(\mathrm{D}_{t} u_{\tau}^{k}\right)$ appears, instead of $\Theta\left(\vartheta_{\tau}^{k-1}\right) \mathbb{B}: e\left(\mathrm{D}_{t} u_{\tau}^{k}\right)$. This is crucial to obtain the positivity of the temperature, i.e., $\vartheta_{\tau}^{k} \geqslant 0$ a.e. in $\Omega$, cf. Lemma 5.4. Notice that the term $\mathbb{B} \Theta\left(\vartheta_{\tau}^{k}\right)$ occurs on the left-hand side of (5.4a): therefore, (5.4a) and (5.4f) are coupled.

Second, the boundary conditions $(5.4 \mathrm{~h})$ on $\Gamma_{\mathrm{C}}$ for the discrete enthalpy equation involve $z_{\tau}^{k}$. In this way, (5.4f) is coupled with (5.4i), hence the whole system is coupled. Nonetheless, the mechanical part of system (5.4) (viz., $(5.4 \mathrm{a}-\mathrm{e}, \mathrm{i})$ ) can be reformulated in terms of the subdifferential inclusion

$$
\partial T_{\text {kin }}\left(\mathrm{D}_{t}^{2} u_{\tau}^{k}\right)+\partial_{(\dot{u}, \dot{z})} \Xi\left(u_{\tau}^{k} ; e\left(\mathrm{D}_{t} u_{\tau}^{k}\right), \mathrm{D}_{t} z_{\tau}^{k}\right)+\partial_{(u, z)} \Psi\left(u_{\tau}^{k}, z_{\tau}^{k-1}, \vartheta_{\tau}^{k}\right) \ni L_{\tau}^{k}
$$

(cf. (2.16)), i.e. it is semi-implicit w.r.t. the variable $z$. This will allow for some simplifications in the a priori estimates, see Lemma 5.5.

Third, we have added the term $\tau \operatorname{div}^{2}\left(\left|\nabla e\left(u_{\tau}^{k}\right)\right|^{\gamma-2} \mathbb{I} \nabla e\left(u_{\tau}^{k}\right)\right)-\tau \operatorname{div}\left(\left|e\left(u_{\tau}^{k}\right)\right|^{\gamma-2} e\left(u_{\tau}^{k}\right)\right)$ to the momentum equation in the bulk and to the corresponding boundary/contact conditions, too. Its role is to compensate the quadratic growth of the right-hand side of the enthalpy equation (5.4f) when $\gamma$ is chosen large enough, cf. the proof of Lemma 5.4. Being premultiplied by the factor $\tau$, this higher-homogeneity regularization will vanish when passing $\tau \rightarrow 0$. Because of this term, we also need to regularize the initial condition $u_{0}$ in (5.3), cf. (5.2).

REMARK 5.3 The time-discrete scheme (5.4) is simpler than the one devised in [35], where the first term on the right-hand side of $(5.4 \mathrm{f})$ was multiplied by the coefficient $(1-\sqrt{\tau})$, and additional terms (featuring monotone functions of $z$ and $\llbracket u \rrbracket$ ) were added to the discrete flow rule and to the boundary conditions on $\Gamma_{\mathrm{C}}$ for $u$. Such terms were used in the derivation of the discrete a priori estimates (in 
particular, of the discrete energy inequalities) via auxiliary minimization problems. Instead, here we adopt a more direct approach in the proof of the discrete mechanical and total energy inequalities, cf. (5.16) and (5.13) below. Indeed, we strongly rely on the semi-implicit character of (5.6) and on the convexity of $\alpha_{0}$.

Lemma 5.4 (Existence of weak solutions to Problem 5.1) Under the assumptions of Theorem 4.2, for every $k=1, \ldots, K_{\tau}$, there exists a triple $\left(u_{\tau}^{k}, z_{\tau}^{k}, \vartheta_{\tau}^{k}\right) \in W_{\Gamma_{\mathrm{D}}}^{2, \gamma}\left(\Omega \backslash \Gamma_{\mathrm{C}} ; \mathbb{R}^{3}\right) \times L^{\infty}\left(\Gamma_{\mathrm{C}}\right) \times$ $W^{1,2}\left(\Omega \backslash \Gamma_{\mathrm{C}}\right)$, fulfilling the weak formulation of the boundary value problem (5.4a)-(5.4i). Moreover, $\vartheta_{\tau}^{k} \geqslant 0$ a.e. in $\Omega$. If, in addition, (4.7) holds, then there exists some constant $\chi^{*}>0$ such that, for sufficiently small $\tau$,

$$
\vartheta_{\tau}^{k} \geqslant \chi^{*}>0 \quad \text { a.e. in } \Omega \text { for every } k=1, \ldots, K_{\tau} .
$$

Sketch of the proof. We may argue along the very same lines as in the proof of [35, Lemma 7.4]. Indeed, the existence of a weak solution to Problem 5.1 follows from the theory of pseudomonotone set-valued operators (see e.g. [36, Chap. 2]), and in particular from LerayLions type theorems, like [36, Chap. 5, Cor. 5.17]. To apply such results, one has to verify the strict monotonicity of the main part of the elliptic operator, involved in the weak formulation of problem (5.4a)-(5.4i). One has also to show that this operator is coercive w.r.t. the norm of $W_{\Gamma_{\mathrm{D}}}^{2, \gamma}\left(\Omega \backslash \Gamma_{\mathrm{C}} ; \mathbb{R}^{3}\right) \times L^{\infty}\left(\Gamma_{\mathrm{C}}\right) \times W^{1,2}\left(\Omega \backslash \Gamma_{\mathrm{C}}\right)$. For this, the term $\tau \operatorname{div}^{2}\left(\left|\nabla e\left(u_{\tau}^{k}\right)\right|^{\gamma-2} \mathbb{I} \nabla e\left(u_{\tau}^{k}\right)\right)-$ $\tau \operatorname{div}\left(\left|e\left(u_{\tau}^{k}\right)\right|^{\gamma-2} e\left(u_{\tau}^{k}\right)\right)$ on the left-hand side of (5.4a) plays a crucial role, in that it counteracts the quadratic nonlinearities in $e\left(u_{\tau}^{k}\right)$ and in $\nabla e\left(u_{\tau}^{k}\right)$ on the right-hand side of (5.4f): for this, we need $\gamma>\max \left\{4, \frac{2 \omega}{\omega-1}\right\}$. All the calculations for proving this strict monotonicity and coercivity in the present setting are very similar to those carried out in the proof of [35, Lemma 7.4]. The argument for the strict positivity (5.7), for which the growth property (4.2) is crucial, is also taken from [35, Lemma 7.4], to which we refer for all details (the reader should not be confused by an an unfortunate typo in [35, Formula (5.2)], though).

Approximate solutions. For $\tau>0$ fixed, the left-continuous and right-continuous piecewise constant, and the piecewise linear interpolants of the discrete solutions $\left\{u_{\tau}^{k}\right\}_{k=1}^{K_{\tau}}$ are respectively the functions $\bar{u}_{\tau}:(0, T) \rightarrow W_{\Gamma_{\mathrm{D}}}^{2, \gamma}\left(\Omega \backslash \Gamma_{\mathrm{C}} ; \mathbb{R}^{3}\right), \underline{u}_{\tau}:(0, T) \rightarrow W_{\Gamma_{\mathrm{D}}}^{2, \gamma}\left(\Omega \backslash \Gamma_{\mathrm{C}} ; \mathbb{R}^{3}\right)$, and $u_{\tau}:(0, T) \rightarrow$ $W_{\Gamma_{\mathrm{D}}}^{2, \gamma}\left(\Omega \backslash \Gamma_{\mathrm{c}} ; \mathbb{R}^{3}\right)$ defined by $\bar{u}_{\tau}(t):=u_{\tau}^{k}, \underline{u}_{\tau}(t):=u_{\tau}^{k-1}, u_{\tau}(t):=\frac{t-t_{\tau}^{k-1}}{\tau} u_{\tau}^{k}+\frac{t_{\tau}^{k}-t}{\tau} u_{\tau}^{k-1}$ for $t \in$ $\left(t_{\tau}^{k-1}, t_{\tau}^{k}\right]$. In the same way, we shall denote by $\bar{\vartheta}_{\tau}, \underline{\vartheta}_{\tau}$, and $\bar{z}_{\tau}$, the piecewise constant interpolants of the elements $\left\{\vartheta_{\tau}^{k}\right\}_{k=1}^{K_{\tau}}$ and $\left\{z_{\tau}^{k}\right\}_{k=1}^{K_{\tau}}$, and the related piecewise linear interpolants by $\vartheta_{\tau}$ and $z_{\tau}$. Furthermore, we shall use the notation $\bar{t}_{\tau}$ and $\underline{t}_{\tau}$ for the left-continuous and right-continuous piecewise constant interpolants associated with the partition, i.e. $\bar{t}_{\tau}(t)=t_{\tau}^{k}$ if $t_{\tau}^{k-1}<t \leqslant t_{\tau}^{k}$ and $\underline{t}_{\tau}(t)=t_{\tau}^{k-1}$ if $t_{\tau}^{k-1} \leqslant t<t_{\tau}^{k}$.

We shall also consider the interpolants $\bar{F}_{\tau}, \bar{f}_{\tau}$, and $f_{\tau}$, of the $K_{\tau}$-tuples $\left\{F_{\tau}^{k}\right\}_{k=1}^{K_{\tau}},\left\{f_{\tau}^{k}\right\}_{k=1}^{K_{\tau}}$. In view of (3.6a)-(3.6b) and (4.4a), the following estimates and strong convergences hold as $\tau \rightarrow 0$ :

$$
\bar{F}_{\tau} \rightarrow F \begin{cases}\text { in } L^{1}\left(0, T ; L^{2}\left(\Omega ; \mathbb{R}^{3}\right)\right) & \text { if } \varrho=0 \\ \text { in } L^{p}\left(0, T ; L^{6 / 5}\left(\Omega ; \mathbb{R}^{3}\right)\right) \text { for all } 1 \leqslant p<\infty & \text { if } \varrho>0\end{cases}
$$




$$
\begin{aligned}
& \exists C>0 \forall \tau>0:\left\|\bar{f}_{\tau}\right\|_{L^{\infty}\left(0, T ; L^{4 / 3}\left(\Gamma_{\mathrm{N}} ; \mathbb{R}^{3}\right)\right)} \leqslant C\|f\|_{L^{\infty}\left(0, T ; L^{4 / 3}\left(\Gamma_{\mathrm{N}} ; \mathbb{R}^{3}\right)\right)}, \\
& \bar{f}_{\tau} \rightarrow f \quad \text { in } L^{p}\left(0, T ; L^{4 / 3}\left(\Gamma_{\mathrm{N}} ; \mathbb{R}^{3}\right)\right) \text { for all } 1 \leqslant p<\infty \text { as } \tau \rightarrow 0, \\
& \exists C>0 \forall \tau>0:\left\|\dot{f}_{\tau}\right\|_{L^{1}\left(0, T ; L^{4 / 3}\left(\Gamma_{\mathrm{N}} ; \mathbb{R}^{3}\right)\right)} \leqslant 2\|\dot{f}\|_{L^{1}\left(0, T ; L^{4 / 3}\left(\Gamma_{\mathrm{N}} ; \mathbb{R}^{3}\right)\right)}, \\
& \exists C>0 \forall \tau>0:\left\|\dot{F}_{\tau}\right\|_{L^{1}\left(0, T ; L^{6 / 5}\left(\Omega ; \mathbb{R}^{3}\right)\right)} \leqslant 2\|\dot{F}\|_{L^{1}\left(0, T ; L^{6 / 5}\left(\Omega ; \mathbb{R}^{3}\right)\right)} \quad \text { if } \varrho>0 .
\end{aligned}
$$

Finally, we shall construct the discrete data $\left\{g_{\tau}^{k}\right\}_{k=1}^{K_{\tau}} \subset H^{1 / 2}(\partial \Omega)^{*}$ in such a way that the related piecewise constant interpolants $\bar{g}_{\tau}$ fulfill

$$
\bar{g}_{\tau} \rightarrow g \quad \text { in } L^{1}(\Sigma) \text { as } \tau \rightarrow 0 .
$$

Using the interpolants so far introduced, we now state the discrete versions of the weak formulation (3.11b) of the momentum inclusion, the total energy balance (3.11c), the semistability (3.11d), the weak formulation (3.11e) of the enthalpy equation. For the momentum inclusion, we introduce "discrete test functions", viz. $K_{\tau}$-tuples

$$
\left\{v_{\tau}^{k}\right\}_{k=1}^{K_{\tau}} \subset W_{\Gamma_{\mathrm{D}}}^{2,2}\left(\Omega \backslash \Gamma_{\mathrm{C}} ; \mathbb{R}^{3}\right) \text { fulfilling } \llbracket v_{\tau}^{k} \rrbracket \succeq 0 \text { on } \Gamma_{\mathrm{C}},
$$

and we denote by $\bar{v}_{\tau}$ and $v_{\tau}$ their interpolants. Furthermore, referring to the definition (3.8) of $\Phi$, we shall use the notation

$$
\Phi_{\tau}(u, z):=\Phi(u, z)+\frac{\tau}{\gamma} \int_{\Omega \backslash \Gamma_{\mathrm{C}}}|e(u)|^{\gamma}+|\nabla e(u)|^{\gamma} \mathrm{d} x .
$$

Hence, the approximate solutions $\left(\bar{u}_{\tau}, \underline{u}_{\tau}, \bar{\vartheta}_{\tau}, \underline{\vartheta}_{\tau}, \bar{z}_{\tau}, u_{\tau}, \vartheta_{\tau}, z_{\tau}\right.$ ) fulfill the discrete (weak) momentum inclusion

$$
\begin{aligned}
\int_{Q}( & \left(\mathbb{D} e\left(\dot{u}_{\tau}\right)+\mathbb{C} e\left(\bar{u}_{\tau}\right)-\mathbb{B} \Theta\left(\bar{\vartheta}_{\tau}\right)+\tau\left|e\left(\bar{u}_{\tau}\right)\right|^{\gamma-2} e\left(\bar{u}_{\tau}\right)\right): e\left(\bar{v}_{\tau}-\bar{u}_{\tau}\right) \\
& \left.+\left(\left(\mathbb{H}+\tau\left|\nabla e\left(\bar{u}_{\tau}\right)\right|^{\gamma-2} \mathbb{I}\right) \nabla e\left(\bar{u}_{\tau}\right)+\mathbb{G} \nabla e\left(\dot{u}_{\tau}\right)\right): \nabla e\left(\bar{v}_{\tau}-\bar{u}_{\tau}\right)\right) \mathrm{d} x \mathrm{~d} t \\
& +\int_{\Sigma_{\mathrm{C}}} \underline{z}_{\tau} \alpha_{0}^{\prime}\left(\left[\bar{u}_{\tau}\right]\right) \cdot\left[\bar{v}_{\tau}-\bar{u}_{\tau}\right] \mathrm{d} x \mathrm{~d} t-\int_{\tau}^{T} \int_{\Omega} \varrho \dot{u}_{\tau}(\cdot-\tau) \cdot\left(\dot{v}_{\tau}-\dot{u}_{\tau}\right) \mathrm{d} x \mathrm{~d} t \\
& +\int_{\Omega} \varrho \dot{u}_{\tau}(T) \cdot\left(v_{\tau}(T)-u_{\tau}(T)\right) \mathrm{d} x \\
\geqslant & \int_{\Omega} \varrho \dot{u}_{0} \cdot\left(v_{\tau}(\tau)-u_{\tau}(\tau)\right) \mathrm{d} x+\int_{Q} \bar{F}_{\tau} \cdot\left(\bar{v}_{\tau}-\bar{u}_{\tau}\right) \mathrm{d} x \mathrm{~d} t+\int_{\Sigma_{\mathrm{N}}} \bar{f}_{\tau} \cdot\left(\bar{v}_{\tau}-\bar{u}_{\tau}\right) \mathrm{d} S \mathrm{~d} t
\end{aligned}
$$

for all $K_{\tau}$-tuples $\left\{v_{\tau}^{k}\right\}_{k=1}^{K_{\tau}} \subset W_{\Gamma_{\mathrm{D}}}^{2,2}\left(\Omega \backslash \Gamma_{\mathrm{c}} ; \mathbb{R}^{3}\right)$ fulfilling (5.10);

the discrete total energy inequality

$$
\begin{aligned}
T_{\text {kin }}\left(\dot{u}_{\tau}(t)\right)+\Phi_{\tau}\left(\bar{u}_{\tau}(t), \bar{z}_{\tau}(t)\right) & +\int_{\Omega} \bar{\vartheta}_{\tau}(t) \mathrm{d} x \leqslant T_{\text {kin }}\left(\dot{u}_{0}\right)+\Phi_{\tau}\left(u_{0, \tau}, z_{0}\right) \\
& +\int_{\Omega} \vartheta_{0} \mathrm{~d} x+\int_{0}^{\bar{t}_{\tau}(t)}\left(\int_{\Omega} \bar{F}_{\tau} \cdot \dot{u}_{\tau} \mathrm{d} x+\int_{\Gamma_{\mathrm{N}}} \bar{f}_{\tau} \cdot \dot{u}_{\tau} \mathrm{d} S+\int_{\partial \Omega} \bar{g}_{\tau} \mathrm{d} S\right) \mathrm{d} s
\end{aligned}
$$


(see (2.15) for the definition of $\left.T_{\text {kin }}\right)$, the discrete semistability for a.a. $t \in(0, T)$

$$
\Phi_{\tau}\left(\bar{u}_{\tau}(t), \bar{z}_{\tau}(t)\right) \leqslant \Phi_{\tau}\left(\bar{u}_{\tau}(t), \tilde{z}\right)+\mathbb{R}\left(\llbracket \bar{u}_{\tau}(t) \rrbracket, \tilde{z}-\bar{z}_{\tau}(t)\right) \quad \text { for all } \tilde{z} \in L^{\infty}\left(\Gamma_{\mathrm{C}}\right)
$$

the discrete (weak) enthalpy equation

$$
\begin{aligned}
\int_{\Omega} \vartheta_{\tau}(T) w(T) \mathrm{d} x & \left.\left.+\int_{Q} \mathcal{K}\left(e\left(\bar{u}_{\tau}\right), \bar{\vartheta}_{\tau}\right) \nabla \bar{\vartheta}_{\tau} \cdot \nabla w-\vartheta_{\tau} \dot{w} \mathrm{~d} x \mathrm{~d} t+\int_{\Sigma_{\mathrm{C}}} \eta\left(\llbracket \underline{u}_{\tau}\right], \bar{z}_{\tau}\right) \llbracket \Theta\left(\bar{\vartheta}_{\tau}\right)\right][w] \mathrm{d} S \mathrm{~d} t \\
= & \int_{Q}\left(\mathbb{D} e\left(\dot{u}_{\tau}\right): e\left(\dot{u}_{\tau}\right)-\Theta\left(\bar{\vartheta}_{\tau}\right) \mathbb{B}: e\left(\dot{u}_{\tau}\right)+\mathbb{G} \nabla e\left(\dot{u}_{\tau}\right) \vdots \nabla e\left(\dot{u}_{\tau}\right)\right) w \mathrm{~d} x \mathrm{~d} t \\
& -\int_{\Sigma_{\mathrm{C}}} a_{1}\left(\left[\bar{u}_{\tau}\right]\right) \dot{z}_{\tau} \frac{\left.w\right|_{\Gamma_{\mathrm{C}}} ^{+}+\left.w\right|_{\Gamma_{\mathrm{C}}} ^{-}}{2} \mathrm{~d} S \mathrm{~d} t+\int_{\Omega} \vartheta_{0} w(0) \mathrm{d} x+\int_{\Sigma} \bar{g}_{\tau} w \mathrm{~d} S \mathrm{~d} t
\end{aligned}
$$

with $w$ qualified as in (3.11e). Inequality (5.12) can be obtained from (5.4a-e), by using a suitable discrete "by-part" summation formula, cf. [39, Formula (4.49)]. We now prove (5.13) and (5.14).

LEMmA 5.5 (Approximate energetics) Let $\varrho \geqslant 0$. Under the assumptions of Theorem 4.2, for all $\tau>0$ the approximate solutions $\left(\bar{u}_{\tau}, \underline{u}_{\tau}, \bar{\vartheta}_{\tau}, \bar{z}_{\tau}, u_{\tau}, \vartheta_{\tau}, z_{\tau}\right)$ fulfill the following discrete mechanical energy inequality

$$
\begin{aligned}
& T_{\text {kin }}\left(\dot{u}_{\tau}(t)\right)+\Phi_{\tau}\left(\bar{u}_{\tau}(t), \bar{z}_{\tau}(t)\right) \\
& \left.\quad+\int_{0}^{\bar{t}_{\tau}(t)}\left(\int_{\Omega} \mathbb{D} e\left(\dot{u}_{\tau}\right): e\left(\dot{u}_{\tau}\right)+\mathbb{G} \nabla e\left(\dot{u}_{\tau}\right) \vdots \nabla e\left(\dot{u}_{\tau}\right) \mathrm{d} x+\int_{\Gamma_{\mathrm{C}}} \zeta_{1}\left(\llbracket \bar{u}_{\tau}\right], \dot{z}_{\tau}\right) \mathrm{d} S\right) \mathrm{d} s \\
& \leqslant T_{\text {kin }}\left(\dot{u}_{0}\right)+\Phi_{\tau}\left(u_{0, \tau}, z_{0}\right) \\
& \quad+\int_{0}^{\bar{t}_{\tau}(t)}\left(\int_{\Omega} \Theta\left(\bar{\vartheta}_{\tau}\right) \mathbb{B}: e\left(\dot{u}_{\tau}\right) \mathrm{d} x+\int_{\Omega} \bar{F}_{\tau} \cdot \dot{u}_{\tau} \mathrm{d} x+\int_{\Gamma_{\mathrm{N}}} \bar{f}_{\tau} \cdot \dot{u}_{\tau} \mathrm{d} S\right) \mathrm{d} s,
\end{aligned}
$$

(see (2.12b) for the definition of $\zeta_{1}$ ), the discrete total energy inequality (5.13), and the discrete semistability (5.14).

Proof. Preliminarily, we observe that (5.4i) is the Euler-Lagrange equation for the minimum problem

$$
\left.\left.z_{\tau}^{k} \in \operatorname{Argmin}_{z \in L^{\infty}\left(\Gamma_{\mathrm{C}}\right)} \int_{\Gamma_{\mathrm{C}}} z \alpha_{0}\left(\llbracket u_{\tau}^{k}\right]\right)-z a_{1}\left(\llbracket u_{\tau}^{k}\right]\right)+\mathcal{F}\left(z_{\tau}^{k-1} ; z\right) \mathrm{d} S,
$$

where $\mathcal{F}\left(z_{\tau}^{k-1} ; z\right)$ is as in (5.5). Therefore, we have

$$
\begin{aligned}
& \int_{\Gamma_{\mathrm{C}}} I_{(\infty, 0]}\left(\frac{z_{\tau}^{k}-z_{\tau}^{k-1}}{\tau}\right)+z_{\tau}^{k} \alpha_{0}\left(\llbracket u_{\tau}^{k} \rrbracket\right)-z_{\tau}^{k} a_{1}\left(\llbracket u_{\tau}^{k} \rrbracket\right)+I_{[0,1]}\left(z_{\tau}^{k}\right) \mathrm{d} S \\
& \leqslant \int_{\Gamma_{\mathrm{C}}} z_{\tau}^{k-1} \alpha_{0}\left(\llbracket u_{\tau}^{k} \rrbracket\right)-z_{\tau}^{k-1} a_{1}\left(\llbracket u_{\tau}^{k} \rrbracket\right) \mathrm{d} S .
\end{aligned}
$$

To prove (5.16), we test the boundary-value problem (5.4a)-(5.4e) by $u_{\tau}^{k}-u_{\tau}^{k-1}$. We add to the 
resulting inequality the previously observed (5.18), thus obtaining

$$
\begin{aligned}
\varrho \int_{\Omega} & \mathrm{D}_{t}^{2} u_{\tau}^{k} \cdot \mathrm{D}_{t} u_{\tau}^{k} \mathrm{~d} x+\int_{\Omega} \mathbb{D} e\left(\mathrm{D}_{t} u_{\tau}^{k}\right): e\left(\mathrm{D}_{t} u_{\tau}^{k}\right)+\mathbb{G} \nabla e\left(\mathrm{D}_{t} u_{\tau}^{k}\right): \nabla e\left(\mathrm{D}_{t} u_{\tau}^{k}\right) \mathrm{d} x \\
& +\int_{\Omega} \mathbb{C} e\left(u_{\tau}^{k}\right): e\left(\mathrm{D}_{t} u_{\tau}^{k}\right)+\mathbb{H} \nabla e\left(u_{\tau}^{k}\right): \nabla e\left(\mathrm{D}_{t} u_{\tau}^{k}\right) \mathrm{d} x \\
& +\tau \int_{\Omega}\left|e\left(u_{\tau}^{k}\right)\right|^{\gamma-2} e\left(u_{\tau}^{k}\right): e\left(\mathrm{D}_{t} u_{\tau}^{k}\right)+\left|\nabla e\left(u_{\tau}^{k}\right)\right|^{\gamma-2} \nabla e\left(u_{\tau}^{k}\right): \nabla e\left(\mathrm{D}_{t} u_{\tau}^{k}\right) \mathrm{d} x \\
& +\int_{\Gamma_{\mathrm{C}}} z_{\tau}^{k-1} \alpha_{0}^{\prime}\left(\llbracket u_{\tau}^{k} \rrbracket\right) \cdot \llbracket \mathrm{D}_{t} u_{\tau}^{k} \rrbracket+\left(\mathrm{D}_{t} z_{\tau}^{k}\right) \alpha_{0}\left(\llbracket u_{\tau}^{k} \rrbracket\right) \mathrm{d} S-\int_{\Gamma_{\mathrm{C}}} a_{1}\left(\llbracket u_{\tau}^{k} \rrbracket\right)\left(\mathrm{D}_{t} z_{\tau}^{k}\right) \mathrm{d} S \\
= & : I_{1}+I_{2}+I_{3}+I_{4}+I_{5}+I_{6} \\
\leqslant & \tau \int_{\Omega} \mathbb{B} \Theta\left(\vartheta_{\tau}^{k}\right): e\left(\mathrm{D}_{t} u_{\tau}^{k}\right) \mathrm{d} x+\tau \int_{\Omega} F_{\tau}^{k} \cdot \mathrm{D}_{t} u_{\tau}^{k} \mathrm{~d} x+\tau \int_{\Gamma_{\mathrm{N}}} f_{\tau}^{k} \cdot \mathrm{D}_{t} u_{\tau}^{k} \mathrm{~d} S .
\end{aligned}
$$

Now, we estimate the terms $I_{i}, i=1, \ldots, 6$. First of all, we observe that

$$
I_{1} \geqslant \frac{\varrho}{2 \tau} \int_{\Omega}\left|\mathrm{D}_{t} u_{\tau}^{k}\right|^{2} \mathrm{~d} x-\frac{\varrho}{2 \tau} \int_{\Omega}\left|\mathrm{D}_{t} u_{\tau}^{k-1}\right|^{2} \mathrm{~d} x=\frac{\varrho}{2} \mathrm{D}_{t} \int_{\Omega}\left|\mathrm{D}_{t} u_{\tau}^{k}\right|^{2} \mathrm{~d} x
$$

Clearly, upon summation $I_{2}$ will yield the third summand on the right-hand side of (5.16), whereas we observe that

$$
\left.\begin{array}{l}
I_{3} \geqslant \mathrm{D}_{t} \int_{\Omega} \frac{1}{2}\left(\mathbb{C} e\left(u_{\tau}^{k}\right): e\left(u_{\tau}^{k}\right)+\mathbb{H} \nabla\left(e\left(u_{\tau}^{k}\right)\right): \nabla\left(e\left(u_{\tau}^{k}\right)\right)\right) \mathrm{d} x, \\
I_{4} \geqslant \mathrm{D}_{t} \int_{\Omega} \frac{\tau}{\gamma}\left|e\left(u_{\tau}^{k}\right)\right|^{\gamma}+\frac{\tau}{\gamma}\left|\nabla e\left(u_{\tau}^{k}\right)\right|^{\gamma} \mathrm{d} x,
\end{array}\right\}
$$

where we have used elementary convex-analysis inequalities. Now, it follows from the convexity of $\alpha_{0}$, cf. (4.1g), that

$$
\left.\left.\int_{\Gamma_{\mathrm{C}}} z_{\tau}^{k-1} \alpha_{0}^{\prime}\left(\llbracket u_{\tau}^{k}\right]\right) \cdot\left[\mathrm{D}_{t} u_{\tau}^{k}\right] \mathrm{d} S \geqslant \frac{1}{\tau} \int_{\Gamma_{\mathrm{C}}} z_{\tau}^{k-1} \alpha_{0}\left(\left[u_{\tau}^{k}\right]\right) \mathrm{d} S-\frac{1}{\tau} \int_{\Gamma_{\mathrm{C}}} z_{\tau}^{k-1} \alpha_{0}\left(\llbracket u_{\tau}^{k-1}\right]\right) \mathrm{d} S .
$$

Hence, taking into account the cancellation of the term $\int_{\Gamma_{\mathrm{C}}} z_{\tau}^{k-1} \alpha_{0}\left(\llbracket u_{\tau}^{k} \rrbracket\right)$, for $I_{5}$ we conclude the following inequality

$$
I_{5} \geqslant \mathrm{D}_{t} \int_{\Gamma_{\mathrm{C}}} z_{\tau}^{k} \alpha_{0}\left(\llbracket u_{\tau}^{k} \rrbracket\right) \mathrm{d} S
$$


Combining (5.19)-(5.22), rearranging terms and multiplying by $\tau$, we obtain

$$
\begin{aligned}
\int_{\Omega} \frac{\varrho}{2}\left|\mathrm{D}_{t} u_{\tau}^{k}\right|^{2}+\tau \mathbb{D} e\left(\mathrm{D}_{t} u_{\tau}^{k}\right): e\left(\mathrm{D}_{t} u_{\tau}^{k}\right)+\tau \mathbb{G} \nabla e\left(\mathrm{D}_{t} u_{\tau}^{k}\right): \nabla e\left(\mathrm{D}_{t} u_{\tau}^{k}\right)+\frac{1}{2} \mathbb{C} e\left(u_{\tau}^{k}\right): e\left(u_{\tau}^{k}\right)+\frac{\tau}{\gamma}\left|e\left(u_{\tau}^{k}\right)\right|^{\gamma} \\
\left.\left.+\frac{\tau}{\gamma}\left|\nabla e\left(u_{\tau}^{k}\right)\right|^{\gamma}+\frac{1}{2} \mathbb{H} \nabla\left(e\left(u_{\tau}^{k}\right)\right): \nabla\left(e\left(u_{\tau}^{k}\right)\right) \mathrm{d} x+\int_{\Gamma_{\mathrm{C}}} z_{\tau}^{k} \alpha_{0}\left(\llbracket u_{\tau}^{k}\right]\right) \mathrm{d} S-\int_{\Gamma_{\mathrm{C}}} \tau a_{1}\left(\llbracket u_{\tau}^{k}\right]\right)\left(\mathrm{D}_{t} z_{\tau}^{k}\right) \mathrm{d} S \\
\leqslant \int_{\Omega} \frac{\varrho}{2}\left|\mathrm{D}_{t} u_{\tau}^{k-1}\right|^{2}+\frac{1}{2} \int_{\Omega} \mathbb{C} e\left(u_{\tau}^{k-1}\right): e\left(u_{\tau}^{k-1}\right)+\frac{\tau}{\gamma}\left|e\left(u_{\tau}^{k-1}\right)\right|^{\gamma}+\frac{\tau}{\gamma}\left|\nabla e\left(u_{\tau}^{k-1}\right)\right|^{\gamma} \\
+\frac{1}{2} \mathbb{H} \nabla\left(e\left(u_{\tau}^{k-1}\right)\right) \vdots \nabla\left(e\left(u_{\tau}^{k-1}\right)\right) \mathrm{d} x+\int_{\Gamma_{\mathrm{C}}} z_{\tau}^{k-1} \alpha_{0}\left(\left[u_{\tau}^{k-1} \rrbracket\right) \mathrm{d} S\right. \\
+\tau \int_{\Omega} \mathbb{B} \Theta\left(\vartheta_{\tau}^{k}\right): e\left(\mathrm{D}_{t} u_{\tau}^{k}\right) \mathrm{d} x+\tau \int_{\Omega} F_{\tau}^{k} \cdot \mathrm{D}_{t} u_{\tau}^{k} \mathrm{~d} x+\tau \int_{\Gamma_{\mathrm{N}}} f_{\tau}^{k} \cdot \mathrm{D}_{t} u_{\tau}^{k} \mathrm{~d} S
\end{aligned}
$$

Summing over the index $k$, we conclude (5.16).

In order to obtain (5.13) for a fixed $t \in(0, T)$, we test (5.15), integrated on the time-interval $\left(0, \bar{t}_{\tau}(t)\right)$, by 1 , and add the resulting relation to the mechanical energy equality (5.16). Taking into account all cancellations, we immediately conclude (5.13).

Eventually, from (5.18) and the degree-1 homogeneity of $\mathbb{R}\left(\llbracket u_{\tau}^{k} \rrbracket, \cdot\right)$, it also follows that

$$
\begin{aligned}
\Phi_{\tau}\left(u_{\tau}^{k}, z_{\tau}^{k}\right) & \left.\left.\leqslant \Phi_{\tau}\left(u_{\tau}^{k}, \tilde{z}\right)-\int_{\Gamma_{\mathrm{C}}} a_{1}\left(\llbracket u_{\tau}^{k}\right]\right)\left(\tilde{z}-z_{\tau}^{k-1}\right) \mathrm{d} S-\int_{\Gamma_{\mathrm{C}}} a_{1}\left(\llbracket u_{\tau}^{k}\right]\right)\left(z_{\tau}^{k-1}-z_{\tau}^{k}\right) \mathrm{d} S \\
& \left.\left.=\Phi_{\tau}\left(u_{\tau}^{k}, \tilde{z}\right)-\int_{\Gamma_{\mathrm{C}}} a_{1}\left(\llbracket u_{\tau}^{k}\right]\right)\left(\tilde{z}-z_{\tau}^{k}\right) \mathrm{d} S=\Phi_{\tau}\left(u_{\tau}^{k}, \tilde{z}\right)+\mathbb{R}\left(\llbracket u_{\tau}^{k}\right], \tilde{z}-z_{\tau}^{k}\right)
\end{aligned}
$$

for all $\tilde{z} \leqslant z_{\tau}^{k}$ a.e. on $\Gamma_{\mathrm{C}}$. This is the discrete version of (5.14).

We conclude this section with a result collecting all the a priori estimates on the approximate solutions.

Lemma 5.6 (A priori estimates) Under the assumptions of Theorem 4.2, for all $\varrho \geqslant 0$ and $\tau>0$, the approximate solutions $\left(\bar{u}_{\tau}, \bar{\vartheta}_{\tau}, \bar{z}_{\tau}, u_{\tau}, \vartheta_{\tau}, z_{\tau}\right)$ satisfy

$$
\begin{aligned}
& \left\|\bar{u}_{\tau}\right\|_{L^{\infty}\left(0, T ; W_{\Gamma_{\mathrm{D}}}^{2,2}\left(\Omega ; \mathbb{R}^{3}\right)\right)} \leqslant S_{0}, \\
& \left\|u_{\tau}\right\|_{W^{1,2}\left(0, T ; W_{\Gamma_{\mathrm{D}}}^{2,2}\left(\Omega ; \mathbb{R}^{3}\right)\right)} \leqslant S_{0}, \\
& \varrho^{1 / 2}\left\|u_{\tau}\right\|_{W^{1, \infty}\left(0, T ; L^{2}\left(\Omega ; \mathbb{R}^{3}\right)\right)} \leqslant S_{0}, \\
& \left\|\bar{u}_{\tau}\right\|_{L^{\infty}\left(0, T ; W_{\Gamma_{\mathrm{D}}}^{2, \gamma}\left(\Omega ; \mathbb{R}^{3}\right)\right)} \leqslant \frac{S_{0}}{\tau^{1 / \gamma},} \\
& \left\|\bar{z}_{\tau}\right\|_{L^{\infty}\left(\Sigma_{\mathrm{C}}\right)} \leqslant S_{0}, \\
& \left\|\bar{z}_{\tau}\right\|_{B V\left([0, T] ; L^{1}\left(\Gamma_{\mathrm{C}}\right)\right)} \leqslant S_{0}, \\
& \left\|\bar{\vartheta}_{\tau}\right\|_{L^{\infty}\left(0, T ; L^{1}(\Omega)\right)} \leqslant S_{0}, \\
& \left\|\bar{\vartheta}_{\tau}\right\|_{L^{r}\left(0, T ; W^{1, r}(\Omega)\right)} \leqslant S_{r} \text { for any } 1 \leqslant r<\frac{5}{4}, \\
& \left\|\dot{\vartheta}_{\tau}\right\|_{L^{1}\left(0, T ; W^{1, r^{\prime}}(\Omega)^{*}\right)} \leqslant S_{0},
\end{aligned}
$$




$$
\varrho\left\|\dot{u}_{\tau}\right\|_{B V\left([0, T] ; W_{\Gamma_{\mathrm{D}}}^{2, \nu}\left(\Omega ; \mathbb{R}^{3}\right)^{*}\right)} \leqslant S_{0},
$$

for some constants $S_{0}>0$ and $S_{r}>0$ independent of $\tau$. Estimates $\left(5.25 \mathrm{e}, \mathrm{f}\right.$ ) hold for $z_{\tau}$ as well, and so do estimates $(5.25 \mathrm{~g}, \mathrm{~h})$ for $\vartheta_{\tau}$.

Proof. We only sketch the calculations for proving (5.25), since the argument closely follows the proof of [35, Lemma 7.7], to which we shall systematically refer.

First of all, we use the "discrete total energy" balance (5.13). Clearly, the first summand on the left-hand side provides a bound for the quantity $\varrho^{1 / 2}\left\|u_{\tau}\right\|_{W^{1, \infty}\left(0, T ; L^{2}\left(\Omega ; \mathbb{R}^{3}\right)\right)}$. Secondly, we observe that (cf. (3.8), (5.11)),

$$
\begin{aligned}
& \Phi_{\tau}(u, z) \geqslant C\left(\int_{\Omega}|e(u)|^{2}+|\nabla e(u)|^{2}+\tau|e(u)|^{\gamma}+\tau|\nabla e(u)|^{\gamma} \mathrm{d} x\right. \\
& \left.+\int_{\Gamma_{\mathrm{C}}} z|\llbracket u \rrbracket|^{2} \mathrm{~d} S\right)-C_{a_{0}} \int_{\Gamma_{\mathrm{C}}} z|\llbracket u \rrbracket| \mathrm{d} S-C_{a_{0}}^{\prime} \\
& \geqslant C\left(\|u\|_{W^{1,2}\left(\Omega ; \mathbb{R}^{3}\right)}^{2}+\|u\|_{W^{2,2}\left(\Omega ; \mathbb{R}^{3}\right)}^{2}+\tau\|u\|_{W^{2, \nu}\left(\Omega ; \mathbb{R}^{3}\right)}^{\gamma}+\int_{\Gamma_{\mathrm{C}}} z|\llbracket u \rrbracket|^{2} \mathrm{~d} S\right)-C^{\prime},
\end{aligned}
$$

where we have used the positive-definiteness of $\mathbb{A}, \mathbb{C}$, and $\mathbb{H}$, and the growth condition (4.1f), to derive the first inequality. The second estimate ensues from Korn's inequality, and from absorbing the term $\int_{\Gamma_{\mathrm{C}}} z|\llbracket u \rrbracket| \mathrm{d} x$ into $\int_{\Gamma_{\mathrm{C}}} z|\llbracket u \rrbracket|^{2} \mathrm{~d} x$, since $z \in[0,1]$ a.e. on $\Gamma_{\mathrm{C}}$. Therefore, the second term on the left-hand side of (5.13) estimates $\left\|\bar{u}_{\tau}(t)\right\|_{W^{2,2}\left(\Omega ; \mathbb{R}^{3}\right)}^{2}$ and $\tau\left\|\bar{u}_{\tau}(t)\right\|_{W^{2, \nu}\left(\Omega ; \mathbb{R}^{3}\right)}^{\gamma}$ uniformly w.r.t. $t \in[0, T]$. Thirdly, $\bar{\vartheta}_{\tau} \geqslant 0$ a.e. in $\Omega$ thanks to Lemma 5.4 , hence the third term estimates $\left\|\bar{\vartheta}_{\tau}\right\|_{L^{\infty}\left(0, T ; L^{1}(\Omega)\right)}$. To deal with the right-hand side of (5.13), we use (3.7), (5.2) and, for the last integral term, (5.8) and (5.9), arguing in the very same way as in the proof of [35, Lemma 7.7]. We conclude applying the discrete Gronwall lemma, and thus obtain estimates (5.25a), (5.25c), and $(5.25 \mathrm{~g})$. Since $\bar{z}_{\tau} \in[0,1]$ a.e. on $\Sigma_{\mathrm{C}}$, we obviously have (5.25e).

Secondly, again arguing as for [39, Prop. 4.2] and [35, Lemma 7.7], we make use of the technique by Boccardo and Gallouët [3] with the simplification devised in [12], and we test the heat equation (5.15) by $\pi\left(\bar{\vartheta}_{\tau}\right)$, where $\pi:[0,+\infty) \rightarrow[0,1]$ is the map $w \mapsto \pi(w)=1-\frac{1}{(1+w)^{5}}$, for $\varsigma>0$. Since $\pi$ is Lipschitz continuous, $\pi\left(\bar{\vartheta}_{\tau}\right) \in W^{1,2}\left(\Omega \backslash \Gamma_{\mathrm{C}}\right)$ is an admissible test function. We thus have

$$
\begin{aligned}
\varsigma \mathrm{k} \int_{Q} \frac{\left|\nabla \bar{\vartheta}_{\tau}\right|^{2}}{\left(1+\bar{\vartheta}_{\tau}\right)^{1+\varsigma}} \mathrm{d} x \mathrm{~d} t \leqslant & \int_{Q} \mathcal{K}\left(e\left(\bar{u}_{\tau}\right), \bar{\vartheta}_{\tau}\right) \nabla \bar{\vartheta}_{\tau} \cdot \nabla \pi\left(\bar{\vartheta}_{\tau}\right) \mathrm{d} x \mathrm{~d} t \\
& +\int_{\Sigma_{\mathrm{C}}} \eta\left(\llbracket \underline{u}_{\tau} \rrbracket, \bar{z}_{\tau}\right)\left[\Theta\left(\bar{\vartheta}_{\tau}\right)\right]\left[\pi\left(\bar{\vartheta}_{\tau}\right) \rrbracket \mathrm{d} S \mathrm{~d} t+\int_{\Omega} \widehat{\pi}\left(\bar{\vartheta}_{\tau}(T, \cdot)\right) \mathrm{d} x\right. \\
\leqslant & \int_{\Omega} \widehat{\pi}\left(\vartheta_{0}\right) \mathrm{d} x+C\left(\left\|\mathbb{D} e\left(\dot{u}_{\tau}\right): e\left(\dot{u}_{\tau}\right)\right\|_{L^{1}(Q)}+\left\|\mathbb{G} \nabla e\left(\dot{u}_{\tau}\right) \vdots \nabla e\left(\dot{u}_{\tau}\right)\right\|_{L^{1}(Q)}\right. \\
& \left.\left.+\left\|\Theta\left(\bar{\vartheta}_{\tau}\right) \mathbb{B}: e\left(\dot{u}_{\tau}\right)\right\|_{L^{1}(Q)}+\| \zeta_{1}\left(\llbracket \bar{u}_{\tau}\right], \dot{z}_{\tau}\right) \|_{L^{1}\left(\Sigma_{\mathrm{C}}\right)}\right)+\left\|\bar{g}_{\tau}\right\|_{L^{1}(\Sigma)},
\end{aligned}
$$

where $\widehat{\pi}$ is the primitive function of $\pi$ such that $\widehat{\pi}(0)=0$. Note that inequality (5.27) follows from (4.1d), the fact that $\eta\left(\underline{u}_{\tau}, \bar{z}_{\tau}\right) \llbracket \Theta\left(\bar{\vartheta}_{\tau}\right) \rrbracket \llbracket \pi\left(\bar{\vartheta}_{\tau}\right) \rrbracket \geqslant 0$ a.e. in $\Sigma_{\mathrm{C}}$ (by the positivity of $\eta$ and the 
monotonicity of $\Theta$ and $\pi$ ), from the "discrete chain rule" [39, Formula (4.30)] for $\widehat{\pi}$, and from the fact $0 \leqslant \pi\left(\bar{\vartheta}_{\tau}\right) \leqslant 1$ a.e. in $\Omega$. Combining (5.27) and performing the very same calculations as in the proof of [39, Prop. 4.2], with the Gagliardo-Nirenberg inequality we find for all $1 \leqslant r<5 / 4$, that

$$
\begin{aligned}
\left\|\nabla \bar{\vartheta}_{\tau}\right\|_{L^{r}\left(Q ; \mathbb{R}^{3}\right)}^{r} \leqslant C_{r}\left(1+\left\|\mathbb{D} e\left(\dot{u}_{\tau}\right): e\left(\dot{u}_{\tau}\right)\right\|_{L^{1}(Q)}+\left\|\mathbb{G} \nabla e\left(\dot{u}_{\tau}\right): \nabla e\left(\dot{u}_{\tau}\right)\right\|_{L^{1}(Q)}\right. \\
\left.+\left\|\Theta\left(\bar{\vartheta}_{\tau}\right) \mathbb{B}: e\left(\dot{u}_{\tau}\right)\right\|_{L^{1}(Q)}+\left\|\zeta_{1}\left(\left[\bar{u}_{\tau}\right], \dot{z}_{\tau}\right)\right\|_{L^{1}\left(\Sigma_{C}\right)}\right)
\end{aligned}
$$

for some positive constant $C_{r}$, depending on $r$ and also on the function $\eta$, cf. (4.1e).

Then, we multiply (5.28) by a constant $\rho_{1}>0$ and add it to (5.16) (in which we set $t=T$ ). Now, by positive-definiteness of $\mathbb{D}$ and $\mathbb{G}$, the third term on the left-hand side of (5.16) is bounded from below by $c\left(\left\|e\left(\dot{u}_{\tau}\right)\right\|_{L^{2}\left(Q ; \mathbb{R}^{3 \times 3}\right)}^{2}+\left\|\nabla e\left(\dot{u}_{\tau}\right)\right\|_{L^{2}\left(Q ; \mathbb{R}^{3 \times 3 \times 3}\right)}^{2}\right)$, and it controls $\left\|\zeta_{1}\left(\llbracket \underline{u}_{\tau} \rrbracket, \dot{z}_{\tau}\right)\right\|_{L^{1}\left(\Sigma_{\mathrm{C}}\right)}$. Thus, we choose $\rho_{1}$ small enough in such a way as to absorb the second, the third, and the fifth term on the right-hand side of (5.28) into the left-hand side of (5.16). Hence, we find

$$
\begin{aligned}
& c\left(\left\|e\left(\dot{u}_{\tau}\right)\right\|_{L^{2}\left(Q ; \mathbb{R}^{3 \times 3}\right)}^{2}+\left\|\nabla e\left(\dot{u}_{\tau}\right)\right\|_{L^{2}\left(Q ; \mathbb{R}^{3 \times 3 \times 3}\right)}^{2}\right) \\
& +\left(1-\rho_{1}\right)\left\|\zeta_{1}\left(\left[\bar{u}_{\tau}\right], \dot{z}_{\tau}\right)\right\|_{L^{1}\left(\Sigma_{\mathrm{C}}\right)}+\rho_{1}\left\|\nabla \bar{\vartheta}_{\tau}\right\|_{L^{r}\left(Q ; \mathbb{R}^{3}\right)}^{r} \\
& \leqslant T_{\text {kin }}\left(\dot{u}_{0, \tau}\right)+\Phi_{\tau}\left(u_{0, \tau}, z_{0, \tau}\right)+\int_{Q} \bar{F}_{\tau} \cdot \dot{u}_{\tau} \mathrm{d} x \mathrm{~d} t \\
& +\int_{\Sigma_{\mathrm{N}}} \bar{f}_{\tau} \cdot \dot{u}_{\tau} \mathrm{d} S \mathrm{~d} t+\left(\rho_{1} C_{r}+1\right)\left\|\Theta\left(\bar{\vartheta}_{\tau}\right) \mathbb{B} e\left(\dot{u}_{\tau}\right)\right\|_{L^{1}(Q)} .
\end{aligned}
$$

The first two summands on the right-hand side of (5.29) are estimated in view of (3.7) and (5.2). Using (5.8), we handle the terms $\int_{Q} \bar{F}_{\tau} \cdot \dot{u}_{\tau} \mathrm{d} x \mathrm{~d} t$ and $\int_{\Sigma_{\mathrm{N}}} \bar{f}_{\tau} \cdot \dot{u}_{\tau} \mathrm{d} S \mathrm{~d} t$ in the very same way as in the proof of [35, Lemma 7.7]. Finally, we use

$$
\begin{aligned}
\left(\rho_{1} C_{r}+1\right)\left\|\Theta\left(\bar{\vartheta}_{\tau}\right) \mathbb{B}: e\left(\dot{u}_{\tau}\right)\right\|_{L^{1}(Q)} & \leqslant \rho_{2}\left\|e\left(\dot{u}_{\tau}\right)\right\|_{\left.L^{2}\left(Q ; \mathbb{R}^{3 \times 3}\right)\right)}^{2}+C_{\rho_{2}}\left\|\Theta\left(\bar{\vartheta}_{\tau}\right)\right\|_{L^{2}(Q)}^{2} \\
& \leqslant \rho_{2}\left\|e\left(\dot{u}_{\tau}\right)\right\|_{\left.L^{2}\left(Q ; \mathbb{R}^{3 \times 3}\right)\right)}^{2}+C_{\rho_{2}}\left(\left\|\bar{\vartheta}_{\tau}\right\|_{L^{2 / \omega}(Q)}^{2 / \omega}+1\right) \\
& \leqslant \rho_{2}\left\|e\left(\dot{u}_{\tau}\right)\right\|_{\left.L^{2}\left(Q ; \mathbb{R}^{3 \times 3}\right)\right)}^{2}+\rho_{3} \int_{0}^{T}\left\|\nabla \bar{\vartheta}_{\tau}\right\|_{L^{r}\left(\Omega ; \mathbb{R}^{3}\right)}^{r} \mathrm{~d} t+C_{\rho_{3}},
\end{aligned}
$$

where the last inequality can be proved, via the Gagliardo-Nirenberg inequality, by developing the same calculations as throughout [39, Formulae (4.39)-(4.43)], and using the restriction on $\omega$ in (4.1b) and the previously proved bound for $\left\|\bar{\vartheta}_{\tau}\right\|_{L^{\infty}\left(0, T ; L^{1}(\Omega)\right)}$. Then, we plug (5.30) into (5.29), and choose $\rho_{2}$ and $\rho_{3}$ in such a way as to absorb the terms $\left\|e\left(\dot{u}_{\tau}\right)\right\|_{L^{2}\left(Q ; \mathbb{R}^{3 \times 3}\right)}^{2}$ and $\left\|\nabla \bar{\vartheta}_{\tau}\right\|_{L^{r}\left(Q ; \mathbb{R}^{3}\right)}^{r}$ into the left-hand side of (5.29). Thus, we conclude estimate (5.25b). We also get an estimate for $\left\|\zeta_{1}\left(\llbracket \bar{u}_{\tau} \rrbracket, \dot{z}_{\tau}\right)\right\|_{L^{1}\left(\Sigma_{\mathrm{C}}\right)}$, which yields (5.25f), since $a_{1}$ is bounded from below, cf. (4.1h). Furthermore, we also obtain a bound for $\nabla \bar{\vartheta}_{\tau}$ in $L^{r}\left(Q ; \mathbb{R}^{3}\right)$. Combining the latter information with the estimate for $\bar{\vartheta}_{\tau}$ in $L^{\infty}\left(0, T ; L^{1}(\Omega)\right)$, we infer $(5.25 \mathrm{~h})$.

Estimate (5.25i) follows from a comparison in (5.15), and the related calculations are a trivial adaptation of the ones in the proof of [35, Lemma 7.7].

Finally, for $(5.25 \mathrm{j})$ we use that $\ddot{u}_{\tau}$ is a measure on $[0, T]$, supported at the jumps of $\dot{u}_{\tau}$, and we estimate $\varrho\left\|\ddot{u}_{\tau}\right\|_{\mathfrak{m}\left(0, T ; W_{\Gamma_{\mathrm{D}}}^{2, \nu}\left(\Omega \backslash \Gamma_{\mathrm{C}} ; \mathbb{R}^{3}\right)^{*}\right)}$ by comparison in (5.12). 


\section{Proof of Theorem 4.2}

In what follows, we develop the proof of the passage to the limit in the time-discrete scheme as $\tau \rightarrow 0$ unifying the cases $\varrho>0$ and $\varrho=0$; we shall take a sequence of time-steps, i.e., we understand $(\tau)$ as countable family of indexes with the accumulation point 0 .

For the reader's convenience, we briefly describe the strategy. After a careful selection of converging subsequences is made in Step 0 below, by passing to the limit as $\tau \rightarrow 0$ in (5.12) we will obtain the weak formulation (3.11b) of the momentum inclusion. Then, we will proceed to proving the semistability condition (3.11d), hence the total energy inequality by lower semicontinuity arguments. By the same tokens we will also obtain the mechanical energy inequality. We will then show that the latter in fact holds as an equality, by combining a chain rule-type argument (cf. (6.19)), with a test of (3.11b) by $\dot{u}$. To perform the latter, it will be essential for $\dot{u}$ to have the regularity (6.28). This motivates the dissipative contribution $\mathbb{G} \nabla e(\dot{u})$ to the hyperstress. Hence, we will exploit the mechanical energy equality to conclude, via a suitable comparison argument, the convergence of the quadratic terms in the right-hand side of (5.15). This will allow us to pass to the limit, and conclude the weak formulation (3.11e) of the enthalpy equation and, ultimately, also the total energy balance (3.11c).

Step 0: Selection of convergent subsequences. First of all, it follows from estimates (5.25b), (5.25c), and (5.25j), from the Banach selection principle, the infinite-dimensional Ascoli and the AubinLions theorems (see, e.g., [45, Thm. 5, Cor. 4]), that there exist a (not relabeled) sequence $\tau \rightarrow 0$ and a limit function $u \in W^{1,2}\left(0, T ; W_{\Gamma_{\mathrm{D}}}^{2,2}\left(\Omega \backslash \Gamma_{\mathrm{C}} ; \mathbb{R}^{3}\right)\right)$ such that the following convergences hold as $\tau \rightarrow 0$ :

$$
\begin{aligned}
& u_{\tau} \rightarrow u \text { in } W^{1,2}\left(0, T ; W_{\Gamma_{\mathrm{D}}}^{2,2}\left(\Omega \backslash \Gamma_{\mathrm{C}} ; \mathbb{R}^{3}\right)\right), \\
& u_{\tau} \rightarrow u \text { in } C\left([0, T] ; W_{\Gamma_{\mathrm{D}}}^{2-\epsilon, 2}\left(\Omega \backslash \Gamma_{\mathrm{C}} ; \mathbb{R}^{3}\right)\right) \quad \forall \epsilon \in(0,2], \\
& \varrho u_{\tau} \stackrel{*}{\rightarrow} \varrho u \text { in } W^{1, \infty}\left(0, T ; L^{2}\left(\Omega ; \mathbb{R}^{3}\right)\right) .
\end{aligned}
$$

Estimate (5.25j) and a generalization of the Aubin-Lions theorem to the case of time derivatives as measures (cf. e.g. [36, Cor. 7.9]) also yield that $\dot{u} \in B V\left([0, T] ; W_{\Gamma_{\mathrm{D}}}^{2, \gamma}\left(\Omega \backslash \Gamma_{\mathrm{C}} ; \mathbb{R}^{3}\right)^{*}\right)$ and that

$$
\varrho \dot{u}_{\tau} \rightarrow \varrho \dot{u} \quad \text { in } L^{2}\left(0, T ; W_{\Gamma_{\mathrm{D}}}^{2-\epsilon, 2}\left(\Omega \backslash \Gamma_{\mathrm{C}} ; \mathbb{R}^{3}\right)\right) \text { for all } \epsilon \in(0,2] .
$$

Moreover, a generalization of Helly's principle (see [2] as well as [29, Thm. 6.1]) implies that $\dot{u}_{\tau}(t) \rightarrow \dot{u}(t)$ in $W_{\Gamma_{\mathrm{D}}}^{2, \gamma}\left(\Omega \backslash \Gamma_{\mathrm{C}} ; \mathbb{R}^{3}\right)^{*}$ for all $t \in[0, T]$. In view of estimate $(5.25 \mathrm{c})$, with an elementary compactness argument we conclude

$$
\varrho \dot{u}_{\tau}(t) \rightarrow \varrho \dot{u}(t) \quad \text { in } L^{2}\left(\Omega ; \mathbb{R}^{3}\right) \text { for all } t \in[0, T]
$$

Next, we observe that

$$
\left\|\bar{u}_{\tau}-u_{\tau}\right\|_{L^{\infty}\left(0, T ; W_{\Gamma_{\mathrm{D}}}^{2,2}\left(\Omega \backslash \Gamma_{\mathrm{C}} ; \mathbb{R}^{3}\right)\right)} \leqslant \tau^{1 / 2}\left\|\dot{u}_{\tau}\right\|_{L^{2}\left(0, T ; W_{\Gamma_{\mathrm{D}}}^{2,2}\left(\Omega \backslash \Gamma_{\mathrm{C}} ; \mathbb{R}^{3}\right)\right)} \leqslant S_{0} \tau^{1 / 2} \rightarrow 0 \quad \text { as } \tau \rightarrow 0 .
$$


Therefore, estimate (5.25a) and (6.1a)-(6.1b) yield for all $\epsilon \in(0,1]$

$$
\begin{aligned}
& \bar{u}_{\tau} \stackrel{*}{\rightarrow} u \text { in } L^{\infty}\left(0, T ; W_{\Gamma_{\mathrm{D}}}^{2,2}\left(\Omega \backslash \Gamma_{\mathrm{C}} ; \mathbb{R}^{3}\right)\right), \\
& \bar{u}_{\tau} \rightarrow u \text { in } L^{\infty}\left(0, T ; W_{\Gamma_{\mathrm{D}}}^{2-\epsilon, 2}\left(\Omega \backslash \Gamma_{\mathrm{C}} ; \mathbb{R}^{3}\right)\right), \\
& \left.\bar{u}_{\tau}(t) \rightarrow u(t) \text { in } W_{\Gamma_{\mathrm{D}}}^{2-\epsilon, 2}\left(\Omega \backslash \Gamma_{\mathrm{C}} ; \mathbb{R}^{3}\right)\right) \text { for all } t \in[0, T] .
\end{aligned}
$$

Taking into account the compact embedding (3.4), from (6.1b) and (6.1h) we deduce respectively

$$
\begin{array}{ll}
\llbracket u_{\tau} \rrbracket \rightarrow \llbracket u \rrbracket & \text { in } C\left(\bar{\Sigma}_{\mathrm{c}} ; \mathbb{R}^{3}\right), \\
\llbracket \bar{u}_{\tau} \rrbracket \rightarrow \llbracket u \rrbracket & \text { in } L^{\infty}\left(\Sigma_{\mathrm{c}} ; \mathbb{R}^{3}\right) .
\end{array}
$$

In fact, the above convergences are also in $C\left([0, T] ; C\left(\bar{\Gamma}_{\mathrm{C}} ; \mathbb{R}^{3}\right)\right)$ and in $L^{\infty}\left(0, T ; L^{\infty}\left(\Gamma_{\mathrm{C}} ; \mathbb{R}^{3}\right)\right)$, respectively. Convergences $(6.1 \mathrm{~g}-\mathrm{i}, \mathrm{k})$ hold for $\underline{u}_{\tau}$, too. Also note that, in view of $(5.25 \mathrm{~d})$, we have

$$
\left.\begin{array}{l}
\tau\left\|\left|e\left(\bar{u}_{\tau}\right)\right|^{\gamma-2} e\left(\bar{u}_{\tau}\right)\right\|_{L^{\gamma /(\gamma-1)}\left(Q ; \mathbb{R}^{3 \times 3}\right)} \leqslant S_{0} \tau^{1 / \gamma} \rightarrow 0 \text { and } \\
\tau\left\|\left|\nabla e\left(\bar{u}_{\tau}\right)\right|^{\gamma-2} \nabla e\left(\bar{u}_{\tau}\right)\right\|_{L^{\gamma /(\gamma-1)}\left(Q ; \mathbb{R}^{3 \times 3 \times 3}\right)} \leqslant S_{0} \tau^{1 / \gamma} \rightarrow 0
\end{array}\right\} \quad \text { as } \tau \rightarrow 0 .
$$

Estimates (5.25e) and (5.25f), and the very same compactness arguments as in [35, Sec. 8] (based on [29, Thm. 6.1, Prop. 6.2]), also guarantee that there exists a function $z \in L^{\infty}\left(\Sigma_{\mathrm{C}}\right) \cap B V([0, T] ; Z)$ (where $\mathcal{Z}$ is any reflexive space such that $L^{1}\left(\Gamma_{\mathrm{C}}\right) \subset \mathcal{Z}$ with a continuous embedding), such that, possibly along a subsequence,

$$
\bar{z}_{\tau} \stackrel{*}{\rightarrow} z \text { in } L^{\infty}\left(\Sigma_{\mathrm{C}}\right), \quad \bar{z}_{\tau}(t) \stackrel{*}{\rightarrow} z(t) \quad \text { in } L^{\infty}\left(\Gamma_{\mathrm{C}}\right) \text { for all } t \in[0, T] .
$$

We now prove that

$$
\operatorname{Var}_{\mathcal{R}}(u, z ;[s, t]) \leqslant \liminf _{\tau \rightarrow 0} \int_{s}^{t} \int_{\Gamma_{\mathrm{C}}} \zeta_{1}\left(\llbracket \bar{u}_{\tau} \rrbracket, \dot{z}_{\tau}\right) \mathrm{d} S \mathrm{~d} r \quad \text { for all } 0 \leqslant s \leqslant t \leqslant T .
$$

Indeed,

$$
\begin{aligned}
\left.\int_{S}^{t} \int_{\Gamma_{\mathrm{C}}} \zeta_{1}\left(\llbracket \bar{u}_{\tau}\right], \dot{z}_{\tau}\right) \mathrm{d} S \mathrm{~d} r=\int_{s}^{t} \int_{\Gamma_{\mathrm{C}}} a_{1}\left(\left[\bar{u}_{\tau} \rrbracket\right)\left|\dot{z}_{\tau}\right| \mathrm{d} S \mathrm{~d} r\right. \\
\left.\quad=\int_{s}^{t} \int_{\Gamma_{\mathrm{C}}} a_{1}\left(\llbracket u_{\tau}\right]\right)\left|\dot{z}_{\tau}\right| \mathrm{d} S \mathrm{~d} r+\int_{s}^{t} \int_{\Gamma_{\mathrm{C}}}\left(a_{1}\left(\left[\bar{u}_{\tau} \rrbracket\right)-a_{1}\left(\llbracket u_{\tau}\right]\right)\right)\left|\dot{z}_{\tau}\right| \mathrm{d} S \mathrm{~d} r .
\end{aligned}
$$

Now, from $(6.1 \mathrm{j}, \mathrm{k})$ and the continuity of $a_{1}$ it follows

$$
\left.a_{1}\left(\llbracket \bar{u}_{\tau} \rrbracket\right)-a_{1}\left(\llbracket u_{\tau}\right]\right) \rightarrow 0 \quad \text { in } L^{\infty}\left(0, T ; C\left(\bar{\Gamma}_{\mathrm{C}}\right)\right) .
$$

Since $\left(\dot{z}_{\tau}\right)_{\tau>0}$ is bounded in $L^{1}\left(\Sigma_{\mathrm{C}}\right)$, we then conclude that the second term on the right-hand side of (6.4) tends to zero as $\tau \rightarrow 0$. To pass to the limit in the first term, we use (6.1j) and again the continuity of $a_{1}$. Since $\left|\dot{z}_{\tau}\right| \rightarrow|\dot{z}|$ weakly* in the sense of measures on $\bar{\Sigma}_{\mathrm{C}}$, we conclude that

$$
\left.a_{1}\left(\llbracket u_{\tau}\right]\right)\left|\dot{z}_{\tau}\right| \rightarrow a_{1}(\llbracket u \rrbracket)|\dot{z}| \quad \text { weakly* in the sense of measures on } \bar{\Sigma}_{\mathrm{c}},
$$


where $|\dot{z}|$ denotes the variation of the measure $\dot{z}$; in fact, here simply $|\dot{z}|=-\dot{z}$, since $\dot{z} \leqslant 0$. Then, (6.3) follows. Taking into account that $a_{1}$ is bounded from below by (4.1h), (6.3) and the definition (3.14) of $\operatorname{Var}_{\mathcal{R}}$ imply $z \in B V\left([0, T] ; L^{1}\left(\Gamma_{\mathrm{C}}\right)\right)$.

With the same compactness tools as in the above lines, we conclude from estimates $(5.25 \mathrm{~g})$, (5.25h), and (5.25i) that there exists $\vartheta \in L^{r}\left(0, T ; W^{1, r}\left(\Omega \backslash \Gamma_{\mathrm{C}}\right)\right) \cap B V\left([0, T] ; W^{1, r^{\prime}}\left(\Omega \backslash \Gamma_{\mathrm{C}}\right)^{*}\right)$ s.t.

$$
\begin{aligned}
& \bar{\vartheta}_{\tau} \rightarrow \vartheta \text { and } \vartheta_{\tau} \rightarrow \vartheta \text { in } L^{r}\left(0, T ; W^{1, r}\left(\Omega \backslash \Gamma_{\mathrm{C}}\right)\right) \text { for } 1 \leqslant r<\frac{5}{4}, \\
& \bar{\vartheta}_{\tau}, \vartheta_{\tau} \rightarrow \vartheta \text { in } L^{r}\left(0, T ; W^{1-\epsilon, r}\left(\Omega \backslash \Gamma_{\mathrm{C}}\right)\right) \text { for all } \epsilon \in(0,1] .
\end{aligned}
$$

The latter convergence yields that $\bar{\vartheta}_{\tau}, \vartheta_{\tau} \rightarrow \vartheta$ in $L^{r}\left(0, T ; L^{15 / 7-\epsilon}(\Omega)\right)$ for all $\epsilon \in(0,8 / 7]$. Taking into account estimate $(5.25 \mathrm{~g})$ in $L^{\infty}\left(0, T ; L^{1}(\Omega)\right)$ and arguing by interpolation, we conclude

$$
\bar{\vartheta}_{\tau}, \vartheta_{\tau} \rightarrow \vartheta \quad \text { in } L^{5 / 3-\epsilon}(Q) \text { for all } \epsilon \in\left(0, \frac{2}{3}\right]
$$

Notice that, under condition (4.7) on $\theta_{0},(5.7)$ and (6.7c) imply the strict positivity of $\vartheta$. Moreover, Helly's selection principle and the a priori bound for $\left(\bar{\vartheta}_{\tau}\right)_{\tau}$ in $L^{\infty}\left(0, T ; L^{1}(\Omega)\right)$ yield

$$
\vartheta_{\tau}(t) \stackrel{*}{\rightarrow} \vartheta(t) \quad \text { in } m(\bar{\Omega}) \text { for all } t \in[0, T] .
$$

Combining (6.7c) with (4.2), it is immediate to deduce

$$
\Theta\left(\bar{\vartheta}_{\tau}\right) \rightarrow \Theta(\vartheta) \quad \text { in } L^{2}(Q)
$$

Furthermore, it follows from (6.7b), the trace theorem $\vartheta \mapsto \llbracket \vartheta \rrbracket: W^{1-\epsilon, r}\left(\Omega \backslash \Gamma_{\mathrm{C}}\right) \rightarrow L^{10 / 7-\epsilon}\left(\Gamma_{\mathrm{C}}\right)$ for all $\epsilon \in(0,3 / 7]$, and (4.2), that

$$
\llbracket \Theta\left(\bar{\vartheta}_{\tau}\right) \rrbracket \rightarrow \llbracket \Theta(\vartheta) \rrbracket \quad \text { in } L^{r \omega}\left(0, T ; L^{\omega(10 / 7-\epsilon)}\left(\Gamma_{\mathrm{C}}\right)\right) \forall \epsilon \in\left(0, \frac{5}{7}\right] .
$$

Exploiting (6.1i), (6.2), and (6.1k), which in particular yields $\lim _{\tau \rightarrow 0} \int_{\Gamma_{\mathrm{C}}} \bar{z}_{\tau}(t) \alpha_{0}\left(\llbracket \bar{u}_{\tau}(t) \rrbracket\right) \mathrm{d} S=$ $\int_{\Gamma_{\mathrm{C}}} z(t) \alpha_{0}(\llbracket u(t) \rrbracket) \mathrm{d} S$ for all $t \in[0, T]$, we conclude by lower semicontinuity arguments that

$$
\Phi(u(t), z(t)) \leqslant \liminf _{\tau \rightarrow 0} \Phi_{\tau}\left(\bar{u}_{\tau}(t), \bar{z}_{\tau}(t)\right) \quad \text { for all } t \in[0, T] .
$$

Step 1: Passage to the limit in the momentum equation. At first, we take the limit as $\tau \rightarrow 0$ of the discrete momentum equation (5.12) with smooth test functions $v \in C^{\infty}\left(Q ; \mathbb{R}^{3}\right)$, fulfilling $\llbracket v \rrbracket \succeq 0$ on $\Sigma_{\mathrm{C}}$. We approximate them with discrete approximations $\left\{v_{\tau}^{k}\right\}$ such that $\llbracket v_{\tau}^{k} \rrbracket \succeq 0$ on $\Sigma_{\mathrm{C}}$ and the related piecewise constant and linear interpolants fulfill, as $\tau \rightarrow 0$,

$$
\left.\begin{array}{ll}
v_{\tau} \rightarrow v & \text { in } W^{1,1}\left(0, T ; L^{2}\left(\Omega ; \mathbb{R}^{3}\right)\right), \\
\bar{v}_{\tau} \rightarrow v & \text { in } L^{2}\left(0, T ; W^{2,2}\left(\Omega \backslash \Gamma_{\mathrm{C}} ; \mathbb{R}^{3}\right)\right), \\
\left\|\nabla e\left(\bar{v}_{\tau}\right)\right\|_{L^{\nu}\left(Q ; \mathbb{R}^{3 \times 3 \times 3}\right)} \leqslant C . &
\end{array}\right\}
$$

In order to pass to the limit in the first integral term on the left-hand side of (5.12), we use (6.1a), (6.1b), and (6.8), combined with (6.11). The regularizing $\gamma$-terms in (5.12) vanish in the limit due to the last of (6.11). Notice that (6.1k) and the continuity of $\alpha_{0}^{\prime}$ (cf. (4.1f)) imply $\alpha_{0}^{\prime}\left(\llbracket \bar{u}_{\tau} \rrbracket\right) \rightarrow \alpha_{0}^{\prime}(\llbracket u \rrbracket)$ in $L^{\infty}\left(\Sigma_{\mathrm{C}}\right)$. Therefore, taking (6.2) into account, we deduce that $\underline{z}_{\tau} \alpha_{0}^{\prime}\left(\llbracket \bar{u}_{\tau} \rrbracket\right) \stackrel{*}{\rightarrow} z \alpha_{0}^{\prime}(\llbracket u \rrbracket)$ in $L^{\infty}\left(\Sigma_{\mathrm{C}}\right)$. 
This convergence and (6.11) allow us to pass to the limit in the second integral term on the lefthand side of (5.12). To take the limit of the third and fourth terms (in the case $\varrho>0$ ), we use (6.1d) and (6.1e) as well as the first of (6.11). The latter allows us also to take the limit of the first term on the right-hand side of (5.12). The convergence of the other two integrals ensues from (5.8), (6.1a), and (6.11). Thus, we have proved that the triple $(u, z, \vartheta)$ fulfills equation (3.11b) with smooth test functions. With a density argument, we conclude (3.11b) with test functions $v \in$ $L^{2}\left(0, T ; W_{\Gamma_{\mathrm{D}}}^{2,2}\left(\Omega \backslash \Gamma_{\mathrm{C}} ; \mathbb{R}^{3}\right)\right) \cap W^{1,1}\left(0, T ; L^{2}\left(\Omega ; \mathbb{R}^{3}\right)\right)$. For later convenience, let us observe that, in the case $K(x)$ is a linear subspace for almost all $x \in \Gamma_{\mathrm{C}}$ (cf. (4.5)), taking test functions $\tilde{v}=u+\lambda v$ with $v$ any admissible test function satisfying $\llbracket v \rrbracket \succeq 0$ on $\Sigma_{\mathrm{C}}$ and $\lambda$ an arbitrary real number, we obtain (3.11b) in the form

$$
\begin{aligned}
\int_{\Omega} \varrho \dot{u}(T) \cdot v(T) \mathrm{d} x+\int_{Q}(\mathbb{D} e(\dot{u})+\mathbb{C} e(u)-\mathbb{B} \Theta(\vartheta)): e(v) & \\
+(\mathbb{H} \nabla e(u)+\mathbb{G} \nabla e(\dot{u})) & \left.: \nabla e(v)-\varrho \dot{u} \cdot \dot{v} \mathrm{~d} x \mathrm{~d} t+\int_{\Sigma_{\mathrm{C}}} z \alpha_{0}^{\prime}(\llbracket u \rrbracket) \cdot \llbracket v\right] \mathrm{d} S \mathrm{~d} t \\
& =\int_{\Omega} \varrho \dot{u}_{0} \cdot v(0) \mathrm{d} x+\int_{Q} F \cdot v \mathrm{~d} x \mathrm{~d} t-\int_{\Sigma_{\mathrm{N}}} f \cdot v \mathrm{~d} S \mathrm{~d} t
\end{aligned}
$$

for any $v \in L^{2}\left(0, T ; W_{K}^{2,2}\left(\Omega \backslash \Gamma_{\mathrm{c}} ; \mathbb{R}^{3}\right)\right) \cap W^{1,1}\left(0, T ; L^{2}\left(\Omega ; \mathbb{R}^{3}\right)\right)$.

Step 2: Passage to the limit in the semistability condition. We consider a subset $\eta \subset(0, T)$ of full measure such that for all $t \in \eta$ the approximate stability condition (5.14) holds for the (countably many) considered $\tau$ 's. Then we fix $t \in \eta$ and $\tilde{z} \in L^{\infty}\left(\Gamma_{\mathrm{C}}\right)$. We may suppose without loss of generality that $R(u(t), \tilde{z}-z(t))<+\infty$, hence

$$
\tilde{z}(x) \leqslant z(t, x) \quad \text { for a.a. } x \in \Gamma_{\mathrm{C}} .
$$

Then, we construct the following recovery sequence

$$
\tilde{z}_{\tau}(t, x):= \begin{cases}\bar{z}_{\tau}(t, x) \frac{\tilde{z}(x)}{z(t, x)} & \text { where } z(t, x)>0, \\ 0 & \text { where } z(t, x)=0 .\end{cases}
$$

Now, using (6.13) and (6.2) one immediately sees that

$$
\tilde{z}_{\tau}(t, \cdot) \leqslant \bar{z}_{\tau}(t, \cdot) \quad \text { a.e. in } \Gamma_{\mathrm{C}}, \quad \tilde{z}_{\tau}(t) \stackrel{*}{\rightarrow} \tilde{z} \text { in } L^{\infty}\left(\Gamma_{\mathrm{c}}\right) .
$$

Plugging $\tilde{z}_{\tau}$ in (5.14) and using (6.1k), (6.2), and (6.15), we find

$$
\begin{aligned}
0 & \leqslant \lim _{\tau \rightarrow 0}\left(\Phi_{\tau}\left(\bar{u}_{\tau}(t), \tilde{z}_{\tau}(t)\right)+R\left(\bar{u}_{\tau}(t), \tilde{z}_{\tau}(t)-\bar{z}_{\tau}(t)\right)-\Phi_{\tau}\left(\bar{u}_{\tau}(t), \bar{z}_{\tau}(t)\right)\right) \\
& =\lim _{\tau \rightarrow 0} \int_{\Gamma_{\mathrm{C}}}\left(\alpha_{0}\left(\llbracket \bar{u}_{\tau}(t) \rrbracket\right)-a_{1}\left(\llbracket \bar{u}_{\tau}(t) \rrbracket\right)\right)\left(\tilde{z}_{\tau}(t)-\bar{z}_{\tau}(t)\right) \mathrm{d} S \\
& =\int_{\Gamma_{\mathrm{C}}}\left(\alpha_{0}(\llbracket u(t) \rrbracket)-a_{1}(\llbracket u(t) \rrbracket)\right)(\tilde{z}(t)-\bar{z}(t)) \mathrm{d} S \\
& =\Phi(u(t), \tilde{z}(t))+R(u(t), \tilde{z}(t)-z(t))-\Phi(u(t), z(t)) .
\end{aligned}
$$


Step 3: Passage to the limit in the mechanical and total energy inequalities. Using (6.1a), (6.1e), (6.3), and (6.10), we pass to the limit on the left-hand side of the discrete mechanical energy inequality (5.16) by weak lower semicontinuity. To take the limit of the right-hand side, we employ (5.2) (which in particular yields $\Phi_{\tau}\left(u_{0, \tau}, z_{0}\right) \rightarrow \Phi\left(u_{0}, z_{0}\right)$ ), the weak convergence (6.1a) and the strong convergence (6.8), which give

$$
\Theta\left(\bar{\vartheta}_{\tau}\right) \mathbb{B}: e\left(\dot{u}_{\tau}\right) \rightarrow \Theta(\vartheta) \mathbb{B}: e(\dot{u}) \quad \text { weakly in } L^{1}(Q) .
$$

We pass to the limit in the two remaining terms by $(5.8 \mathrm{a})-(5.8 \mathrm{~b})$. Hence, the triple $(u, z, \vartheta)$ complies for all $t \in[0, T]$ with

$$
\begin{aligned}
T_{\text {kin }}(\dot{u}(t)) & +\Phi(u(t), z(t))+\int_{0}^{t} \int_{\Omega} \mathbb{D} e(\dot{u}(s)): e(\dot{u}(s))+\mathbb{G} \nabla e(\dot{u}(s)) \vdots \nabla e(\dot{u}(s)) \mathrm{d} x \mathrm{~d} s \\
& +\operatorname{Var}_{\mathcal{R}}(u, z ;[0, t]) \leqslant T_{\text {kin }}\left(\dot{u}_{0}\right)+\Phi\left(u_{0}, z_{0}\right) \\
& +\int_{0}^{t}\left(\int_{\Omega} \Theta(\vartheta(s)) \mathbb{B}: e(\dot{u}(s)) \mathrm{d} x+\int_{\Omega} F(s) \cdot \dot{u}(s) \mathrm{d} x+\int_{\Gamma_{\mathrm{N}}} f(s) \cdot \dot{u}(s) \mathrm{d} S\right) \mathrm{d} s .
\end{aligned}
$$

By the very same lower semicontinuity arguments (also using (6.7d) and (5.9)), we also pass to the limit in the discrete total energy inequality (5.13).

Step 4: Mechanical energy equality. First of all, we observe that the following chain rule-type inequality holds for all $t \in[0, T]$

$$
\begin{aligned}
& \Phi(u(t), z(t))-\Phi\left(u_{0}, z_{0}\right)+\operatorname{Var}_{\mathbb{R}}(u, z ;[0, t]) \geqslant \int_{0}^{t}\langle\lambda, \dot{u}\rangle \mathrm{d} s \\
& \quad \text { for any } \lambda \in L^{2}\left(0, T ; W^{2,2}\left(\Omega \backslash \Gamma_{\mathrm{C}} ; \mathbb{R}^{3}\right)^{*}\right) \text { with } \lambda(t) \in \partial_{u} \Phi(u(t), z(t)) \text { for a.a. } t \in(0, T),
\end{aligned}
$$

where $\partial_{u} \Phi: W^{2,2}\left(\Omega \backslash \Gamma_{\mathrm{C}} ; \mathbb{R}^{3}\right) \rightrightarrows W^{2,2}\left(\Omega \backslash \Gamma_{\mathrm{C}} ; \mathbb{R}^{3}\right)^{*}$ denotes the subdifferential w.r.t. $u$ of the functional $\Phi: W^{2,2}\left(\Omega \backslash \Gamma_{\mathrm{C}} ; \mathbb{R}^{3}\right) \times L^{\infty}\left(\Gamma_{\mathrm{C}}\right) \rightarrow \mathbb{R}$ defined in (3.8). Easy calculations show that the operator $\partial_{u} \Phi$ is given by

$$
\begin{aligned}
& \lambda \in \partial_{u} \Phi(u, z) \text { if and only if } \exists \ell \in \partial \ell_{K}(u) \forall v \in W^{2,2}\left(\Omega \backslash \Gamma_{\mathrm{C}} ; \mathbb{R}^{3}\right): \\
& \langle\lambda, v\rangle=\int_{\Omega} \mathbb{C} e(u): e(v)+\mathbb{H} \nabla e(u) \vdots \nabla e(v) \mathrm{d} x+\int_{\Gamma_{\mathrm{C}}} z \alpha_{0}^{\prime}(\llbracket u \rrbracket) \cdot \llbracket v \rrbracket \mathrm{d} S+\langle\ell, v\rangle,
\end{aligned}
$$

where, for notational convenience, we have introduced the functional $\ell_{K}: W^{2,2}\left(\Omega \backslash \Gamma_{\mathrm{C}} ; \mathbb{R}^{3}\right) \rightarrow$ $[0,+\infty]$ defined by $\ell_{K}(u)=I_{K}(\llbracket u \rrbracket)$ (cf. (2.9)), and its subdifferential $\partial_{\ell_{K}}: W^{2,2}\left(\Omega \backslash \Gamma_{\mathrm{C}} ; \mathbb{R}^{3}\right) \rightrightarrows$ $W^{2,2}\left(\Omega \backslash \Gamma_{\mathrm{c}} ; \mathbb{R}^{3}\right)^{*}$. In order to prove (6.19) for a fixed selection $\lambda(t) \in \partial_{u} \Phi(u(t), z(t))$, we exploit a technique, combining Riemann sums and the already proved semistability condition (3.11d), which is well-known in the analysis of rate-independent systems and dates back to [9]. The main difficulty here is to adapt such a trick to the case of a Stieltjes integral (cf. (6.25) below), and to do so we will mimic the argument in the proof of [41, Prop. 3]. For any $n>0$, we take a suitable partition $0=t_{0}^{n}<t_{1}^{n}<\ldots<t_{N_{n}}^{n}=T$ with $\max _{i=1, \ldots, N_{n}}\left(t_{i}^{n}-t_{i-1}^{n}\right) \leqslant 1 / n$, in such a way that the functions $Q_{n}:[0, T] \rightarrow L^{\infty}\left(\Gamma_{\mathrm{C}}\right)$ given by $Q_{n}(t):=a_{1}\left(\llbracket u\left(t_{i-1}^{n}\right) \rrbracket\right)$ for $t \in\left(t_{i-1}^{n}, t_{i}^{n}\right]$ fulfill

$$
Q_{n} \rightarrow a_{1}([u]) \text { in } L^{\infty}\left(\Sigma_{\mathrm{C}}\right) \text { as } n \rightarrow \infty .
$$


The existence of such partitions follows from the fact that $u:[0, T] \rightarrow W^{2,2}\left(\Omega \backslash \Gamma_{\mathrm{C}} ; \mathbb{R}^{3}\right)$ is continuous, since $u \in W^{1,2}\left(0, T ; W^{2,2}\left(\Omega \backslash \Gamma_{\mathrm{c}} ; \mathbb{R}^{3}\right)\right)$. Thus it is also uniformly continuous, and so is the mapping $\llbracket u \rrbracket:[0, T] \rightarrow L^{\infty}\left(\Gamma_{\mathrm{C}}\right)$. Then, we use that uniformly continuous mappings admit uniform approximation by piecewise constant interpolants. In fact, (6.21) holds for all partitions of $[0, T]$ whose fineness tends to 0 , therefore we can choose our partition in such a way that the semistability (3.11d) holds at all points $\left\{t_{i}^{n}: i=0, \ldots, N_{n}-1, n \in \mathbb{N}\right\}$. Hence, we write (3.11d) at $t_{i-1}^{n}$ tested by $\tilde{z}=z\left(t_{i}^{n}\right)$, thus obtaining

$$
\begin{aligned}
& \Phi\left(u\left(t_{i-1}^{n}\right), z\left(t_{i-1}^{n}\right)\right) \leqslant \Phi\left(u\left(t_{i-1}^{n}\right), z\left(t_{i}^{n}\right)\right)+\mathbb{R}\left(u\left(t_{i-1}^{n}\right), z\left(t_{i}^{n}\right)-z\left(t_{i-1}^{n}\right)\right) \\
& =\Phi\left(u\left(t_{i}^{n}\right), z\left(t_{i}^{n}\right)\right)+\int_{\Gamma_{\mathrm{C}}} a_{1}\left(\llbracket u\left(t_{i-1}^{n}\right) \rrbracket\right)\left|z\left(t_{i}^{n}\right)-z\left(t_{i-1}^{n}\right)\right| \mathrm{d} S-\int_{t_{i-1}^{n}}^{t_{i}^{n}}\left\langle\lambda_{n}(s), \dot{u}(s)\right\rangle \mathrm{d} s
\end{aligned}
$$

for any selection $\lambda_{n}(t) \in \partial_{u} \Phi\left(u(t), z\left(t_{i}^{n}\right)\right)$ for a.a. $t \in\left(t_{i-1}^{n}, t_{i}^{n}\right], i=1, \ldots, N_{n}$, where we have also used the chain rule for the convex functional $u \mapsto \Phi\left(u, z\left(t_{i}^{n}\right)\right)$, cf. [52, Prop. XI.4.11]. In particular, taking into account formula (6.20) for $\partial_{u} \Phi$, we choose

$$
\begin{aligned}
& \lambda_{n}(t)=\lambda(t)-\rho_{n}(t) \text { with } \rho_{n}(t) \in W^{2,2}\left(\Omega \backslash \Gamma_{\mathrm{C}} ; \mathbb{R}^{3}\right)^{*} \text { given by } \\
&\left\langle\rho_{n}(t), v\right\rangle:=\int_{\Gamma_{\mathrm{C}}}\left(z(t)-z\left(t_{i}^{n}\right)\right) \alpha_{0}^{\prime}(\llbracket u(t) \rrbracket) \cdot \llbracket v \rrbracket \mathrm{d} S .
\end{aligned}
$$

Summing for $i=1, \ldots, N_{n}$, we obtain

$$
\Phi(u(T), z(T))-\Phi\left(u_{0}, z_{0}\right)+\sum_{i=1}^{N_{n}} \int_{t_{i-1}^{n}}^{t_{i}^{n}} \int_{\bar{\Gamma}_{\mathrm{C}}} \mathrm{Q}_{n}|\dot{z}|(\mathrm{d} S \mathrm{~d} t) \geqslant \sum_{i=1}^{N_{n}} \int_{t_{i-1}^{n}}^{t_{i}^{n}}\left\langle\lambda_{n}(s), \dot{u}(s)\right\rangle \mathrm{d} s .
$$

Now, reproducing the calculations throughout [39, Formulae (4.70)-(4.74)], it can be shown that

$$
\liminf _{n \rightarrow \infty} \sum_{i=1}^{N_{n}} \int_{t_{i-1}^{n}}^{t_{i}^{n}}\left\langle\lambda_{n}(s), \dot{u}(s)\right\rangle \mathrm{d} s \geqslant \int_{0}^{t}\langle\lambda(s), \dot{u}(s)\rangle \mathrm{d} s .
$$

On the other hand, it follows from (6.21) that

$$
\left.\lim _{n \rightarrow \infty} \sum_{i=1}^{N_{n}} \int_{t_{i-1}^{n}}^{t_{i}^{n}} \int_{\bar{\Gamma}_{\mathrm{C}}} Q_{n}|\dot{z}|(\mathrm{d} S \mathrm{~d} t)=\int_{\bar{\Sigma}_{\mathrm{C}}} a_{1}(\llbracket u]\right) \dot{z}(\mathrm{~d} S, d t)=\operatorname{Var}_{\mathbb{R}}(u, z ;[0, T]) .
$$

Indeed, $\dot{z} \in C\left(\bar{\Sigma}_{\mathrm{C}}\right)^{*}$ can be extended to $L^{\infty}\left(\Sigma_{\mathrm{C}}\right)^{*}$ by the Hahn-Banach principle, and then tested by $Q_{n}-a_{1}(\llbracket u \rrbracket) \in L^{\infty}\left(\Sigma_{\mathrm{C}}\right)$ which converges to zero by (6.21). Combining (6.23)-(6.25), we obtain (6.19).

In order to make (6.19) more explicit, we may observe that

$$
\left.\int_{0}^{t}\langle\ell, \dot{u}\rangle \mathrm{d} s=\ell_{K}(u(t))-\ell_{K}(u(0))=I_{K}(\llbracket u(t) \rrbracket)-I_{K}(\llbracket u(0)]\right)=0
$$

for all $\ell \in L^{2}\left(0, T ; W^{2,2}\left(\Omega \backslash \Gamma_{\mathrm{C}} ; \mathbb{R}^{3}\right)^{*}\right)$ such that $\ell(s) \in \partial \ell_{K}(u(s))$ for a.a. $s \in(0, T)$, (6.26) 
by the chain rule for the convex functional $\ell_{K}$ (cf. again [52, Prop. XI.4.11]), and by (3.7a) and (3.11a). Therefore, in view of (6.19)-(6.26), we conclude the following inequality for all $t \in[0, T]$

$$
\begin{aligned}
& \Phi(u(t), z(t))-\Phi\left(u_{0}, z_{0}\right)+\operatorname{Var}_{\mathbb{R}}(u, z ;[0, t]) \\
& \left.\geqslant \int_{0}^{t}\left(\int_{\Omega} \mathbb{C} e(u): e(\dot{u})+\mathbb{H} \nabla e(u): \nabla e(\dot{u}) \mathrm{d} x+\int_{\Gamma_{\mathrm{C}}} z \alpha_{0}^{\prime}(\llbracket u]\right) \cdot[\dot{u}] \mathrm{d} S\right) \mathrm{d} s .
\end{aligned}
$$

In order to develop the test of (3.11b) by $\dot{u}$, we need to distinguish the quasistatic case $\varrho=0$ and the dynamical case $\varrho>0$.

Case $\varrho>0$. First of all, let us observe that, under (4.5), the qualification $v \in$ $W^{1,2}\left(0, T ; L^{2}\left(\Omega ; \mathbb{R}^{d}\right)\right)$ for the test functions in (3.11b) might be relaxed to

$$
v \in L^{2}\left(0, T ; W_{K}^{2,2}\left(\Omega \backslash \Gamma_{\mathrm{C}} ; \mathbb{R}^{3}\right)\right) \cap W^{1,2}\left(0, T ; W_{K}^{2,2}\left(\Omega \backslash \Gamma_{\mathrm{C}} ; \mathbb{R}^{d}\right)^{*}\right),
$$

cf. notation (3.5). Indeed, thanks to (3.11a) and to the linearity of $K(x)$ for almost all $x \in \Gamma_{\mathrm{C}}$, the function $u$ fulfilling (3.11b) is such that $\dot{u} \in L^{2}\left(0, T ; W_{K}^{2,2}\left(\Omega \backslash \Gamma_{\mathrm{C}} ; \mathbb{R}^{d}\right)\right)$. Note that (6.28) is sufficient to give meaning to the term $\int_{Q} \dot{u} \cdot \dot{v} \mathrm{~d} x \mathrm{~d} t$, because the spaces $L^{2}\left(0, T ; W_{K}^{2,2}\left(\Omega \backslash \Gamma_{\mathrm{C}} ; \mathbb{R}^{d}\right)^{*}\right)$ and $L^{2}\left(0, T ; W_{K}^{2,2}\left(\Omega \backslash \Gamma_{\mathrm{C}} ; \mathbb{R}^{d}\right)\right)$ are in duality.

Now, a comparison in (6.12) yields that $\ddot{u} \in L^{2}\left(0, T ; W_{K}^{2,2}\left(\Omega \backslash \Gamma_{\mathrm{C}} ; \mathbb{R}^{3}\right)^{*}\right)$. Therefore, (4.6) ensues, and $\dot{u}$ is an admissible test function for the momentum balance inclusion (3.11b), since it fulfills (6.28). Then, upon proceeding with such a test we conclude for all $t \in[0, T]$ that

$$
\begin{aligned}
\frac{\varrho}{2} \int_{\Omega}|\dot{u}(t)|^{2} \mathrm{~d} x & +\int_{0}^{t} \int_{\Omega} \mathbb{D} e(\dot{u}): e(\dot{u})+\mathbb{G} \nabla e(\dot{u}): \nabla e(\dot{u}) \mathrm{d} x \mathrm{~d} s \\
& \left.\left.+\int_{0}^{t} \int_{\Omega} \mathbb{C} e(u): e(\dot{u})+\mathbb{H} \nabla e(u) \vdots \nabla e(\dot{u}) \mathrm{d} x \mathrm{~d} s+\int_{0}^{t} \int_{\Gamma_{\mathrm{C}}} z \alpha_{0}^{\prime}(\llbracket u]\right) \cdot \llbracket \dot{u}\right] \mathrm{d} S \mathrm{~d} s \\
= & \frac{\varrho}{2} \int_{\Omega}\left|\dot{u}_{0}\right|^{2} \mathrm{~d} x+\int_{0}^{t}\left(\int_{\Omega} \Theta(\vartheta) \mathbb{B}: e(\dot{u}) \mathrm{d} x+\int_{\Omega} F \cdot \dot{u} \mathrm{~d} x+\int_{\Gamma_{\mathrm{N}}} f \cdot \dot{u} \mathrm{~d} S\right) \mathrm{d} s .
\end{aligned}
$$

Combining (6.29) with (6.27), we get the converse of inequality (6.18), hence the desired mechanical energy equality (3.13) ensues.

Case $\varrho=0$ : A comparison in (3.11b) with $\varrho=0$ shows that the functional

$$
\begin{aligned}
\ell: v \mapsto \int_{Q}(\mathbb{D} e(\dot{u})+\mathbb{C} e(u)-\mathbb{B} \Theta(\vartheta)): e(v)+(\mathbb{G} \nabla e(\dot{u})+\mathbb{H} \nabla e(u)): \nabla e(v) \mathrm{d} x \mathrm{~d} t \\
+\int_{\Sigma_{\mathrm{C}}} z \alpha_{0}^{\prime}(\llbracket u \rrbracket) \cdot \llbracket v \rrbracket \mathrm{d} S \mathrm{~d} t-\int_{Q} F \cdot v \mathrm{~d} x \mathrm{~d} t-\int_{\Sigma_{\mathrm{N}}} f \cdot v \mathrm{~d} S \mathrm{~d} t
\end{aligned}
$$

is in $L^{2}\left(0, T ; W^{2,2}\left(\Omega \backslash \Gamma_{\mathrm{C}} ; \mathbb{R}^{3}\right)^{*}\right)$, and fulfills

$$
\int_{0}^{T} I_{K}(\llbracket v \rrbracket) \mathrm{d} t \geqslant \int_{0}^{T} I_{K}(\llbracket u \rrbracket) \mathrm{d} t+\int_{0}^{T}\langle\ell, v-u\rangle \mathrm{d} t .
$$


Hence, $\ell(t) \in \partial \ell_{K}(u(t))$ for almost all $t \in(0, T)$. Thus, (6.26) yields $\int_{0}^{t}\langle\ell, \dot{u}\rangle \mathrm{d} s=0$ for all $t \in[0, T]$, which is just relation (6.29) with $\varrho=0$. Again, we combine the latter with (6.27), and conclude the mechanical energy equality (3.13).

Step 5: Passage to the limit in the enthalpy equation. First of all, we observe the following chain of inequalities for all $t \in[0, T]$ :

$$
\begin{aligned}
& \operatorname{Var}_{\mathbb{R}}(u, z ;[0, t])+\int_{0}^{t} \int_{\Omega} \mathbb{D} e(\dot{u}): e(\dot{u})+\mathbb{G} \nabla e(\dot{u}): \nabla e(\dot{u}) \mathrm{d} x \mathrm{~d} s \\
& \leqslant \liminf _{\tau \rightarrow 0} \int_{0}^{t} \int_{\Gamma_{\mathrm{C}}} \zeta_{1}\left(\llbracket \bar{u}_{\tau} \rrbracket, \dot{z}_{\tau}\right) \mathrm{d} S \mathrm{~d} s+\int_{0}^{t} \int_{\Omega} \mathbb{D} e\left(\dot{u}_{\tau}\right): e\left(\dot{u}_{\tau}\right)+\mathbb{G} \nabla e\left(\dot{u}_{\tau}\right): \nabla e\left(\dot{u}_{\tau}\right) \mathrm{d} x \mathrm{~d} s \\
& \leqslant \limsup _{\tau \rightarrow 0} T_{\operatorname{kin}}\left(\dot{u}_{0, \tau}\right)+\Phi_{\tau}\left(u_{0, \tau}, z_{0}\right)-T_{\mathrm{kin}}\left(\dot{u}_{\tau}(t)\right)-\Phi_{\tau}\left(\bar{u}_{\tau}(t), \bar{z}_{\tau}(t)\right) \\
& \quad+\int_{0}^{t}\left(\int_{\Omega} \Theta\left(\bar{\vartheta}_{\tau}\right) \mathbb{B}: e\left(\dot{u}_{\tau}\right)+\int_{\Omega} \bar{F}_{\tau} \cdot \dot{u}_{\tau} \mathrm{d} x+\int_{\Gamma_{\mathrm{N}}} \bar{f}_{\tau} \cdot \dot{u}_{\tau} \mathrm{d} S\right) \mathrm{d} s \\
& \leqslant T_{\operatorname{kin}}\left(\dot{u}_{0}\right)+\Phi\left(u_{0}, z_{0}\right)-T_{\operatorname{kin}}(\dot{u}(t))-\Phi(u(t), z(t)) \\
& \quad+\int_{0}^{t}\left(\int_{\Omega} \Theta(\vartheta) \mathbb{B}: e(\dot{u})+F \cdot \dot{u} \mathrm{~d} x+\int_{\Gamma_{\mathrm{N}}} f \cdot \dot{u} \mathrm{~d} S\right) \mathrm{d} s \\
& =\operatorname{Diss}_{\mathbb{R}}(u, z ;[0, t])+\int_{0}^{t} \int_{\Omega} \mathbb{D} e(\dot{u}): e(\dot{u})+\mathbb{G} \nabla e(\dot{u}): \nabla e(\dot{u}) \mathrm{d} x \mathrm{~d} s .
\end{aligned}
$$

Indeed, the first inequality ensues from (6.1a) and (6.3), the second one from the discrete mechanical energy inequality (5.16), the third one from (5.2), (6.1e), (6.10), (6.17), and from (5.8a)-(5.8b), cf. also Step 3. Finally, the last equality ensues from the mechanical energy equality (3.13) proved in Step 4. Thus, all of the above inequalities turn out to hold as equalities. By a standard liminf/limsup argument, we find in particular

$\mathbb{D} e\left(\dot{u}_{\tau}\right): e\left(\dot{u}_{\tau}\right) \rightarrow \mathbb{D} e(\dot{u}): e(\dot{u}) \quad$ and $\quad \mathbb{G} \nabla e\left(\dot{u}_{\tau}\right): \nabla e\left(\dot{u}_{\tau}\right) \rightarrow \mathbb{G} \nabla e(\dot{u}): \nabla e(\dot{u})$ strongly in $L^{1}(Q)$.

Combining these convergences with (6.17) we pass to the limit in the first term on the right-hand side of the discrete enthalpy equation (5.15). To take the limit of the second right-hand-side term, we observe that

$$
\begin{aligned}
\lim _{\tau \rightarrow 0} \int_{\Sigma_{\mathrm{C}}} a_{1}\left(\llbracket \bar{u}_{\tau} \rrbracket\right) \dot{z}_{\tau} v \mathrm{~d} S \mathrm{~d} t & =\lim _{\tau \rightarrow 0} \int_{\Sigma_{\mathrm{C}}}\left(a_{1}\left(\llbracket \bar{u}_{\tau} \rrbracket\right)-a_{1}\left(\llbracket u_{\tau} \rrbracket\right)\right) \dot{z}_{\tau} v \mathrm{~d} S \mathrm{~d} t \\
& \left.+\lim _{\tau \rightarrow 0} \int_{\Sigma_{\mathrm{C}}} a_{1}\left(\llbracket u_{\tau}\right]\right) \dot{z}_{\tau} v \mathrm{~d} S \mathrm{~d} t=0+\int_{\Sigma_{\mathrm{C}}} a_{1}(\llbracket u \rrbracket) v \dot{z}(\mathrm{~d} S \mathrm{~d} t)
\end{aligned}
$$

for any $v \in C\left(\bar{\Sigma}_{\mathrm{C}}\right)$, and in particular for $v=\frac{\left.w\right|_{\Gamma_{\mathrm{C}}} ^{+}+\left.w\right|_{\Gamma_{\mathrm{C}}}}{2}$; here we used respectively (6.5), (6.6), and (5.25f). Then, we pass to the limit in the left-hand side of (5.15) by exploiting (6.1b), (6.7a), (6.7b), (6.7d), (6.9), as well as properties (4.1c) for $\mathcal{K}$ and (4.1e) for $\eta$, and by arguing in the very same way as in the proof of [35, Thm. 5.1], to which we refer for all details.

In the end, employing (5.9), we take the limit of the last term on the right-hand side of (5.15), thus finding that the triple $(u, z, \vartheta)$ fulfils the weak formulation (3.11e) of the enthalpy equation.

Step 6: Total energy identity. We test the weak formulation (3.11e) of the enthalpy equation by 1 and add it to the mechanical energy equality. This gives the total energy balance (3.11c). 
Acknowledgments. This research was initiated during a visit of R.R. at the Charles University in Prague, whose hospitality is gratefully acknowledged, with the support of the "Nečas center for mathematical modeling" LC 06052 (MŠMT ČR), and partially of the PRIN 2008 project "Optimal mass transportation, geometric and functional inequalities and applications". T.R. acknowledges the hospitality of the University of Brescia supported from Istituto Nazionale di Alta Matematica (GNAMPA project), as well as partial support from the grants A 100750802 (GA AV ČR), 201/09/0917 and 201/10/0357 (GA ČR), together with the institutional support RVO: 61388998

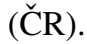

\section{REFERENCES}

1. BANKS-SILls, L. AND ASKENAZI, D., A note on fracture criteria for interface fracture. Intl. J. Fracture 103 (2000), 177-188.

2. Barbu, V. and Precupanu, T., Convexity and Optimization in Banach Spaces. D. Reidel Pub. Co. 2nd edition (1986). Zbl0594.49001 MR0860772

3. BocCARdo, L. AND GallouËt, T., Non-linear elliptic and parabolic equations involving measure data. J. Funct. Anal. 87 (1989), 149-169. Zb10707.35060 MR1025884

4. Bonetti, E., Bonfanti, G. And Rossi, R., Global existence for a contact problem with adhesion. Math. Methods Appl. Sci. 31 (2008), 1029-1064. Zbl1145. 35301 MR2419088

5. Bonetti, E., Bonfanti, G. And Rossi, R., Well-posedness and long-time behaviour for a model of contact with adhesion. Indiana Univ. Math. J. 56 (2007), 2787-2820. Zbl1145. 35027 MR2375702

6. Bonetti, E., Bonfanti, G. And Rossi, R., Thermal effects in adhesive contact: modelling and analysis. Nonlinearity 22 (2009), 2697-2731. Zbl1185.35122 MR2550692

7. Bonetti, E., Bonfanti, G. And Rossi, R., Long-time behaviour of a thermomechanical model for adhesive contact. Discrete Contin. Dyn. Syst. Ser. S. 4 (2011), 273-309. Zb11217. 35025 MR2746376

8. Bonetti, E., Bonfanti, G. And Rossi, R., Analysis of a unilateral contact problem taking into account adhesion and friction. J. Differential Equations 253 (2012), 438-462. Zbl pre06052893Mr2921201

9. Dal Maso, G., Francfort, G. And ToAder, R., Quasistatic crack growth in nonlinear elasticity. Arch. Rational Mech. Anal. 176 (2005), 165-225. Zbl1064.74150 MR2186036

10. ECK, C., JARUŠEK, J. AND KRBEC, M., Unilateral contact problems. Chapman \& Hall/CRC (2005). Zb11079.74003 MR2128865

11. Evans, A.G., Rühle, M., Dalgleish, B.J. and Charalambides, P.G., The fracture energy of bimaterial interfaces. Metallurgical Transactions A 21A (1990), 2419-2429.

12. Feireisl, E. AND MÁleK, J., On the Navier-Stokes equations with temperature-dependent transport coefficients. Diff. Equations Nonlin. Mech. (2006), 14 pp. (electronic), Art.ID 90616. Zbl1133. 35419 MR2233755

13. Frémond, M., Equilibre des structures qui adhèrent à leur support. Comptes Rendus Acad. Sci. Paris 295 (1982), 913-916. Zbl0551. 73096 MR0695554

14. Frémond, M., Adhèrence des solides. Journal de Méchanique Théorique et Appliquée 6 (1987), 383407. Zb10645.73046 MR0912217

15. Fried, E. And GuRTin, M.E., Tractions, balances, and boundary conditions for nonsimple materials with application to liquid flow at small-lenght scales. Arch. Rational Mech. Anal. 182 (2006), 513-554. Zb11104.74006 MR2276499

16. Griffith, A.A., The phenomena of rupture and flow in solids. Philos. Trans. Royal Soc. London Ser. A. Math. Phys. Eng. Sci. 221 (1921), 163-198.

17. Hutchinson, J.W. And Wu, T.Y., Mixed mode cracking in layered materials. In: Advances in Appl. Mech. (Eds. J.W. Hutchinson, T.Y.Wu), Acad. Press, New York, (1992), pp. 63-191. Zb10790.73056 
18. KJelstrup, S. ANd BedeAuX, D., Non-equilibrium Thermodynamics of Heterogeneous Systems, World Scientific, (2008). Zbl1142.82002 MR2401047

19. KneEs, D., Rossi, R. AND ZANini, C., A vanishing viscosity approach to a rate-independent damage model. Math. Models Methods Appl. Sci. 23 (2013), 565-616. Zblpre06162054

20. Kočvara, M., Mielke, A. And RoubíčEK, T., A rate-independent approach to the delamination problem. Mathematics and Mechanics of Solids 11 (2006), 423-447. Zbl1133. 74038 MR2245202

21. LARSEn, C.J., ORTner, C. AND SÜLI, E., Existence of solution to a regularized model of dynamic fracture. Math. Models Meth. Appl. Sci. 20 (2010), 1021-1048. Zblpre05781032 MR2673410

22. Liechti, K.M. AND ChaI, Y.S., Asymmetric shielding in interfacial fracture under in-plane shear, $J$. Appl. Mech. 59 (1992), 295-304.

23. Mantič, V., Discussion on the reference lenght and mode mixity for a bimaterial interface. J. Engr. Mater. Technology 130 (2008), 045501-1-2.

24. Mielke, A., Evolution in rate-independent systems. In: Handbook of Differential Equations, Evolutionary Equations, 2 (Eds.: Dafermos, C.M., Feireisl, E.), Elsevier(2005), 461-559. Zb11120. 47062 MR2182832

25. Mielke, A., Thermomechanical modeling of energy-reaction-diffusion systems, including bulkinterface interactions. Discrete Contin. Dyn. Syst. Ser. S6 (2013), 479-499. Zblpre06144728 MR2997555

26. Mielke, A. , Roubíček, T. And Thomas, M., From damage to delamination in nonlinearly elastic materials at small strain. J. Elasticity 109 (2012), 235-273. Zbl1253.74088 MR2971959

27. Mielke, A. ANd Theil, F., A mathematical model for rate-independent phase transformations with hysteresis. In: Models of Cont. Mech. in Analysis and Engr. (Eds.: ALBER, H.-D. BALEAN, R.M., AND FARWIG, R.), Shaker-Verlag (1999), 117-129.

28. Mielke, A., Theil, F. And Levitas, V., A variational formulation of rate-independent phase transformations using an extremum principle. Arch. Rat. Mech. Anal. 162 (2002), 137-177. Zbl1012. 74054 MR1897379

29. Mielke, A. And Theil, F., On rate-independent hysteresis models. Nonlin. Diff. Equations Appl. 11 (2004), 151-189. (Accepted July 2001). Zbl1061. 35182 MR2210284

30. Petrov, A. And Schatzman, M., Mathematical results on existence for viscoelastodynamic problems with unilateral constraints. SIAM J. Math. Anal. 40 (2009), 1882-1904. Zbl1179. 35198 MR2471904

31. Podio-Guidugli, P. And Vianello, M., Hypertractions and hyperstresses convey the same mechanical information. Cont. Mech. Thermodynam. 22 (2010), 163-176. Zblpre05774285 MR2602440

32. Point, N., Unilateral contact with adherence. Math. Methods Appl. Sci. 10 (1988), 367-381. Zblo656. 73052 MR0958479

33. Raous, M., CAngÉmi, L. And Cocou, M., A consistent model coupling adhesion, friction, and unilateral contact. Comput. Methods Appl. Mech. Eng. 177 (1999), 383-399. MR1710458

34. RitTEL, D., Thermomechanical aspects of dynamic crack initiation. Intl. J. Fracture 99 (1999), 199-209.

35. Rossi, R. AND RoubíčEK, T., Thermodynamics and analysis of rate-independent adhesive contact at small strains. Nonlin. Anal. 74 (2011), 3159-3190. Zb11217. 35108 MR2793554

36. RoubíčEK, T. , Nonlinear Partial Differential Equations with Applications. Birkhäuser (2005) (2nd. ed., 2013).

37. RoubíčEK, T., Thermo-visco-elasticity at small strains with $L^{1}$-data. Quarterly Appl. Math. 67 (2009), 47-71. Zbl1160.74011 MR2495071

38. RoubíčEk, T., Rate independent processes in viscous solids at small strains. Math. Methods Appl. Sci. 32 (2009), 825-862. Zbl1239.35158 MR2507935

39. RoubíčEK, T., Thermodynamics of rate independent processes in viscous solids at small strains. SIAM J. Math. Anal. 40 (2010), 256-297. Zbl1213. 35279 MR2596554 
40. RoubíčEK, T., Nonlinearly coupled thermo-visco-elasticity. Nonlin. Diff. Eq.Appl., in print. DOI10. 1007/s00030-012-0207-9

41. RoubíčEK, T., Thermodynamics of perfect plasticity. Discrete Contin. Dyn. Syst. Ser. S. 6 (2013), 193214. MR2983475

42. RoubíčEK, T., KRuŽíK, M. AND Zeman, J., Delamination and adhesive contact models and their mathematical analysis and numerical treatment. Chapter 13 in: Math. Methods and Models in Composites (Ed. Mantič, V.), Imperial College Press, 2013, 349-400. In print, ISBN 978-1-84816-784-1.

43. RoubíčEK, T., MAntič, V. And PANagiotopoulos, C.G., Quasistatic mixed-mode delamination model. Discrete Contin. Dyn. Syst. Ser. S. (2012), to appear.

44. Roubíček, T., Scardia, L. And Zanini, C., Quasistatic delamination problem. Cont. Mech. Thermodynam. 21 (2009), 223-235. Zb11179.74130 MR2529453

45. Simon, J., Compact sets in the space $L^{p}(0, T ; B)$. Ann. Mat. Pura Appl., 146 (1987), 65-96. Zblo629. 46031 MR0916688

46. Sofonea, M., HAN, W. AND Shillor, M., Analysis and Approximation of Contact Problems with Adhesion or Damage. Chapman \& Hall/CRC (2006). Zbl1089.74004 MR2183435

47. Swadener, J.G., Liechti, K.M. And DeLozanne, A.L., The intrinsic toughness and adhesion mechanism of a glass/epoxy interface. J. Mech. Phys. Solids 47 (1999), 223-258. Zb10947.74501

48. Távara, L. , Mantič, V., Graciani, E., Canas, J. And París, J.F., Analysis of a crack in a thin adhesive layer between orthotropic materials: An application to composite interlaminar fracture toughness test. CMES 58 (2010), 247-270. Zbl1231.74086

49. TÁvara, L., MANTIČ, V., GRACiAni, E. AND PARís, F., BEM analysis of crack onset and propagation along fiber-matrix interface under transverse tension using a linear elastic-brittle interface model. Engr. Analysis with Boundary Elements 35 (2011), 207-222. MR2740346

50. Toupin, R.A., Elastic materials with couple stresses. Arch. Rat. Mech. Anal. 11 (1962), 385-414. Zb10112.16805 MR0144512

51. TVeergard, W. And Hutchinson, J.V., The influence of plasticity on mixed mode interface toughness. Intl. J. Physics of Solids 41 (1993), 1119-1135. Zb10775.73219

52. VISInTIN, A., Models of Phase Transitions. Birkhäuser (1996). MR1423808 